\title{
Precipitation-Chemistry Data at Selected Sites in Northwestern Colorado, 1980-94
}

By Anthony J. Ranalli

U.S. GEOLOGICAL SURVEY

Open-File Report 97-112

Prepared in cooperation with

DELTA, MESA, AND RIO BLANCO COUNTIES, COLORADO

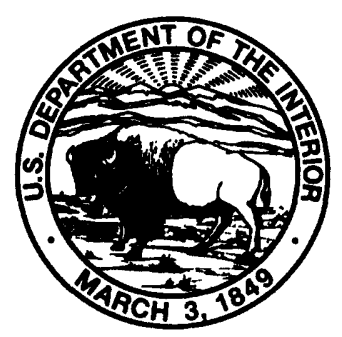




\title{
U.S. DEPARTMENT OF THE INTERIOR \\ BRUCE BABBITT, Secretary
}

\author{
U.S. GEOLOGICAL SURVEY
}

Gordon P. Eaton, Director

The use of firm, trade, and brand names in this report is for identification purposes only and does not constitute endorsement by the U.S. Geological Survey.

For additional information write to:

District Chief

U.S. Geological Survey

Box 25046, Mail Stop 415

Denver Federal Center

Denver, CO 80225-0046
Copies of this report can be purchased from:

U.S. Geological Survey

Branch of Information Services

Box 25286

Denver, CO 8022-0286 


\section{CONTENTS}

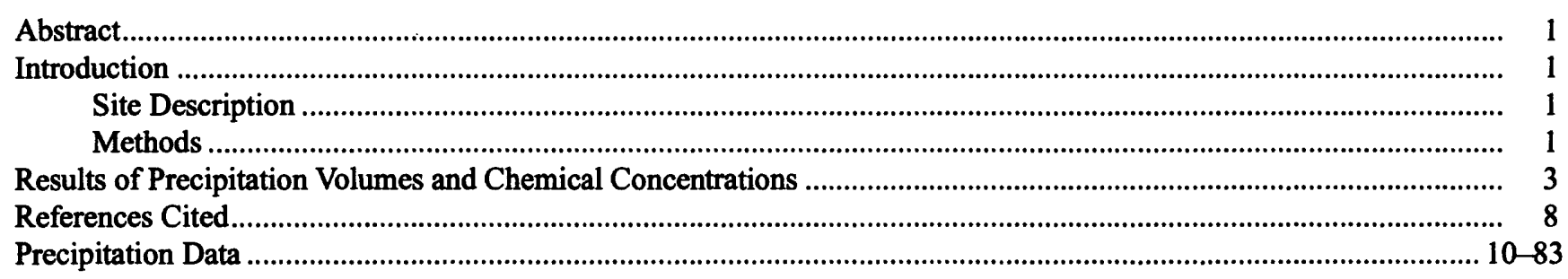

\section{FIGURES}

1. Map showing location of selected precipitation-monitoring sites in northwestern Colorado

2-10. Box plots showing:

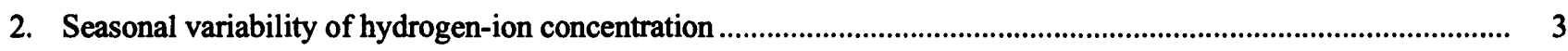

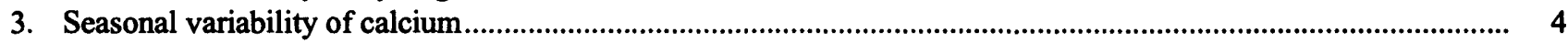

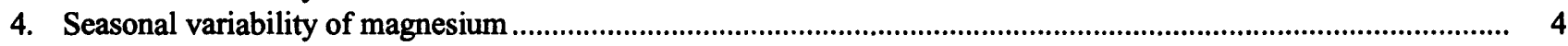

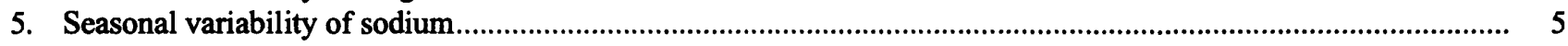

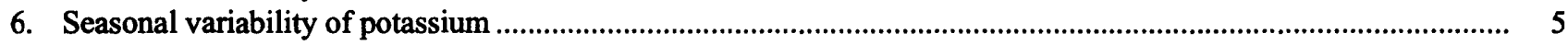

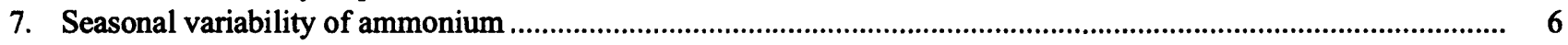

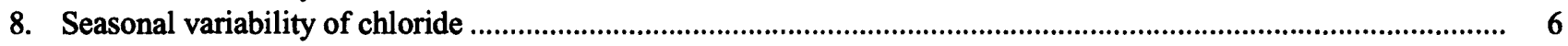

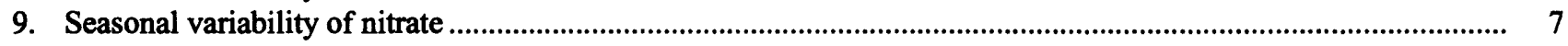

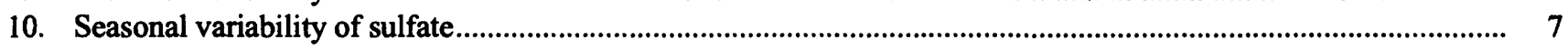

\section{TABLES}

1. Precipitation volume, specific conductance, concentrations of dissolved ions, and percent charge balance for each sampling interval at Douglas Pass, 1980-91

2. Summary statistics of specific conductance and concentrations of dissolved ions at Douglas Pass, 1980-91 .......... 17

3. Precipitation volume, specific conductance, concentrations of dissolved ions, and percent charge balance for each sampling interval at Grand Mesa, 1981-91.

4. Summary statistics of specific conductance and concentrations of dissolved ions at Grand Mesa, 1981-91............ 25

5. Precipitation volume, specific conductance, concentrations of dissolved ions, and percent charge balance for each sampling interval at Marvine Ranch, 1981-94.

6. Summary statistics of specific conductance and concentrations of dissolved ions at Marvine Ranch, 1981-94 ....... 46

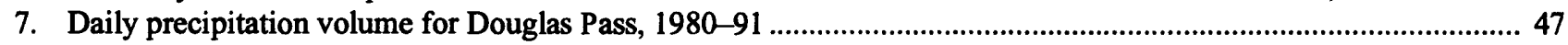

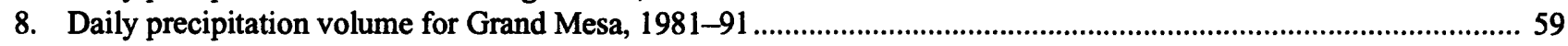

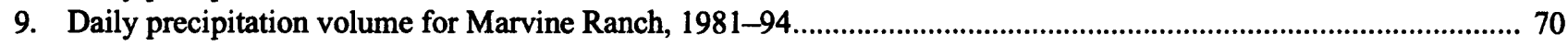




\title{
Precipitation-Chemistry Data at Selected Sites in Northwestern Colorado, 1980-94
}

\author{
By Anthony J. Ranalli
}

\begin{abstract}
The chemical content of precipitation was monitored during the period $1980-94$ at three sites at altitudes above 2,400 meters near the Piceance Basin in northwestern Colorado. Daily precipitation volumes, specific conductance, $\mathrm{pH}$ (in this report $\mathrm{pH}$ is expressed as hydrogen-ion concentration), concentrations of major cations and anions, calculated charge balance between cations and anions, and summary statistics of chemical concentrations are tabulated. Sampling sites are plotted on a map of the area. Seasonal variabilities of major cation and anion concentrations are presented graphically.
\end{abstract}

\section{INTRODUCTION}

Knowledge of the chemistry of precipitation is needed in the preparation of permit requests for new sources of emissions to the atmosphere and for review of these requests. One area in which data may be needed is northwestern Colorado. Energy resources, such as oil shale and coal, are upwind from the federally protected Flat Tops Wilderness Area, which is sensitive to acid rain (Turk and Adams, 1983). The lack of precipitation-chemistry data in northwestern Colorado partially was addressed by establishment of a network of monitoring sites as part of a study done by the U.S. Geological Survey, in cooperation with Delta, Mesa, and Rio Blanco Counties, and assisted by the Rocky Mountain Oil and Gas Association. The network is located near the oil shale reserves of the Piceance Basin. Data collected from these sites provide a baseline for ambient atmospheric deposition that could be compared to any future measurements. The data also are suitable for making estimates of natural variability in precipitation chemistry. This report presents data collected at the monitoring sites from 1980 to 1994 and includes site location, sampling and laboratory analytical methods, precipitation volume and summary statistics, and seasonal variability of major chemical constituents.

\section{Site Description}

The three precipitation-monitoring sites (fig. 1) are located at Douglas Pass at an altitude of 2,710 meters in a treeless area of sage; at Grand Mesa, at an altitude of 3,121 meters in a clearing; and at Marvine Ranch, at an altitude of 2,414 meters in a clearing.

\section{Methods}

Precipitation volume and chemistry have been monitored at Douglas Pass since November 1980, at Grand Mesa since August 1981, and at Marvine Ranch since April 1981. Data collection was discontinued at Douglas Pass and Grand Mesa in October 1991 but is ongoing at Marvine Ranch. The sampling equipment and analytical methods are the same at each site. The sampling intervals, however, varied at each site. The sampling equipment consisted of a Belfort weighingbucket rain gage for the measurement of precipitation volume and an Aerochem Metrics Model 301 wet/dry collector for the monitoring of wet deposition. The samples collected at each site are a combination of single storms and two or more storms collected into a single sample. Unfiltered precipitation samples were analyzed for specific conductance, $\mathrm{pH}$, dissolved calcium, magnesium, sodium, potassium, ammonium, chloride, nitrate, and sulfate by the U.S. Geological Survey National Water-Quality Laboratory in Arvada, 

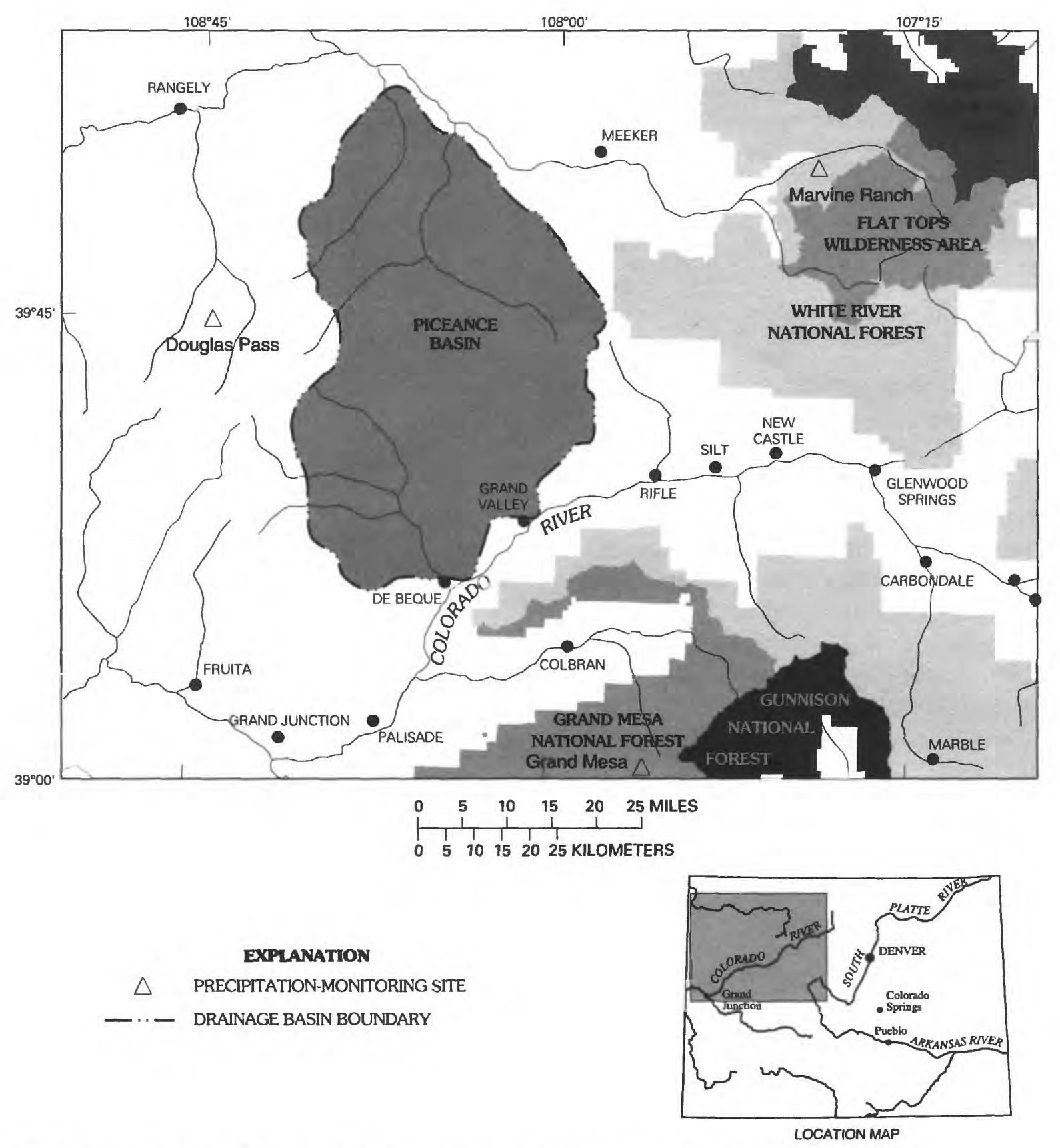

Figure 1. Location of selected precipitation-monitoring sites in northwestern Colorado. 
Colorado, using methods described in Fishman and Friedman (1989). After May 1991, samples were filtered through 0.45-micrometer filters. Aliquots for cations were preserved with concentrated nitric acid, whereas aliquots for ammonium were preserved with mercuric chloride. The chemical data were checked using cation:anion balances and comparisons of measured and calculated specific conductance.

Alkalinity was not measured because $\mathrm{pH}$ typically was low enough to make alkalinity minimal. The buckets used to collect precipitation were cleaned by scrubbing with a nylon brush and rinsing with deionized water. Bias and precision data for the chemical analysis done at the U.S. Geological Survey National Water-Quality Laboratory are published yearly in U.S. Geological Survey Water-Resources Investigations reports (Peart and Sutphin, 1987; Lucey and Peart, 1988; Lucey and Peart, 1989a, 1989b, 1989c; Maloney and others 1992, 1993, 1994).

\section{RESULTS OF PRECIPITATION VOLUMES AND CHEMICAL CONCENTRATIONS}

Specific conductance, precipitation chemical concentrations, and calculated charge balance are presented in tables 1,3 , and 5. Daily precipitation volumes are reported in tables 7-9. Summary statistics (tables 2, 4, and 6) of specific conductance and chemical concentrations (means, medians, standard deviations, and minimum and maximum values) follow each data table. Seasonal variations of precipitation chemistry at each site are shown in the box plots in figures 2-10.

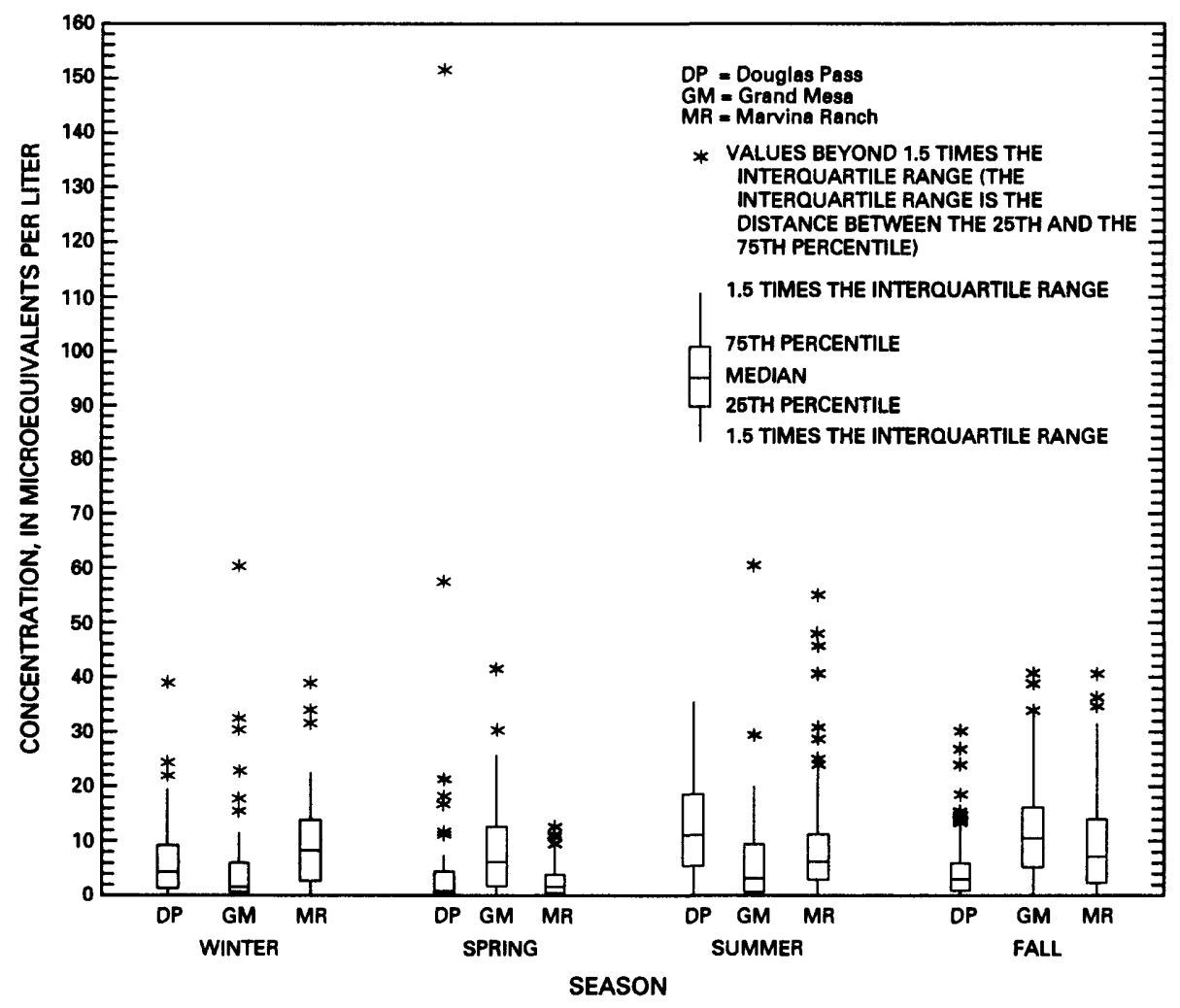

Figure 2. Seasonal variability of hydrogen-ion concentration. 


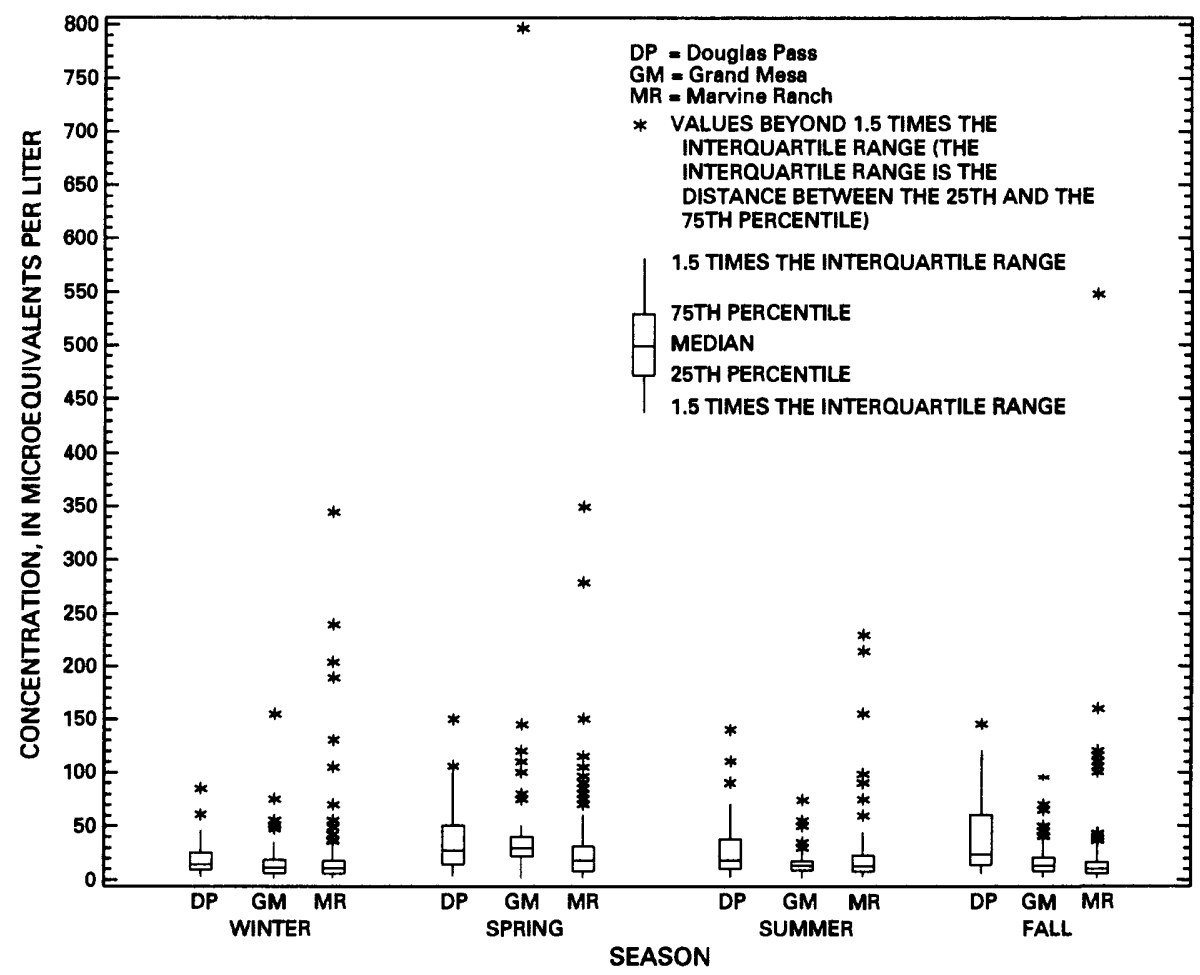

Figure 3. Seasonal variability of calcium.

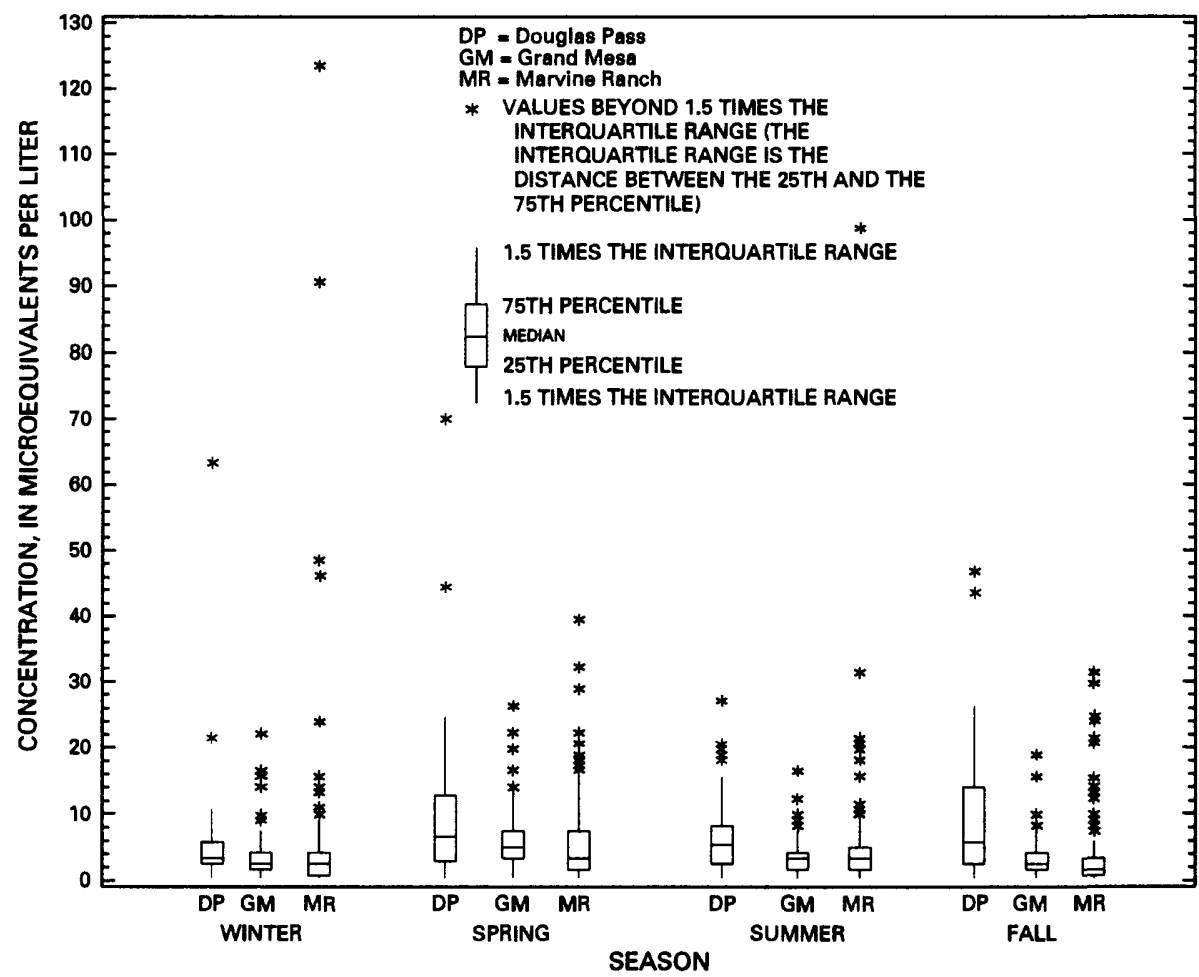

Figure 4. Seasonal variability of magnesium. 


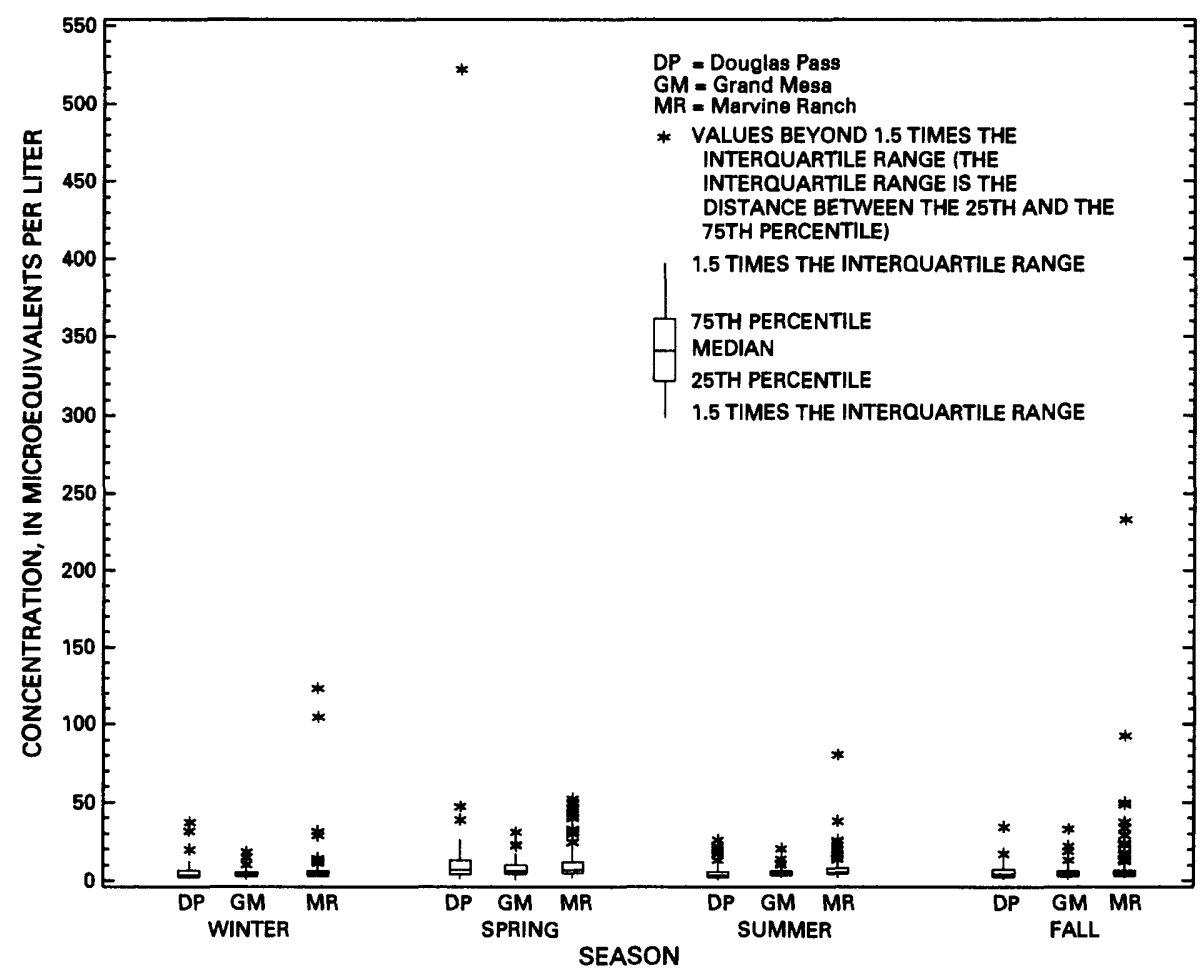

Figure 5. Seasonal variability of sodium.

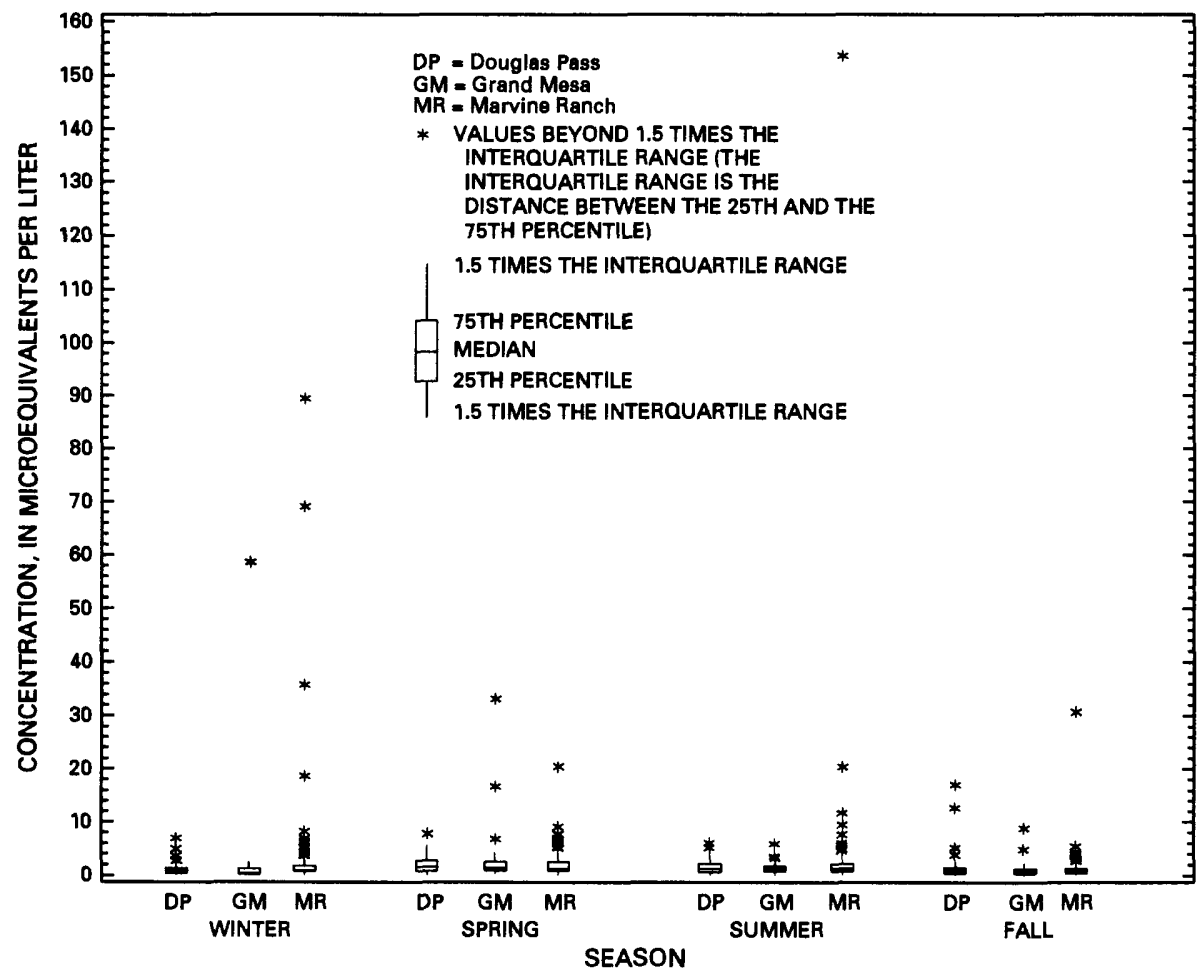

Figure 6. Seasonal variability of potassium. 


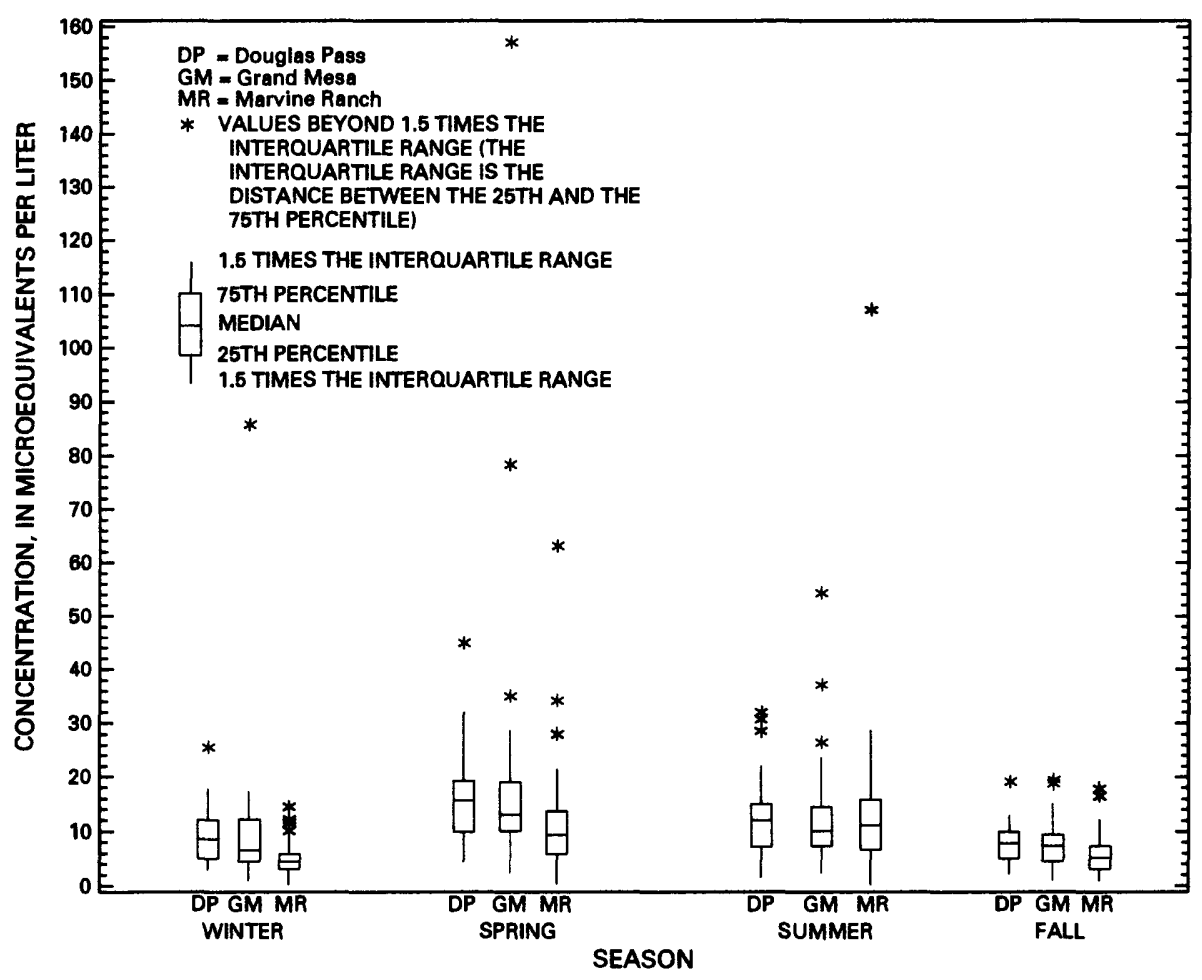

Figure 7. Seasonal variability of ammonium.

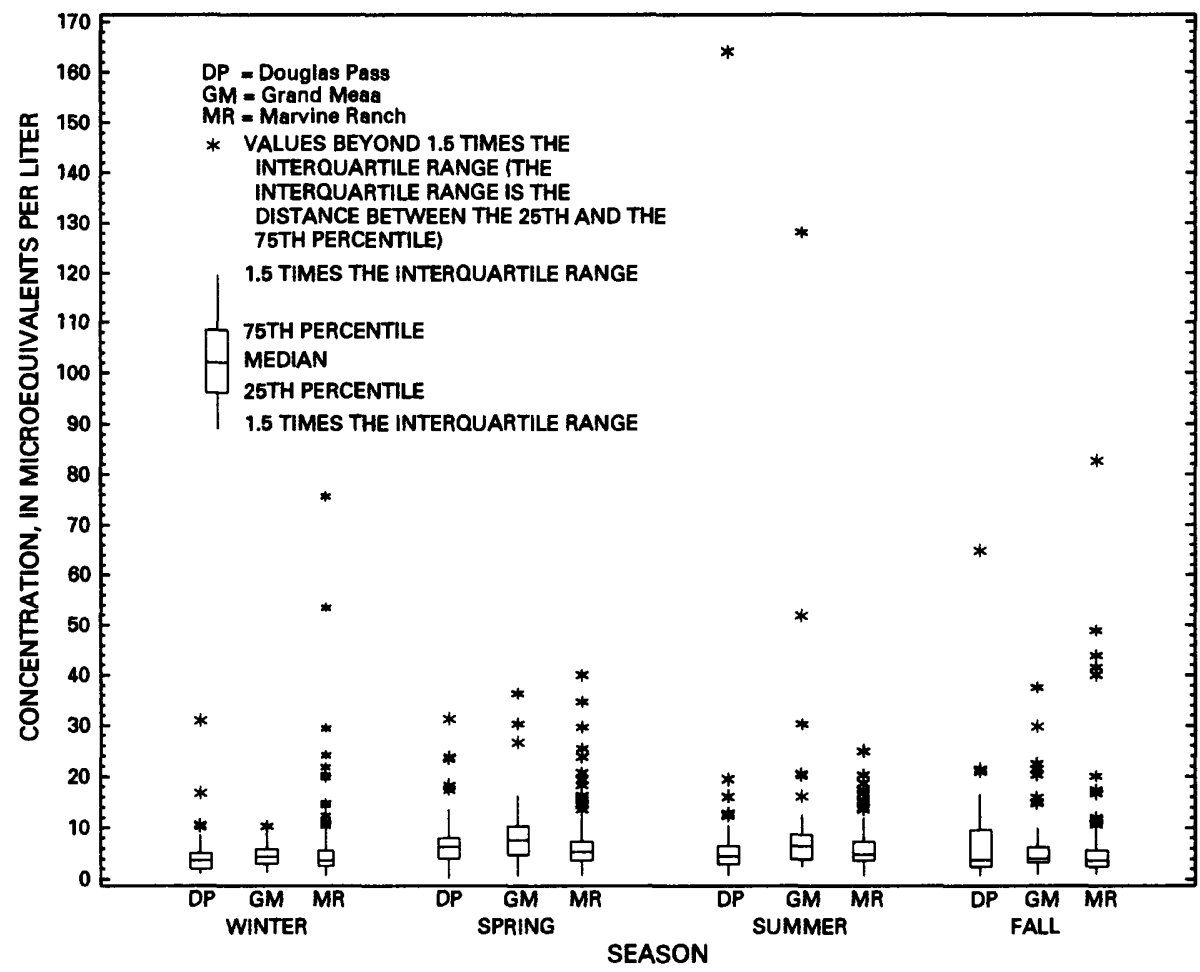

Figure 8. Seasonal variability of chloride. 


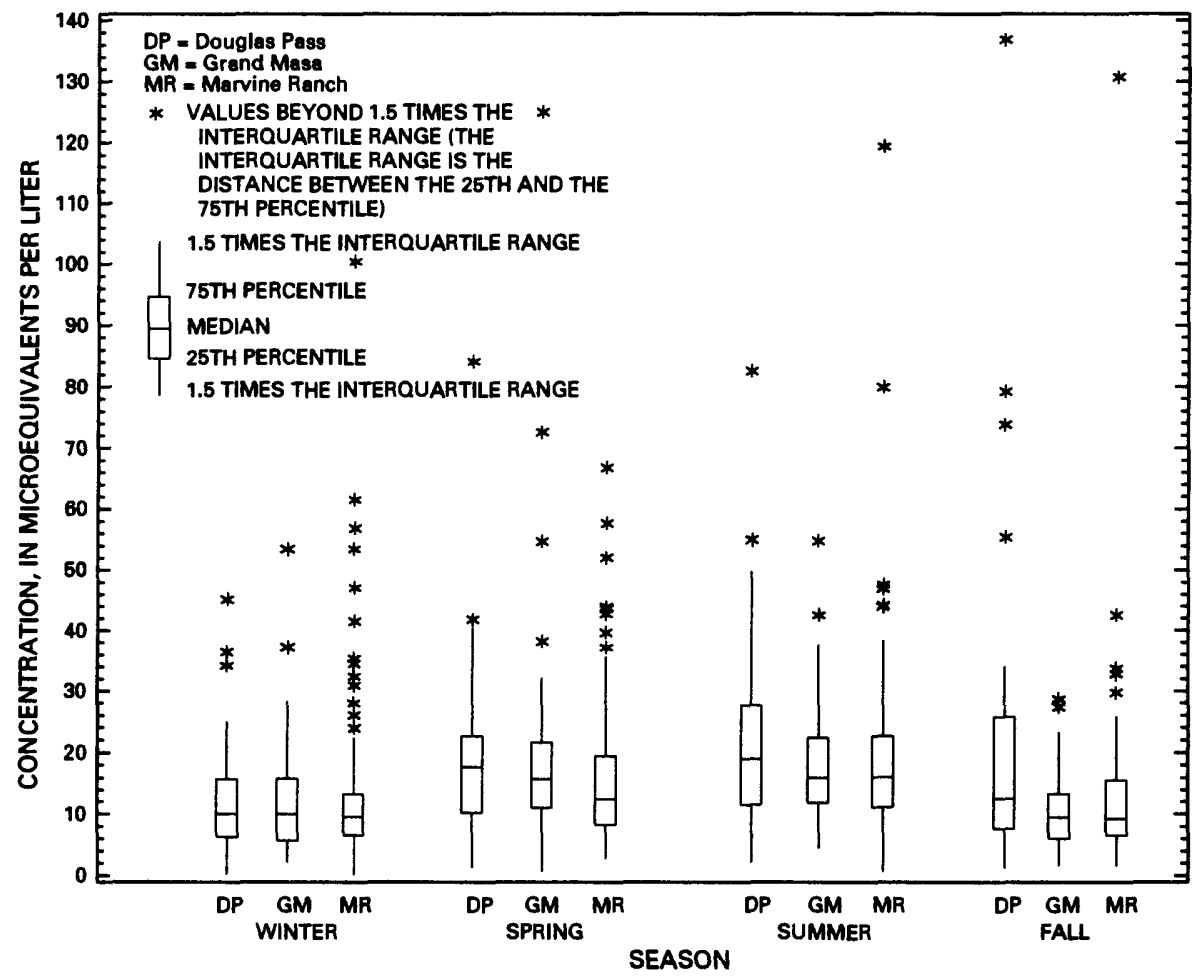

Figure 9. Seasonal variability of nitrate.

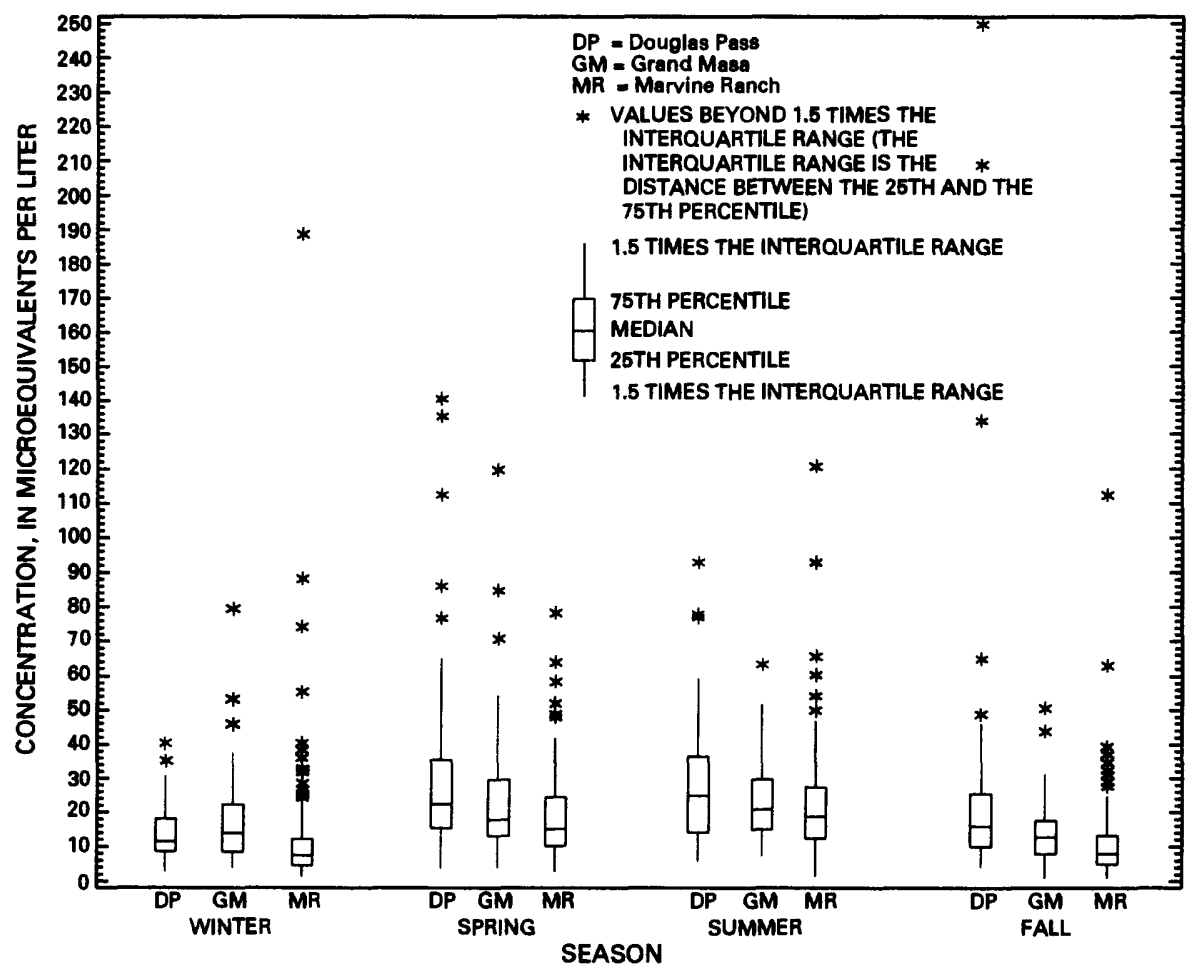

Figure 10. Seasonal variability of sulfate. 


\section{REFERENCES CITED}

Fishman, M.J., and Friedman, L.C., (eds.), 1989, Methods for determination of inorganic substances in water and fluvial sediments: U.S. Geological Survey Techniques of Water-Resources Investigations, book 5 , chap. Al, p. 595.

Lucey, K.J., and Peart, D.B., 1988, Quality-assurance data for routine water analysis in the laboratories of the U.S. Geological Survey for water year 1985: U.S. Geological Survey Water-Resources Investigations Report 88-4109.

1989a, Quality-assurance data for routine water analysis in the laboratories of the U.S. Geological Survey for water year 1986: U.S. Geological Survey Water-Resources Investigations Report 89-4009. $-1989 \mathrm{~b}$, Quality-assurance data for routine water analysis in the laboratories of the U.S. Geological Survey for water year 1987: U.S. Geological Survey Water-Resources Investigations Report 89-4049. 1989 c, Quality-assurance data for routine water analysis in the laboratories of the U.S. Geological Survey for water year 1988: U.S. Geological Survey Water-Resources Investigations Report 89-4166.
Maloney, T.J., Ludtke, A.S., and Krizman, T.L., 1992, Quality-assurance data for routine water analysis in the laboratories of the U.S. Geological Survey for water year 1989: U.S. Geological Survey Water-Resources Investigations Report 92-4075.

1993, Quality-assurance data for routine water analysis in the laboratories of the U.S. Geological Survey for water year 1990: U.S. Geological Survey Water-Resources Investigations Report 93-4082.

1994, Quality-assurance data for routine water analysis in the laboratories of the U.S. Geological Survey for water year 1991: U.S. Geological Survey Water-Resources Investigations Report 94-4046.

Peart, D.B., and Sutphin, H.B., 1987, Quality-assurance data for routine water analysis in the laboratories of the U.S. Geological Survey for water year 1984: U.S. Geological Survey Water-Resources Investigations Report 87-4077.

Turk, J.T., and Adams, D.B., 1983, Sensitivity to acidification of lakes in the Flat Tops Wilderness Area, Colorado: Water Resources Research, v. 19, no. 2, p. 346-350. 
PRECIPITATION DATA 


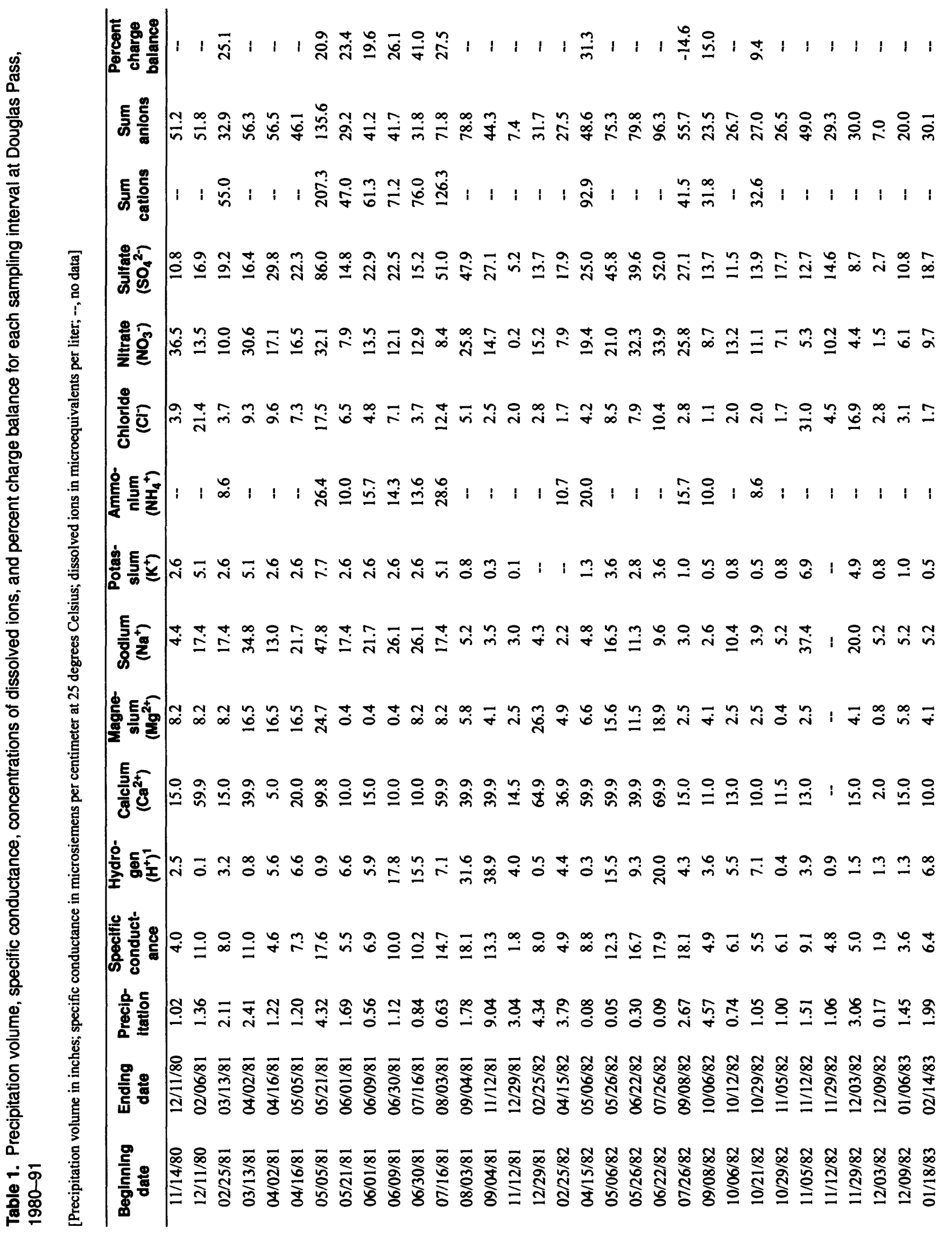




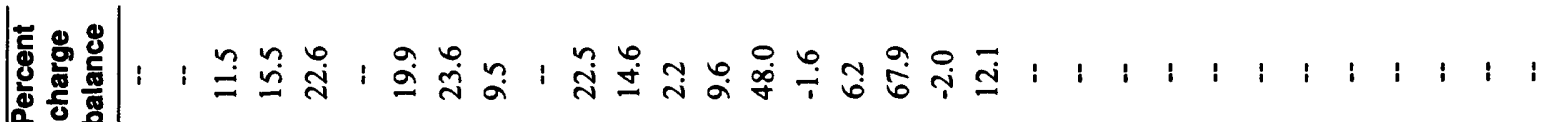

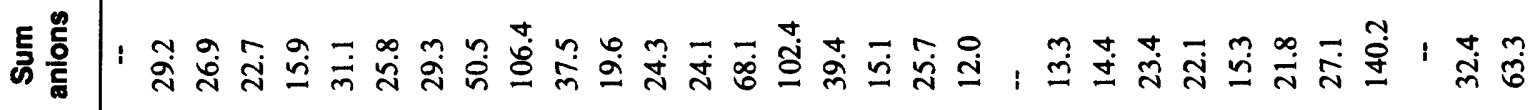
ह

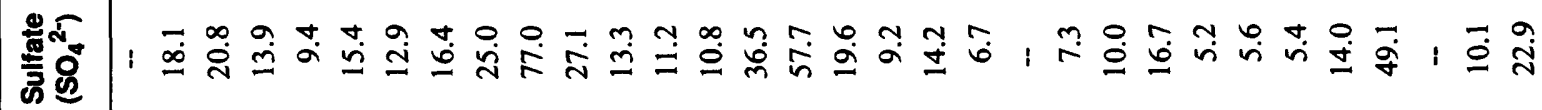
竧家

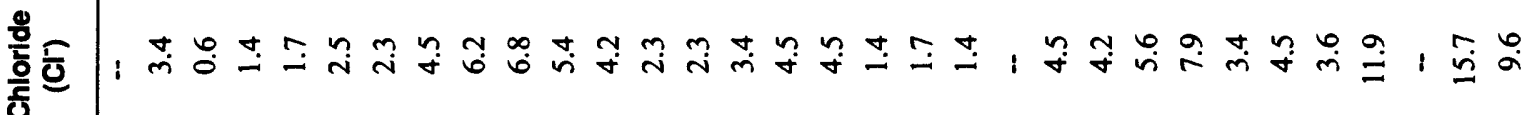

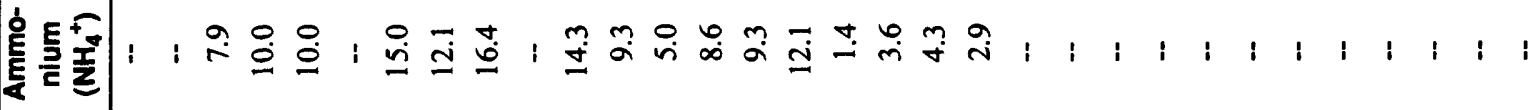

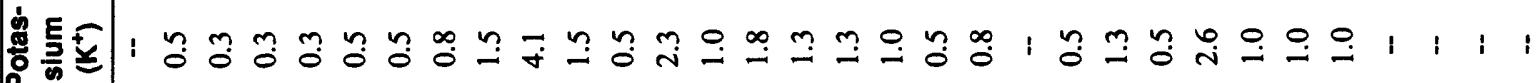

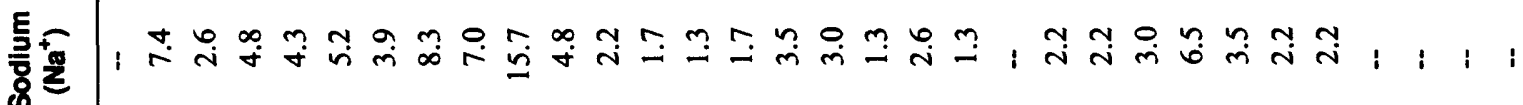

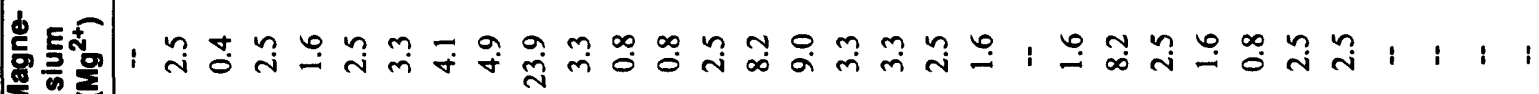
声鸷

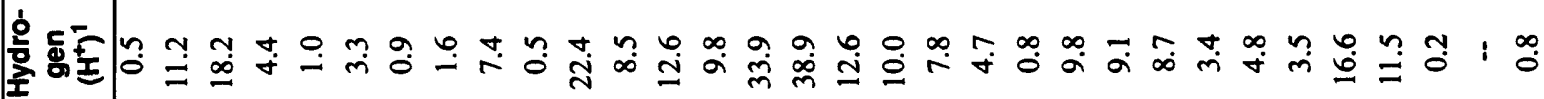

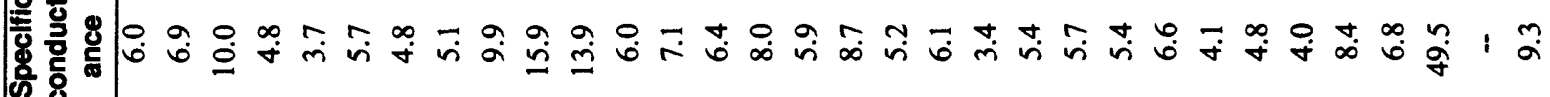
흘

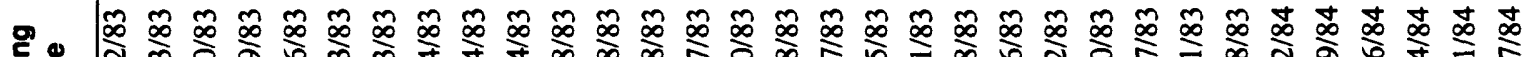
站范

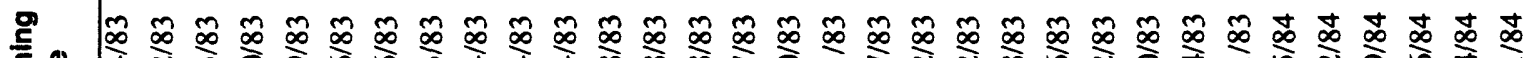

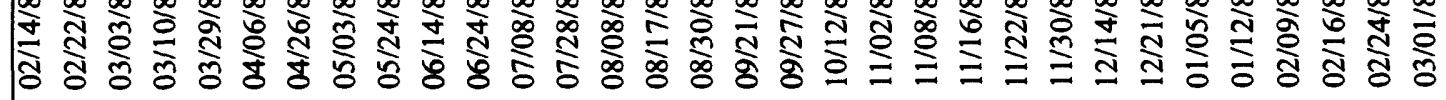




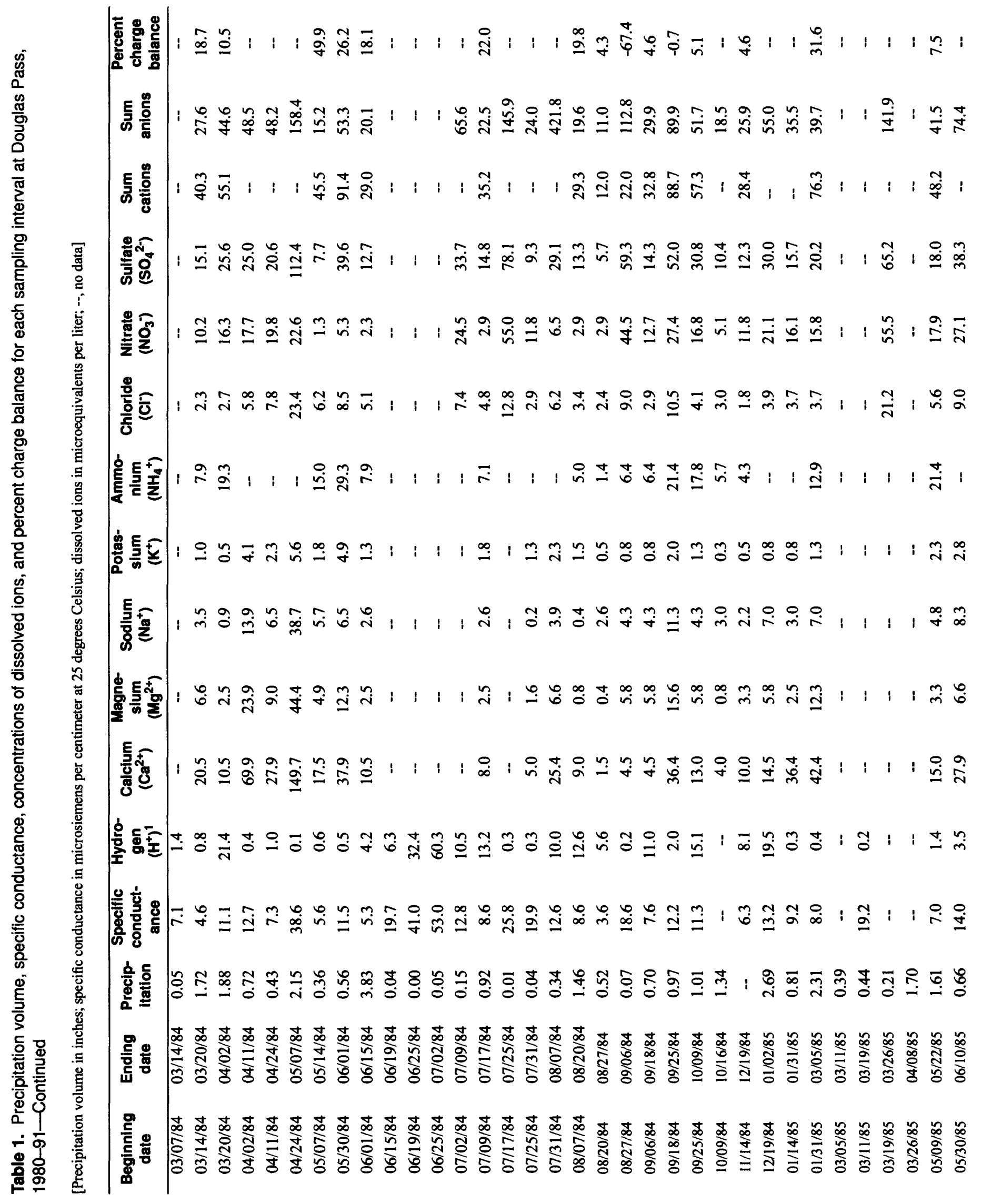




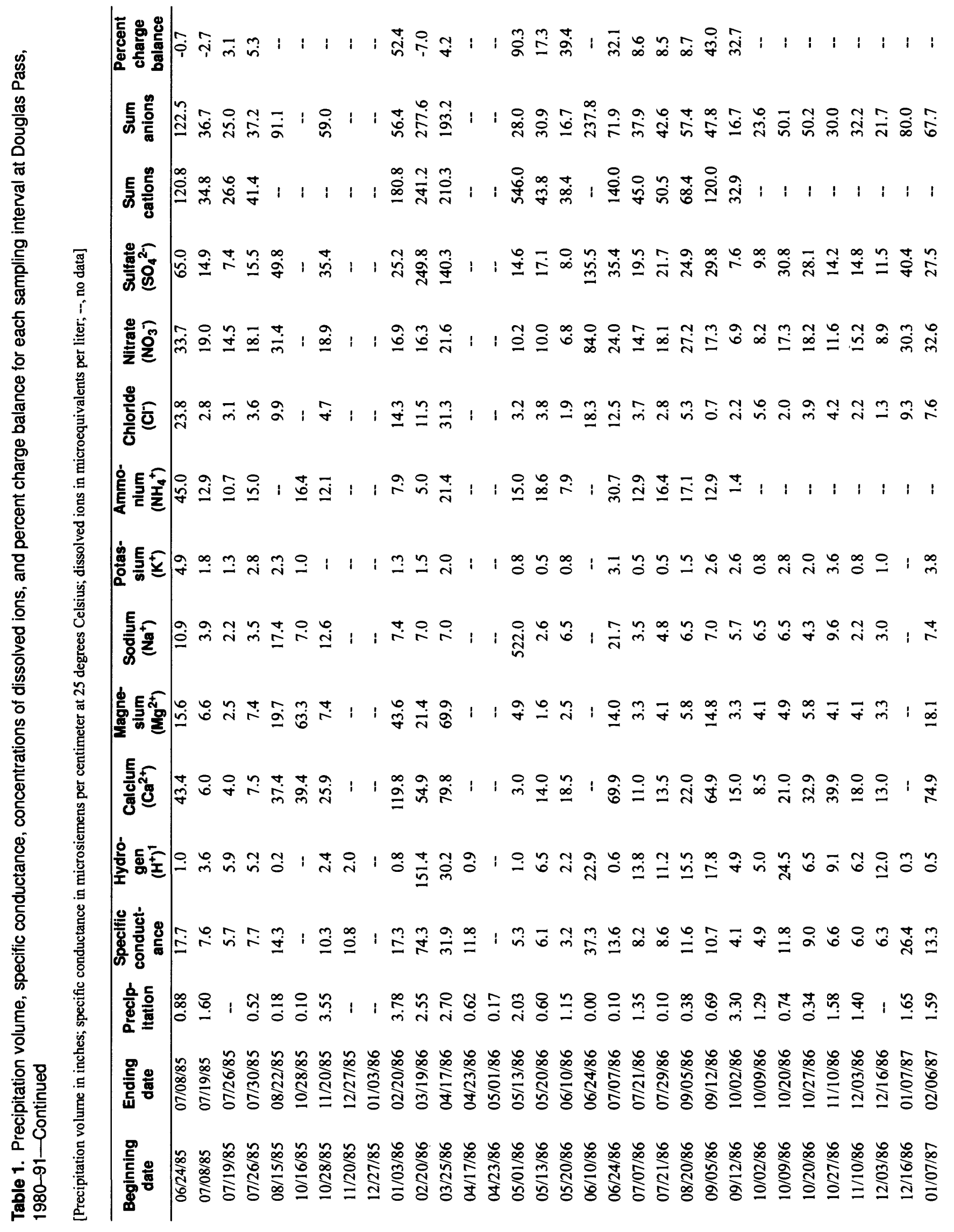




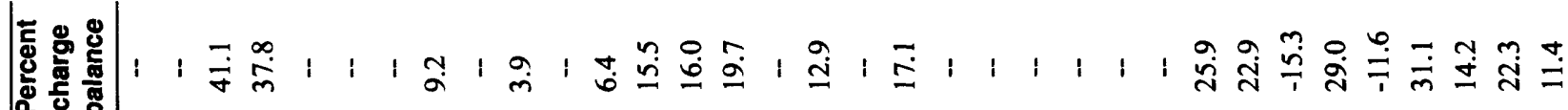

孞参

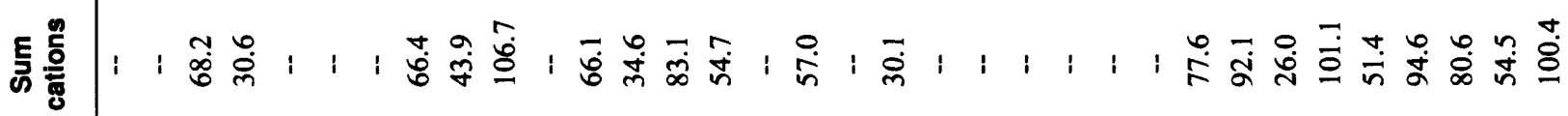

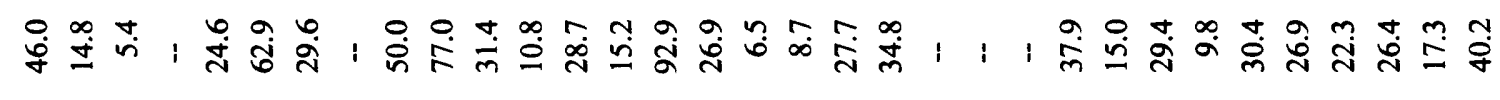
产

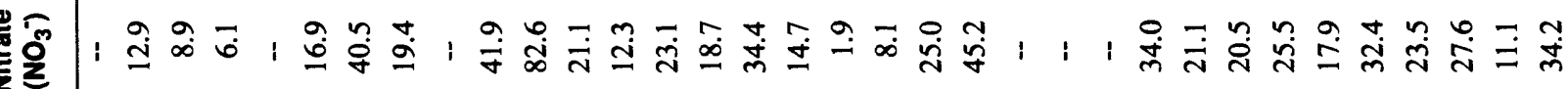

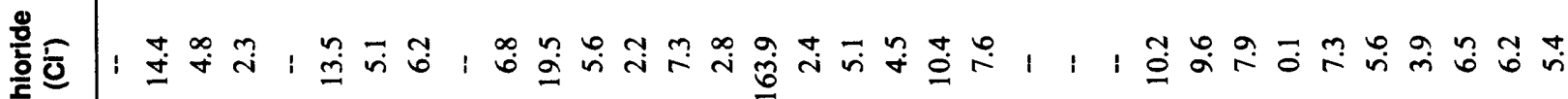
동

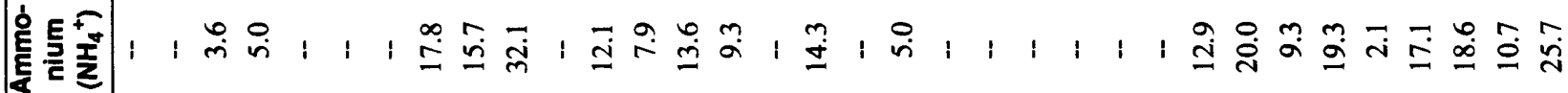

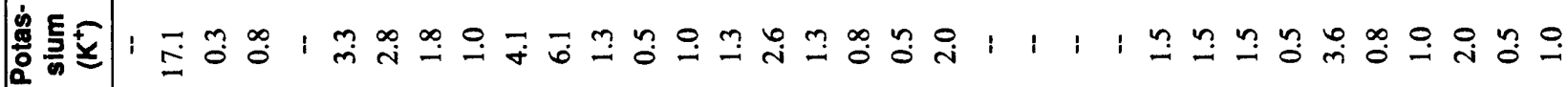
言称

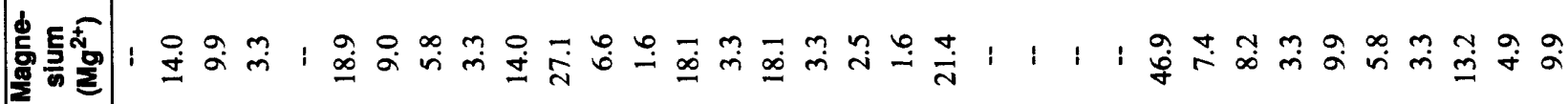

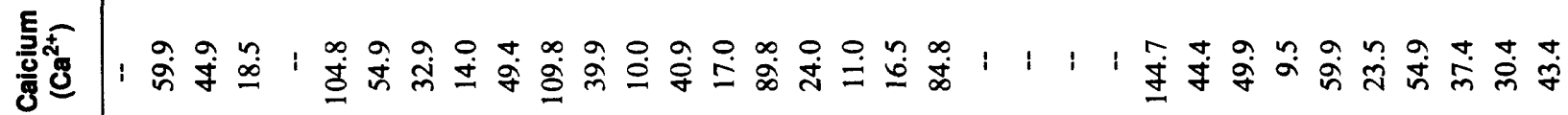

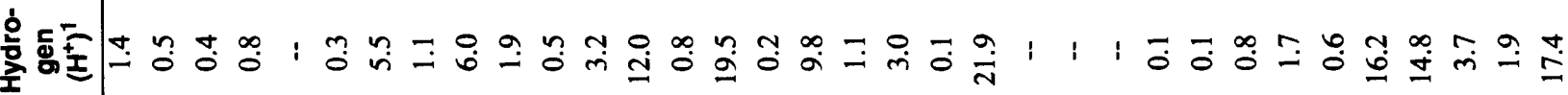
$0 \frac{1}{\mathrm{O}}$

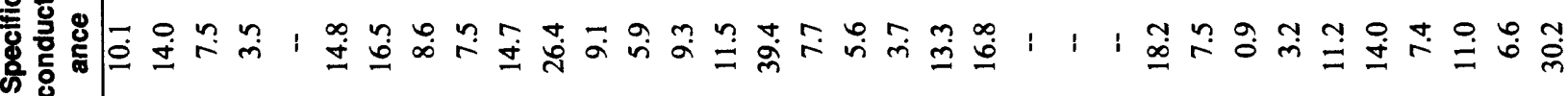

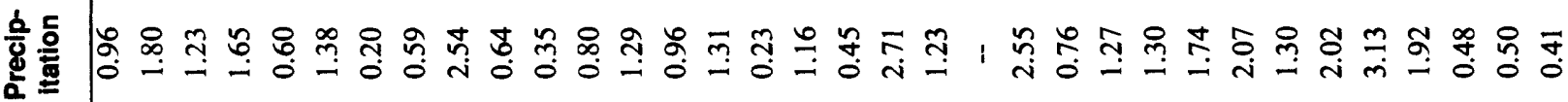

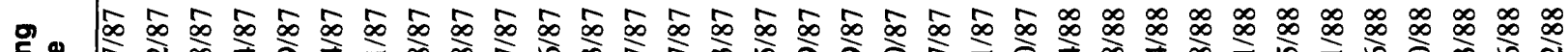

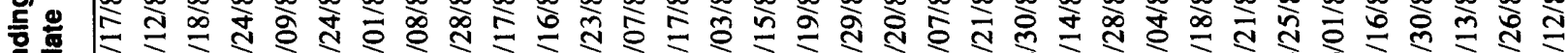

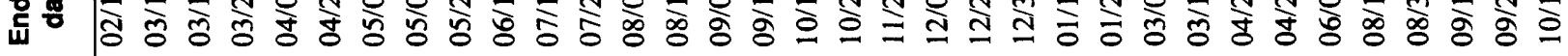

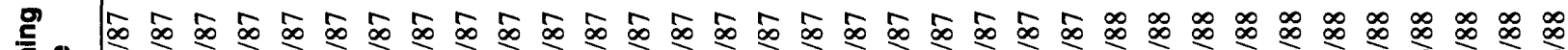

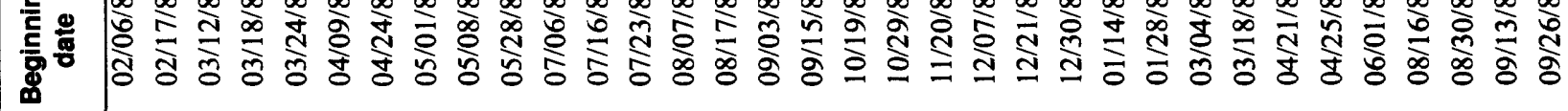




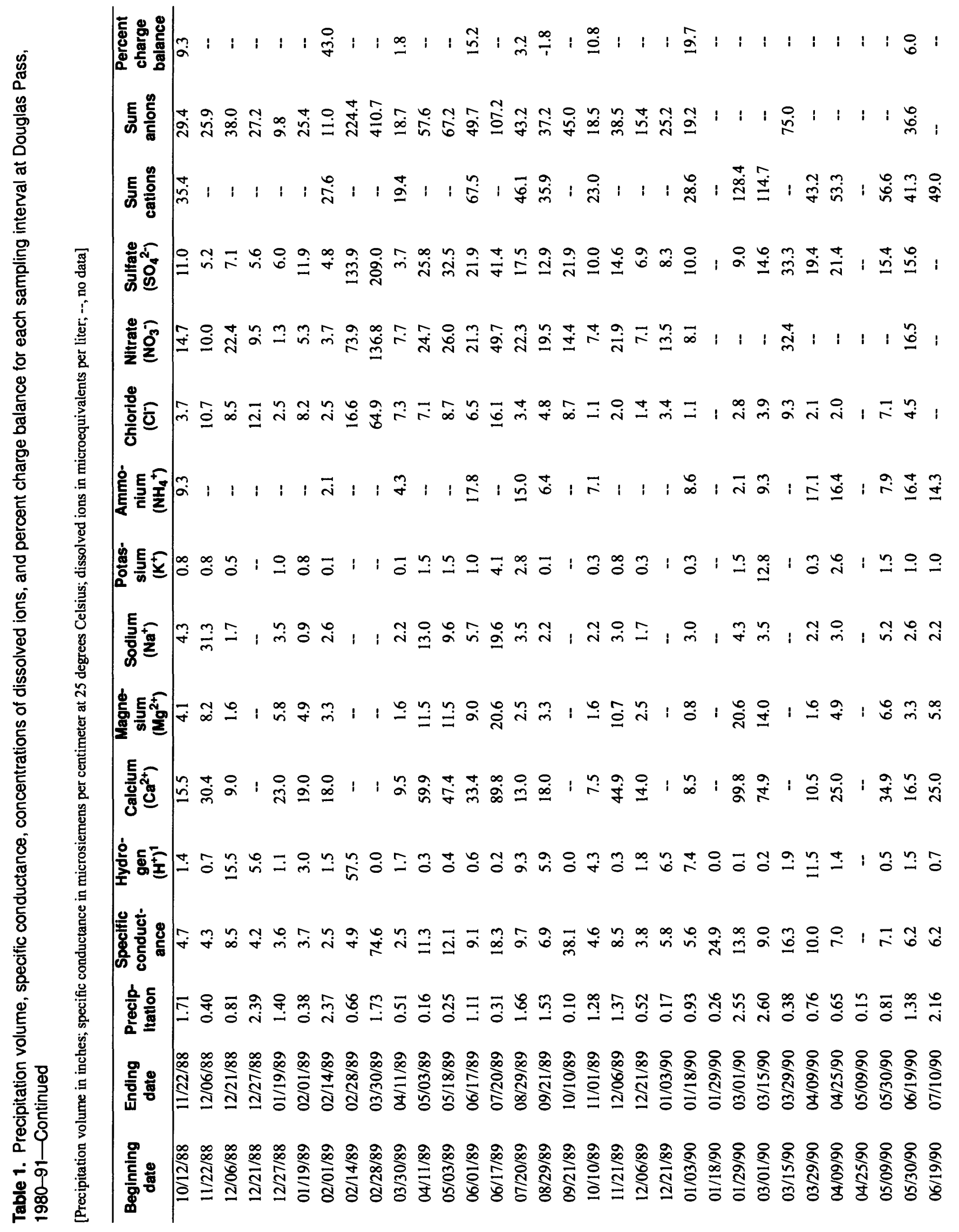




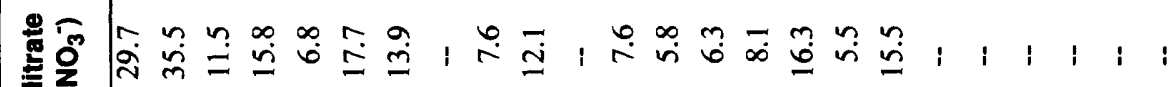

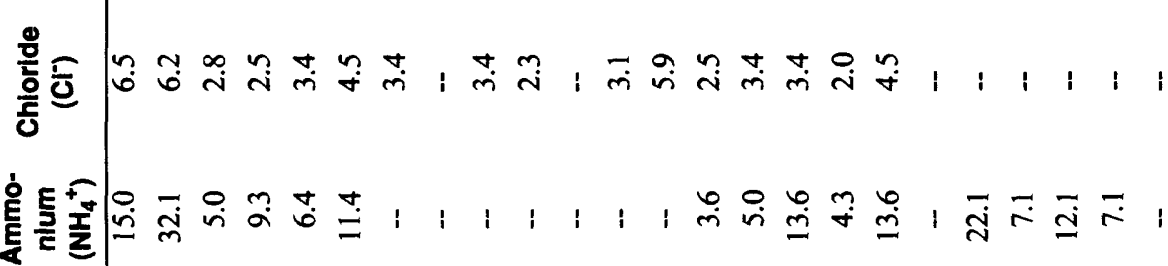

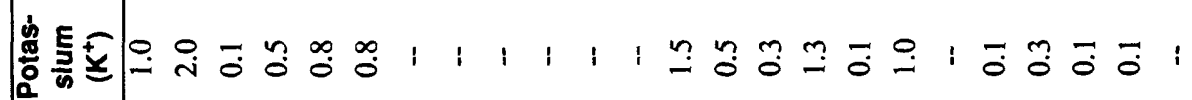

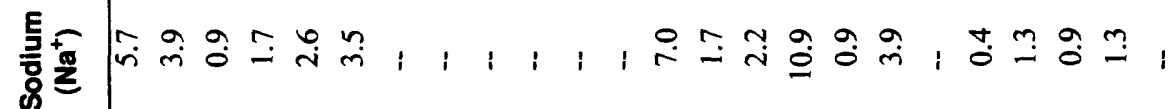

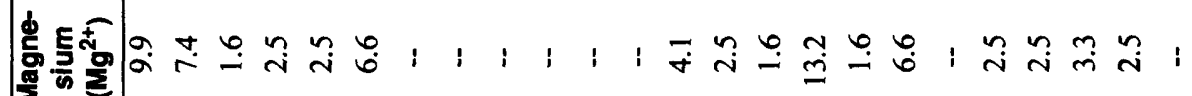
| 家

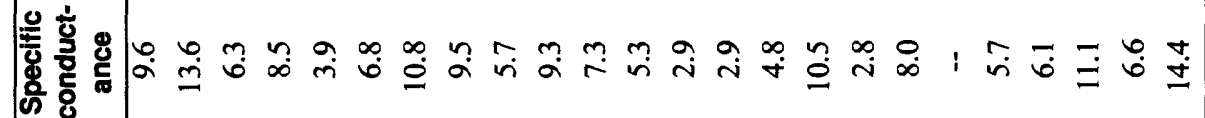

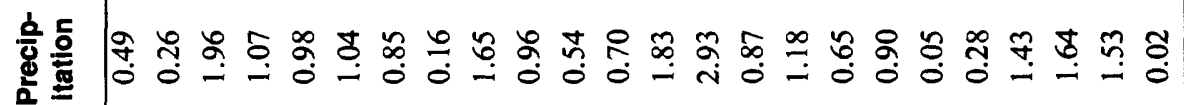

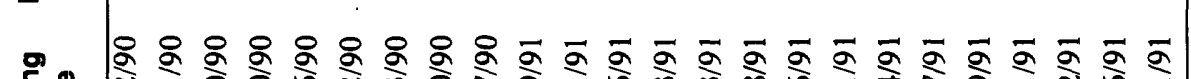

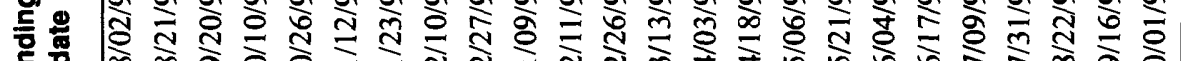

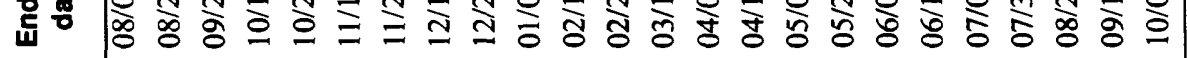

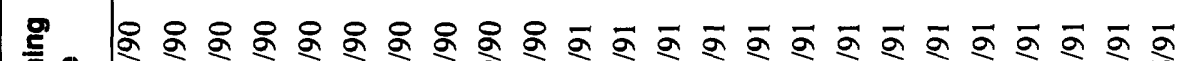

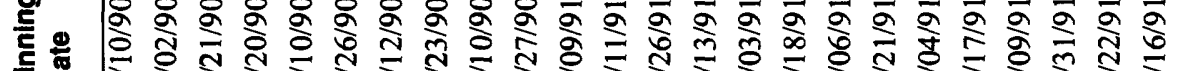
要 


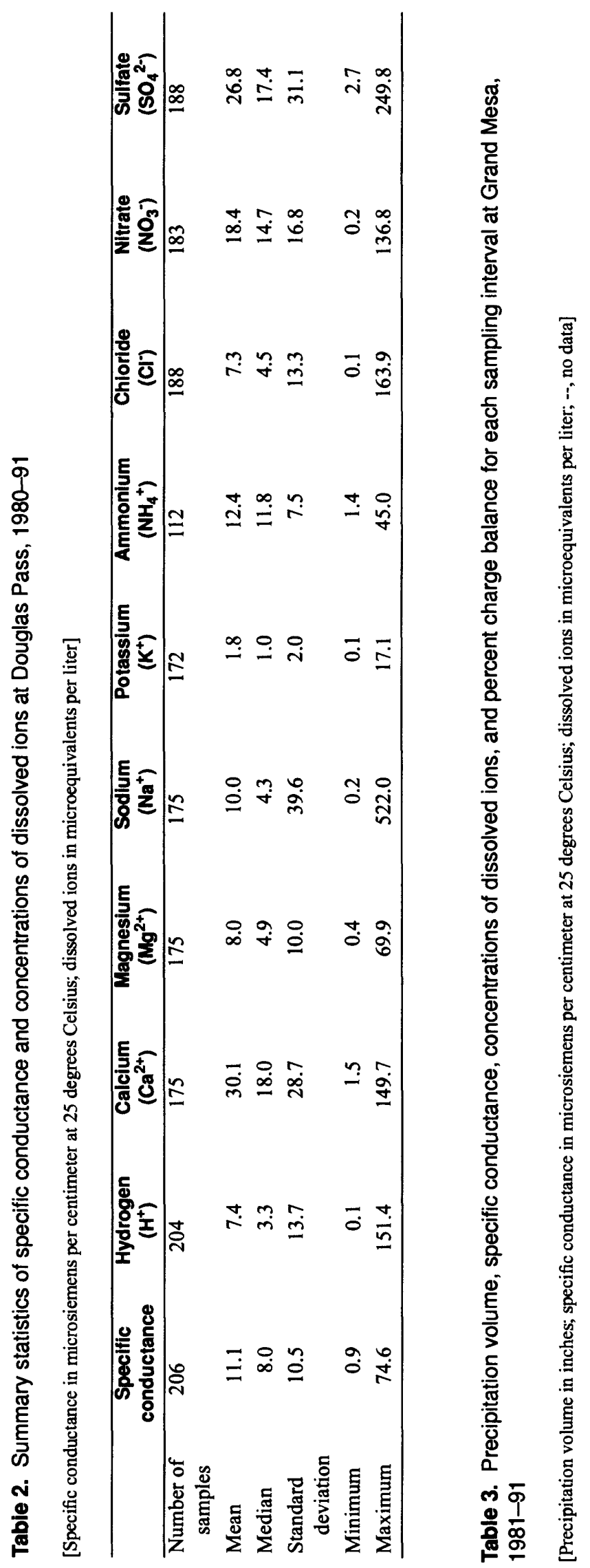

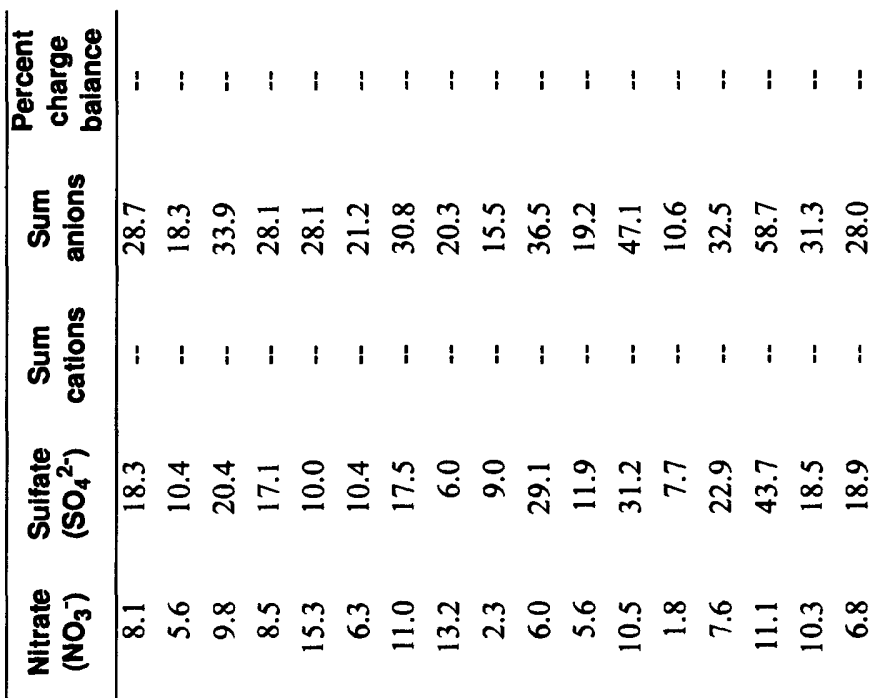

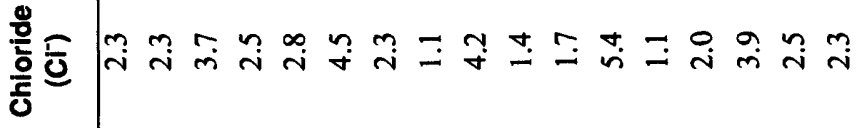

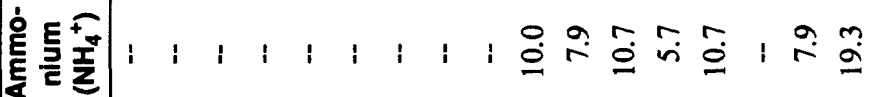

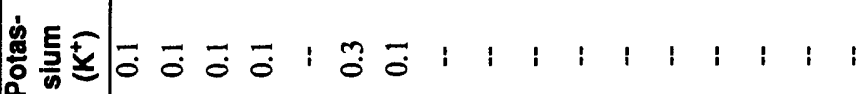
害每

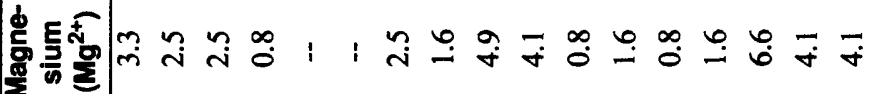
毞琶

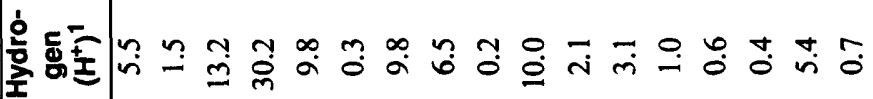
产焉 有

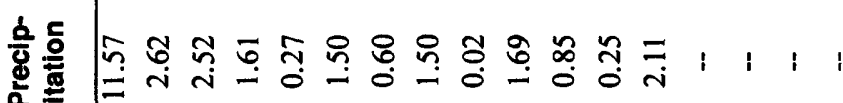

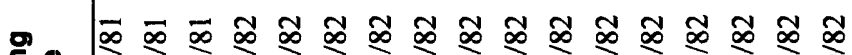

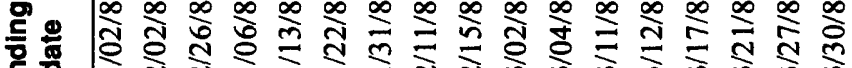

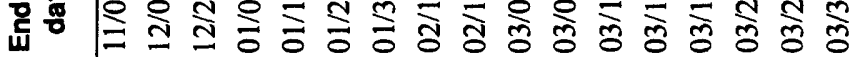
믄 産 


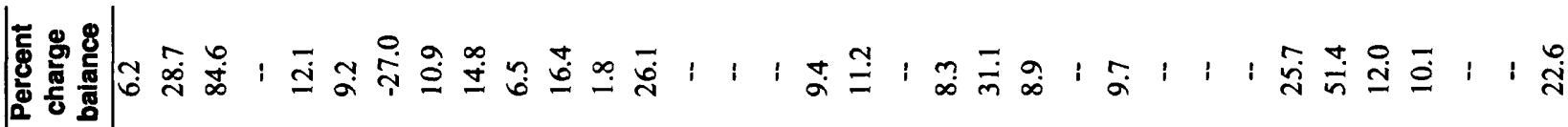

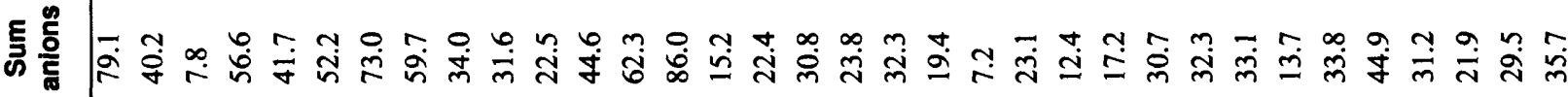
ह气

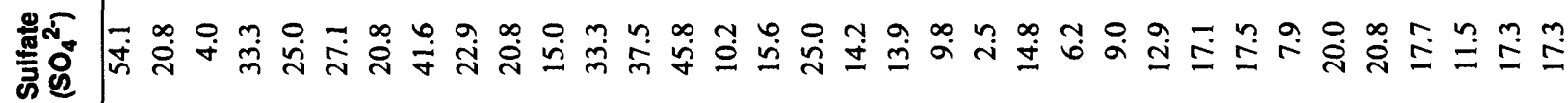
产家

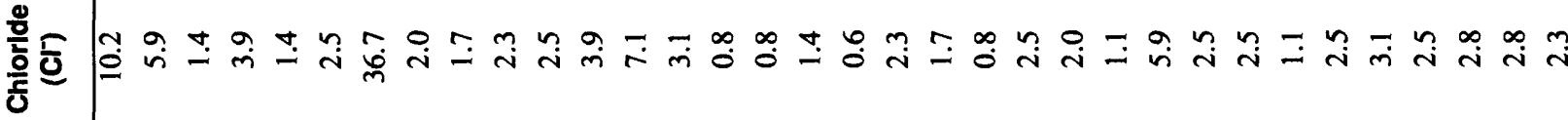

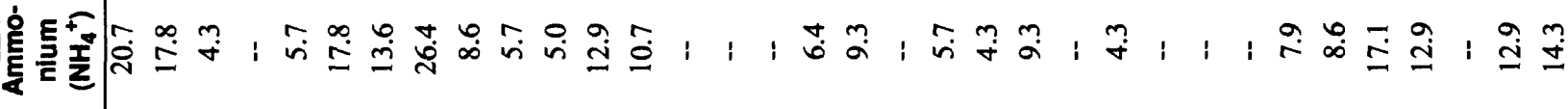

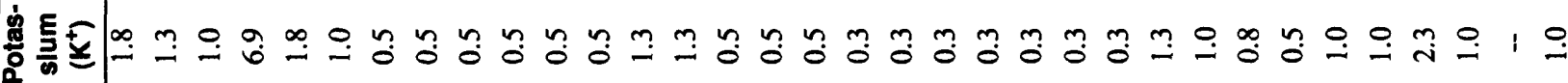
EF ठ্ु

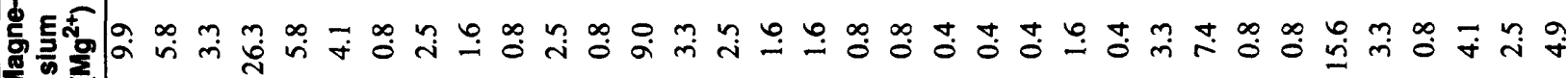

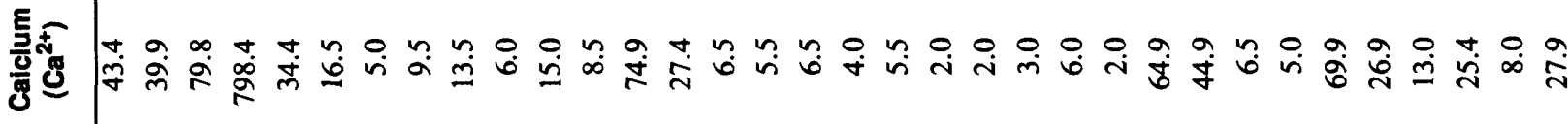

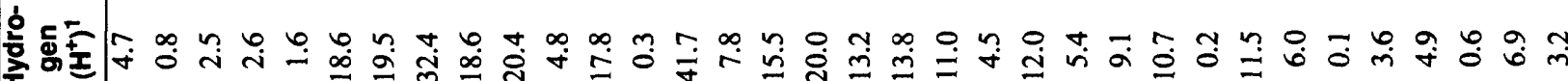

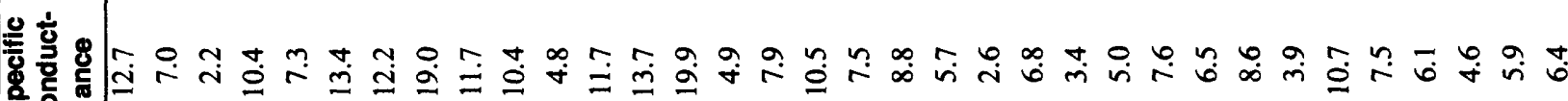
흥음 분 눈 -

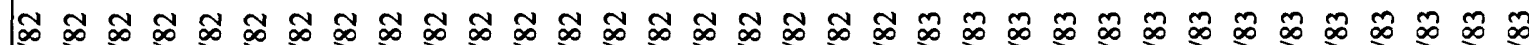
휸

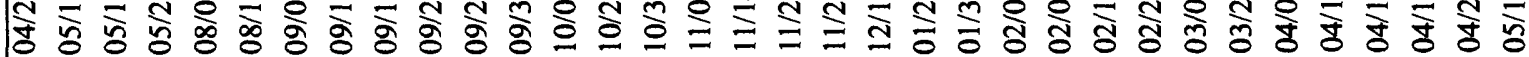

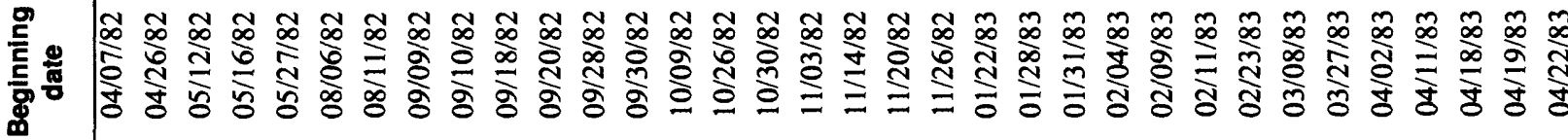




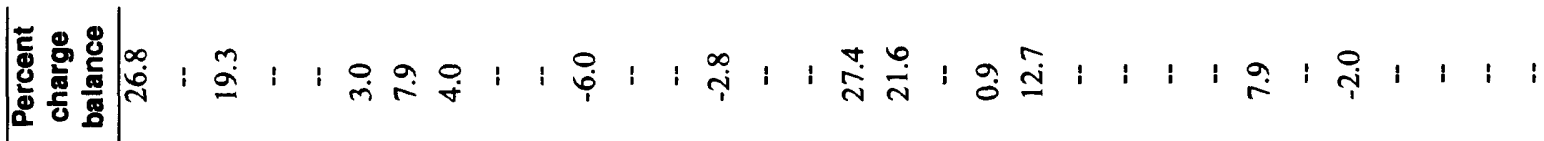

ह气 点

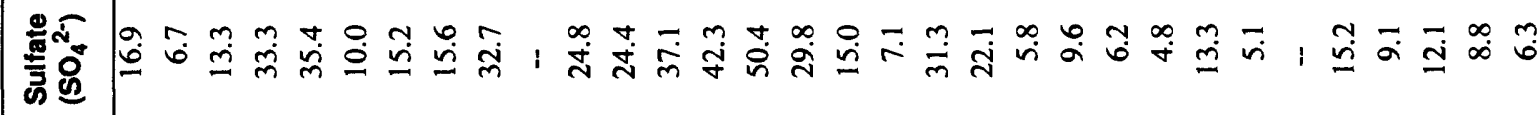
参完 흘

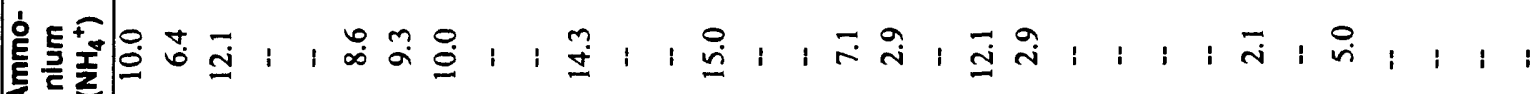

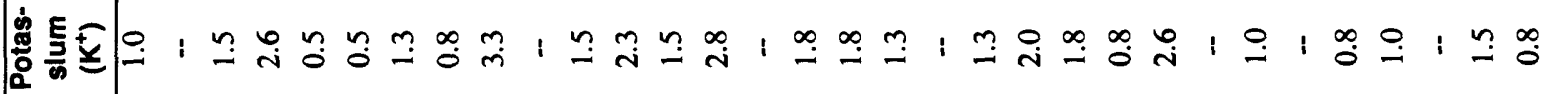
危 要

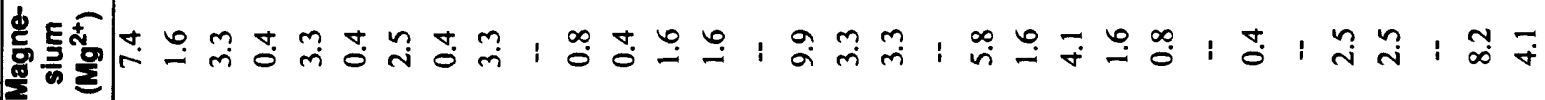

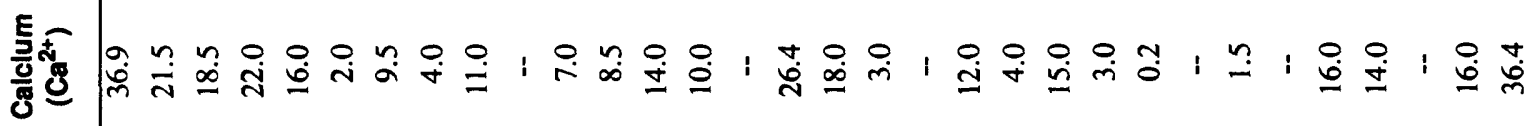
产 응

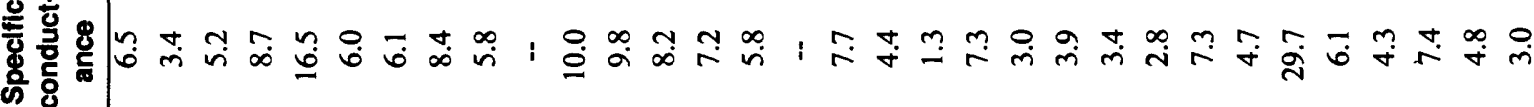

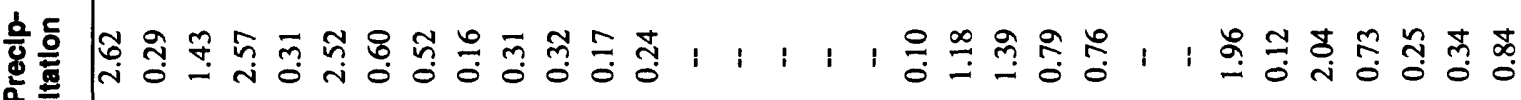

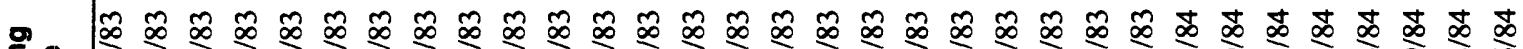

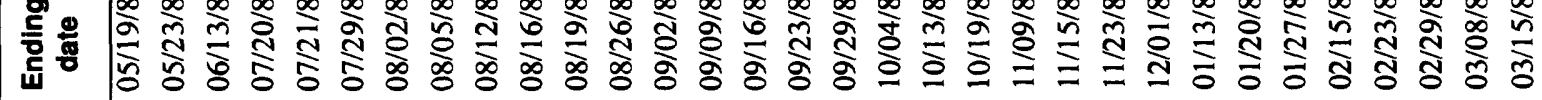

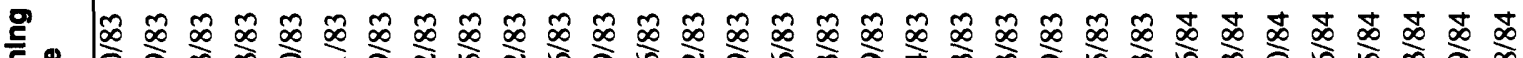
듬 布 


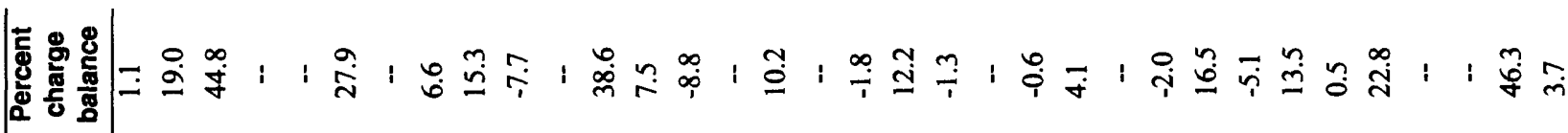

ह气 ह 焉宛 穴 ఉ. 营家 흔등

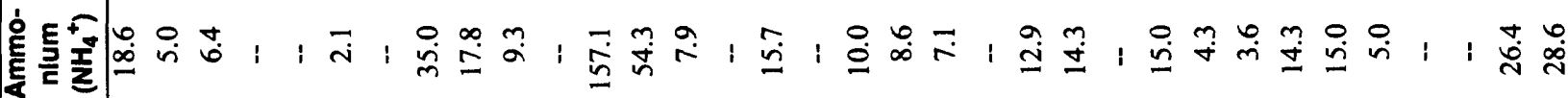

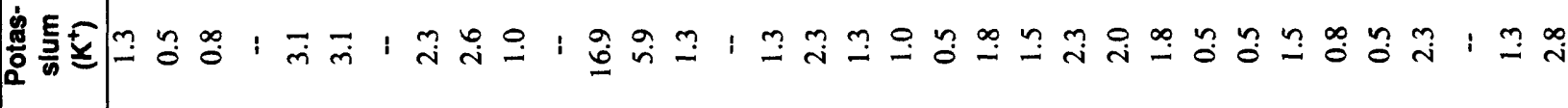

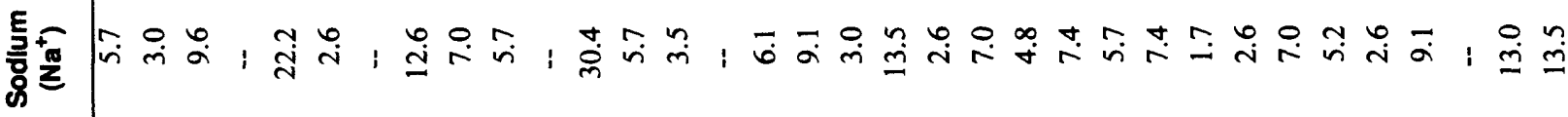

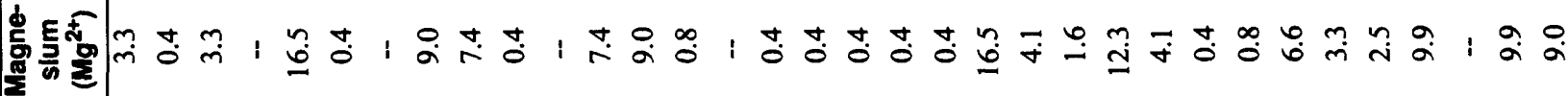

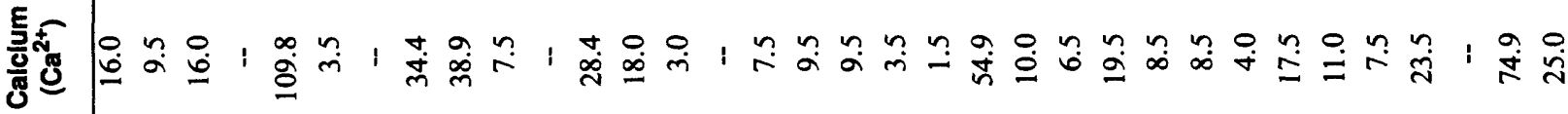

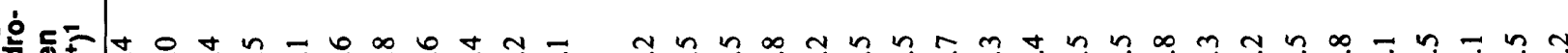

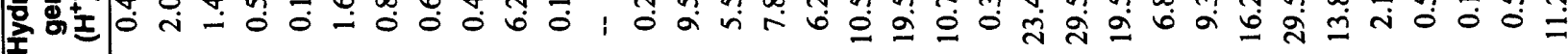

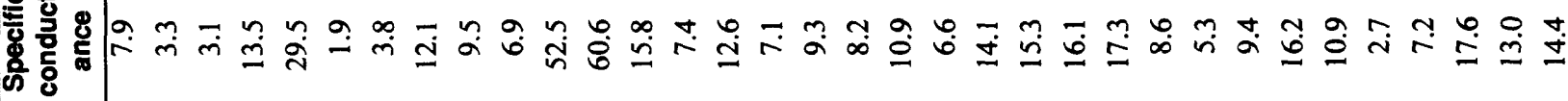

흥휴 |

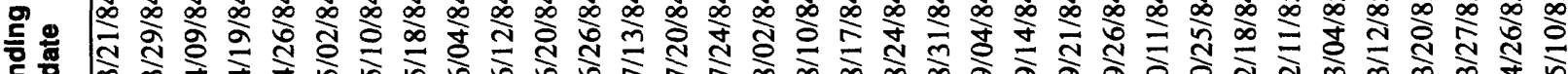

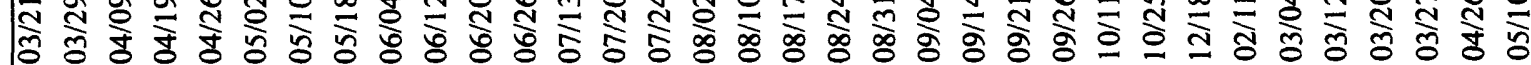

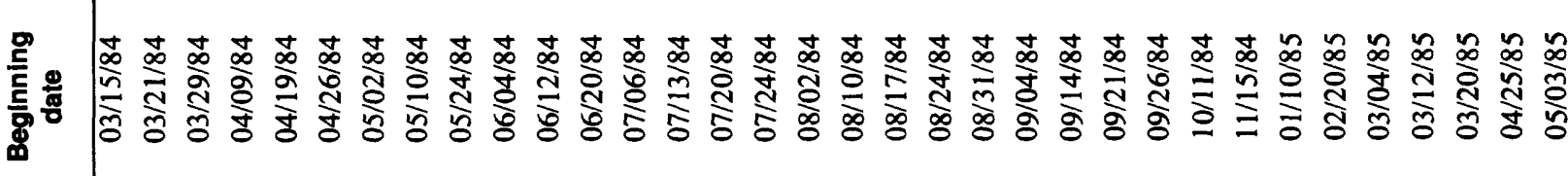




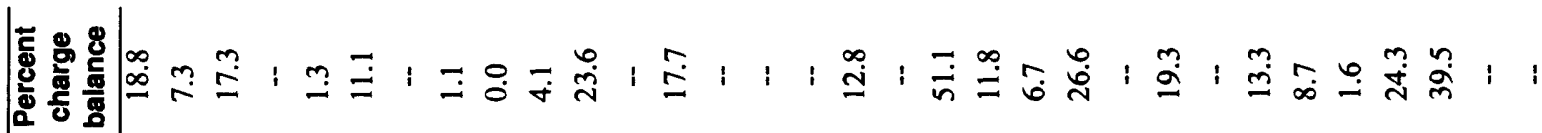

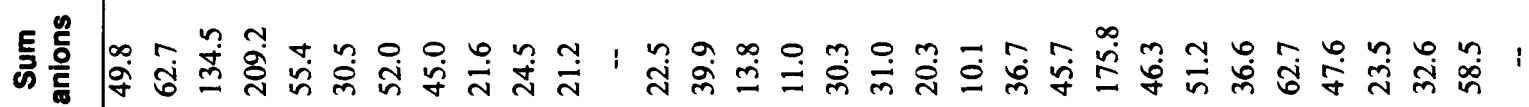
E 密灾

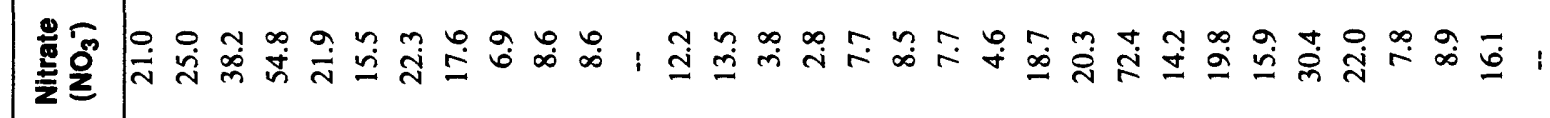

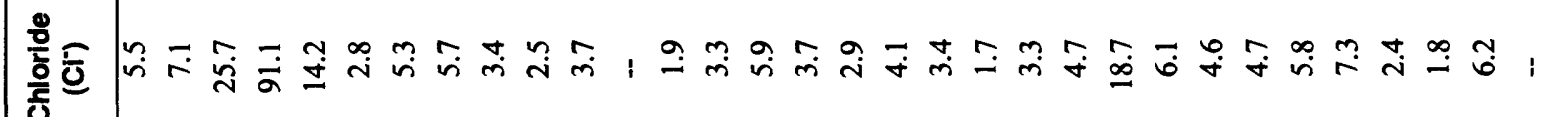

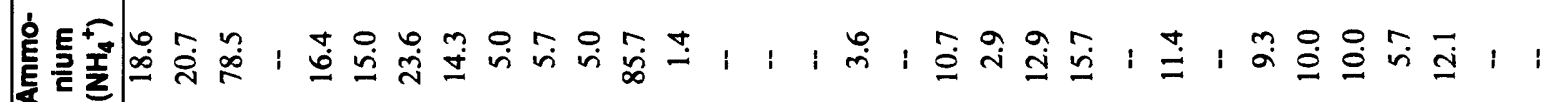

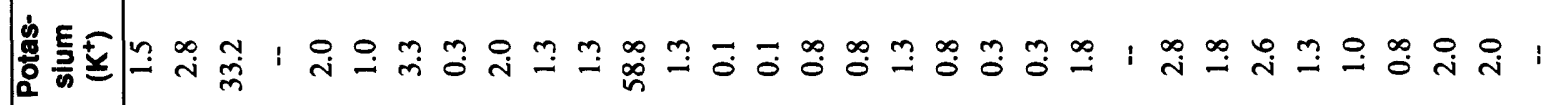

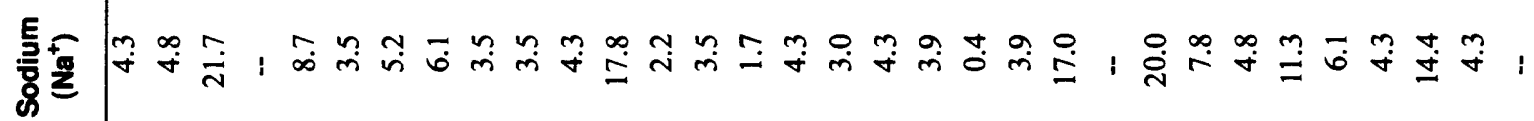

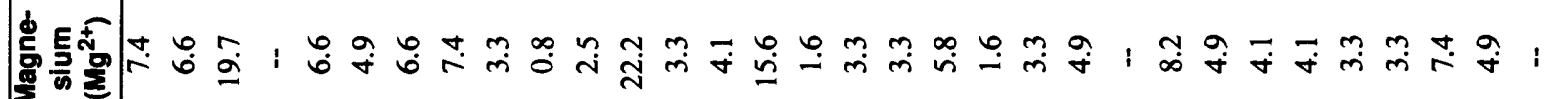

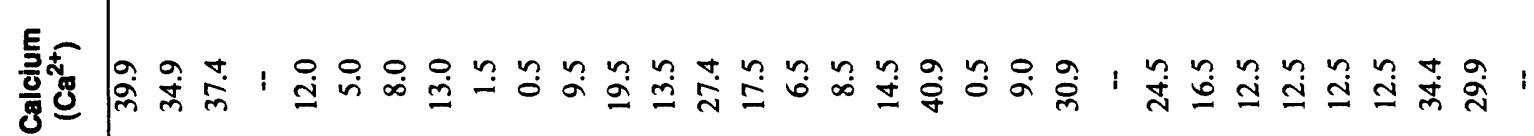

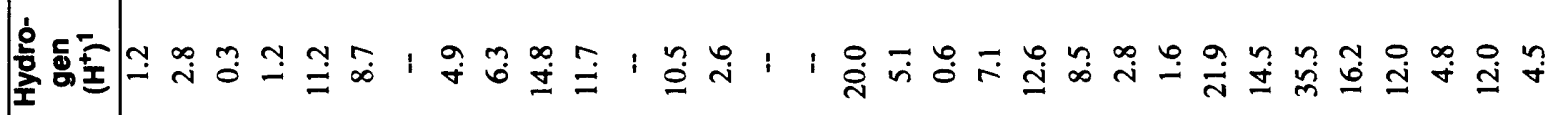

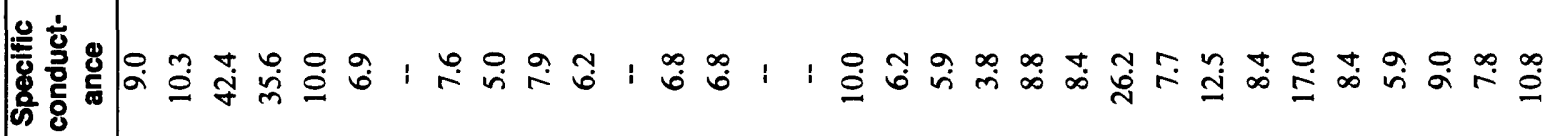
흔흫

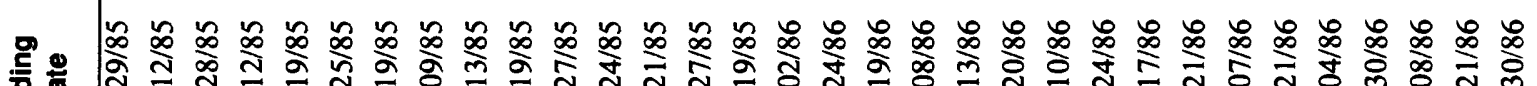

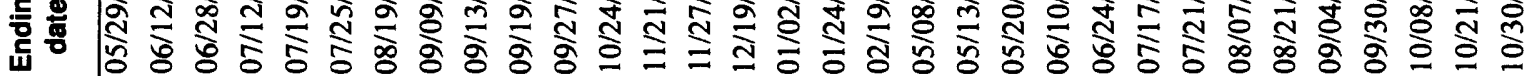

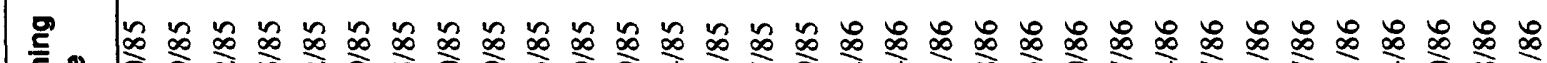
歪 


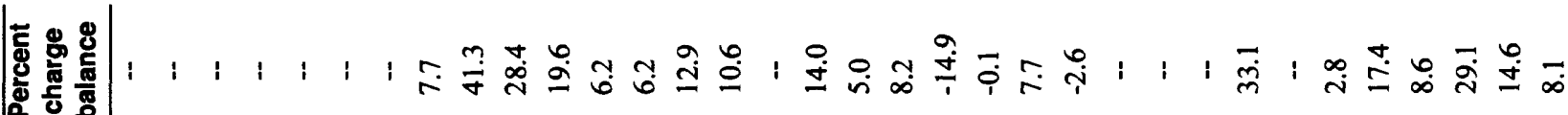

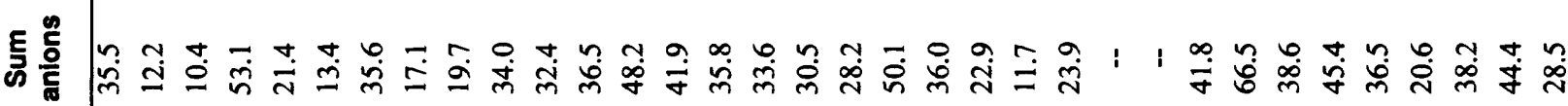

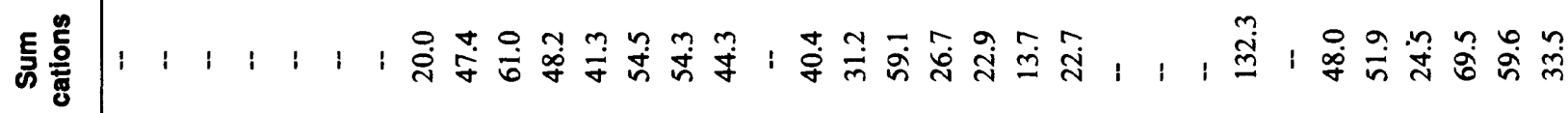
焉家 㺼家 营它

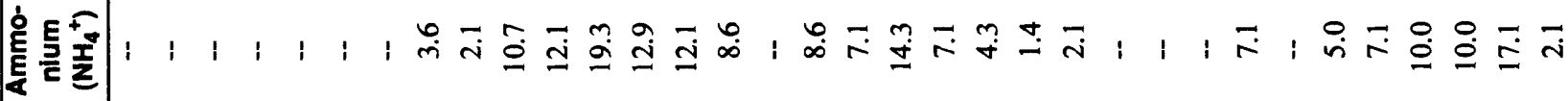

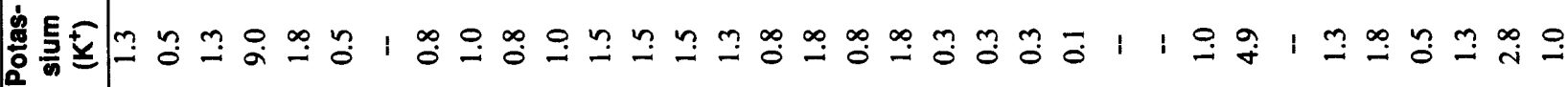

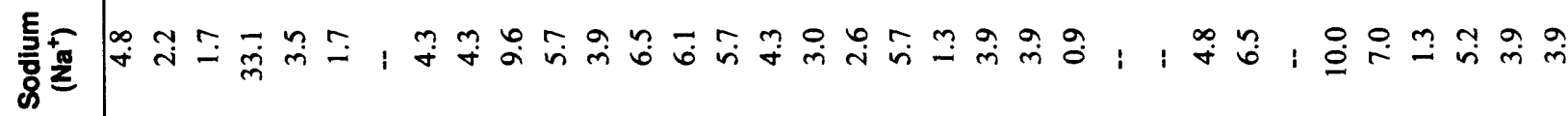

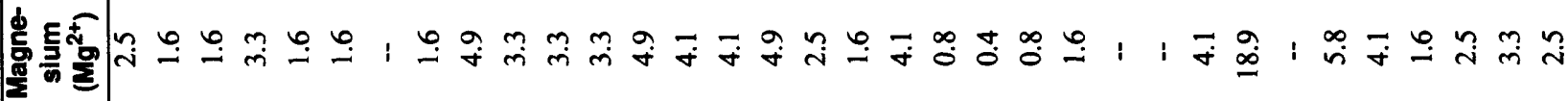

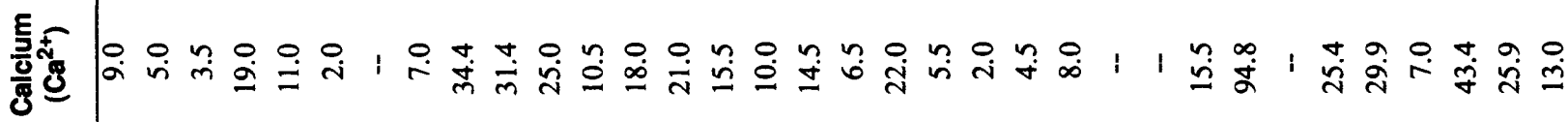

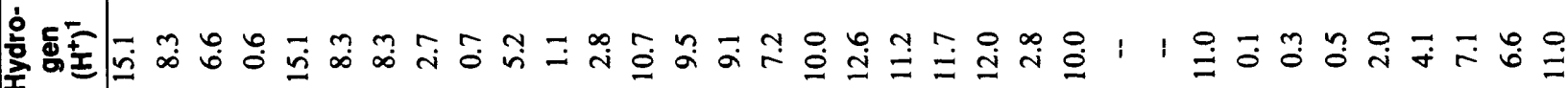

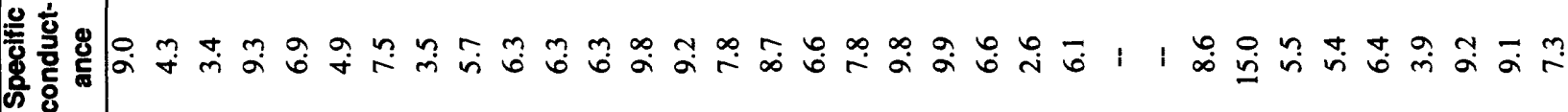

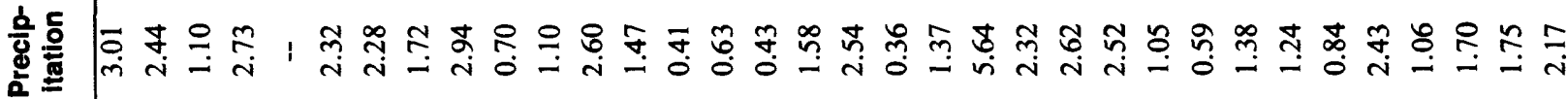
밈

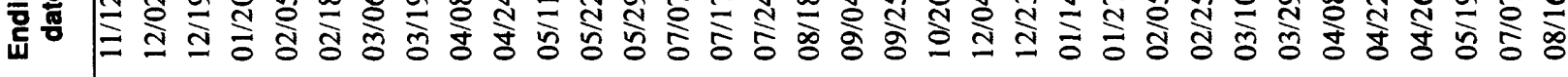

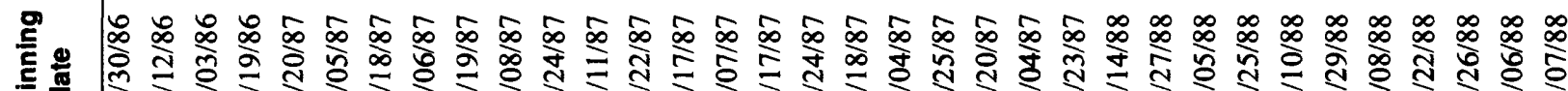

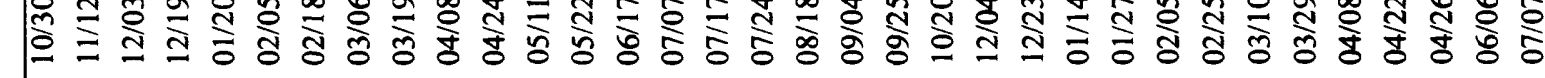




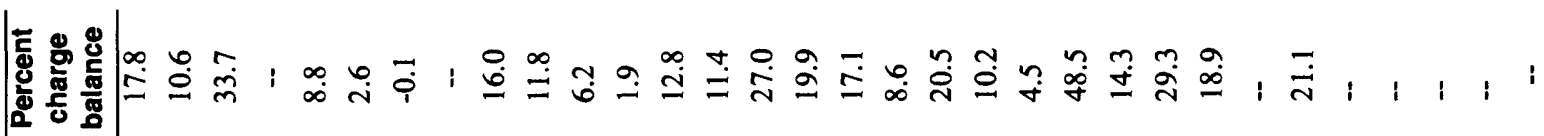

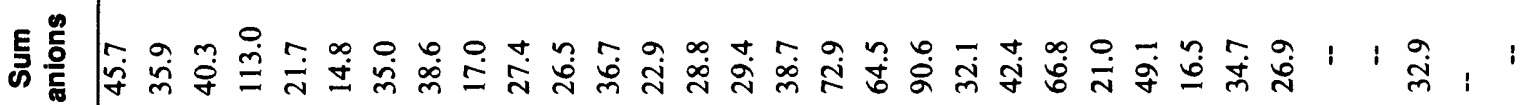

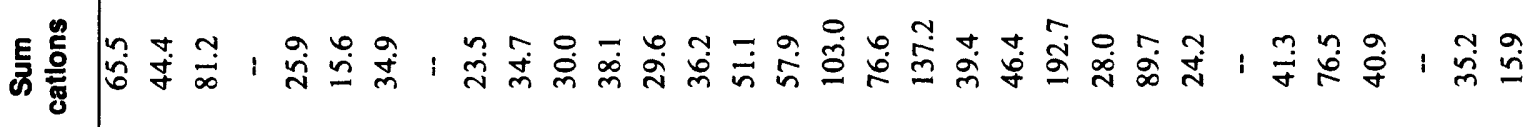

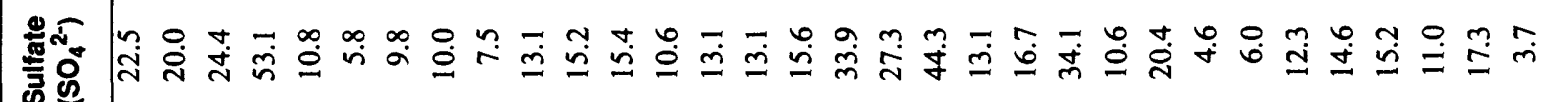
产 흥

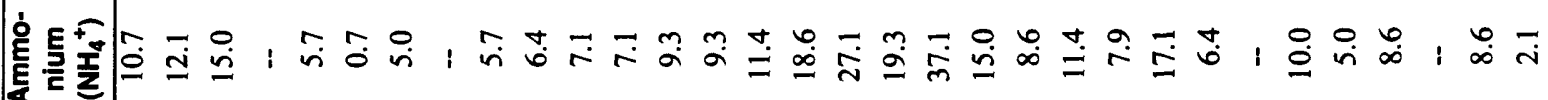
商

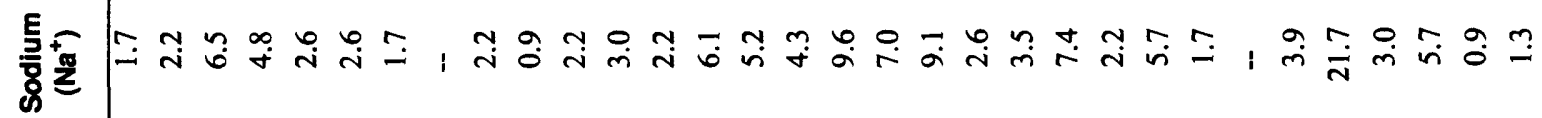

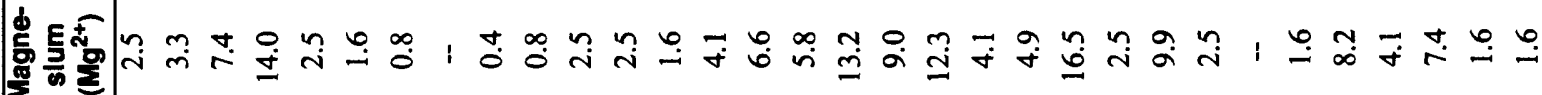
至热

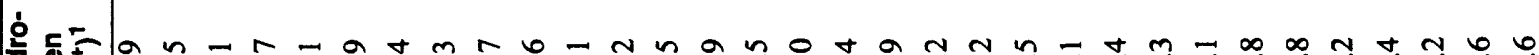

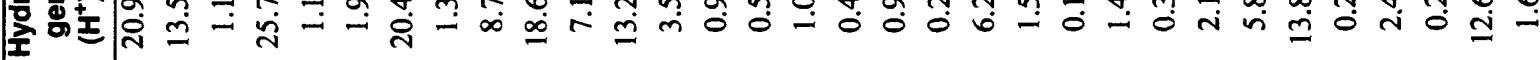

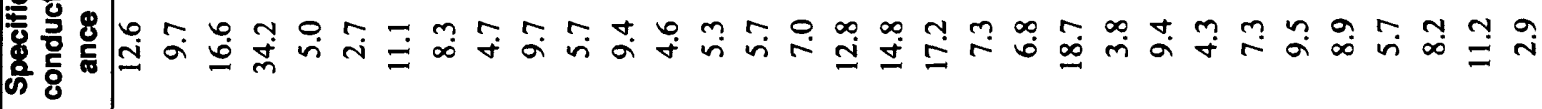

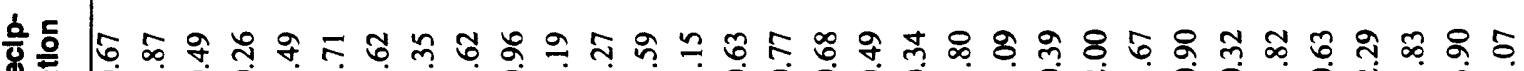

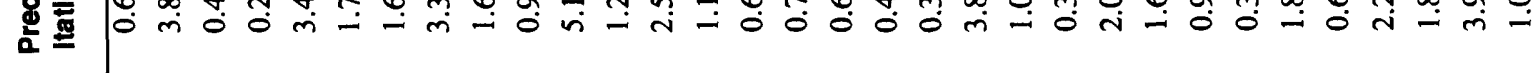

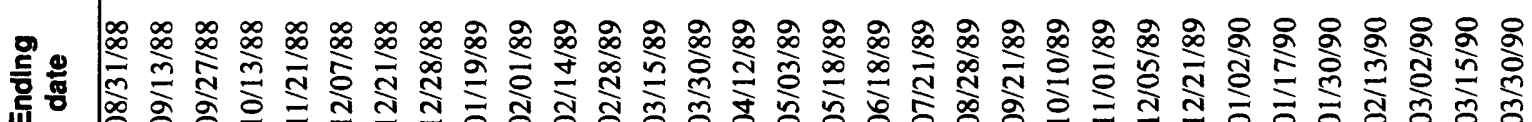

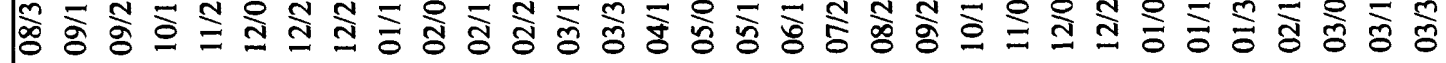

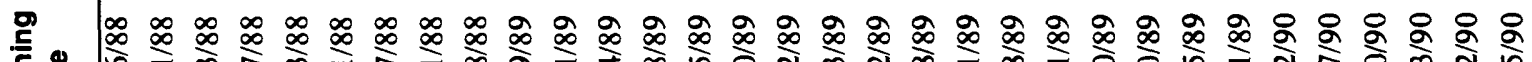
蹗要 
|

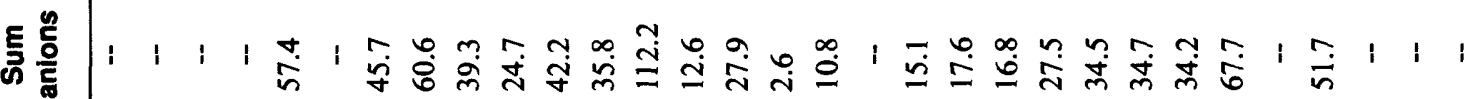
ह

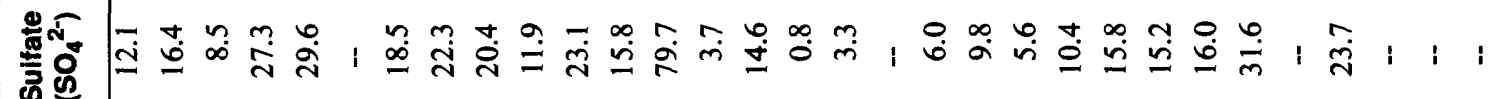

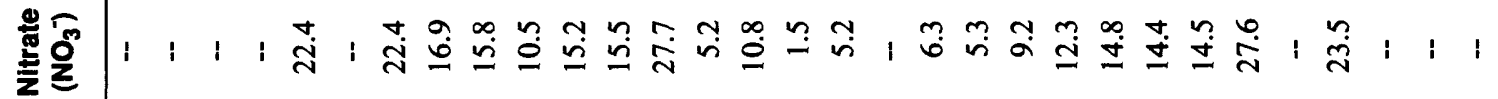

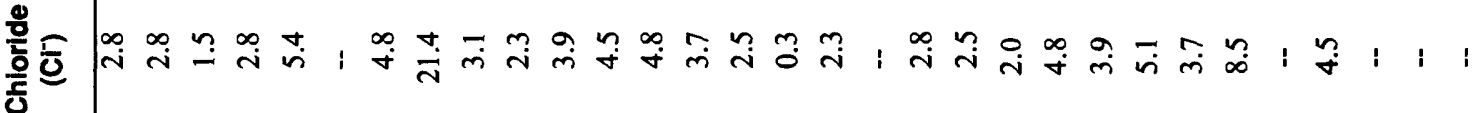

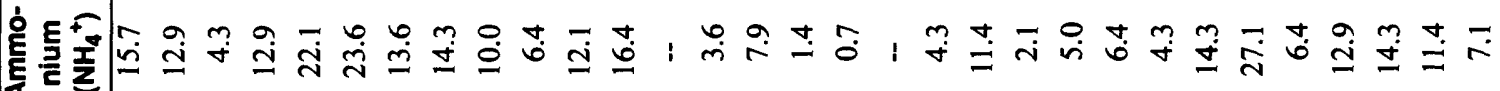

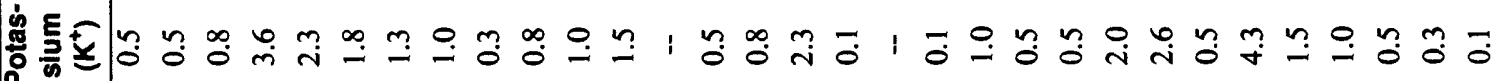

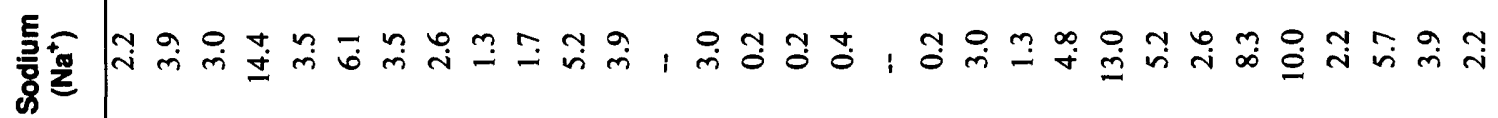

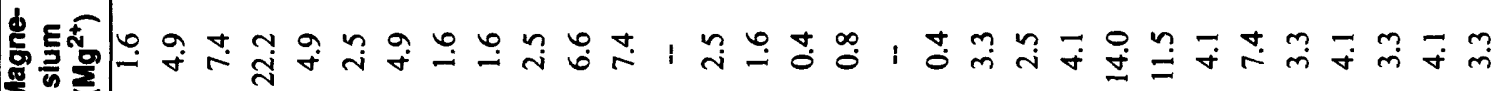

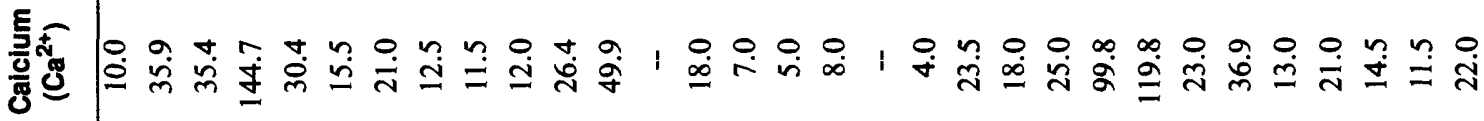

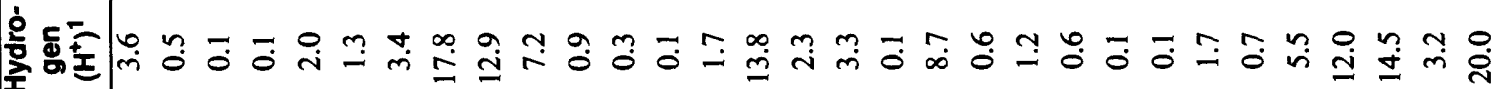

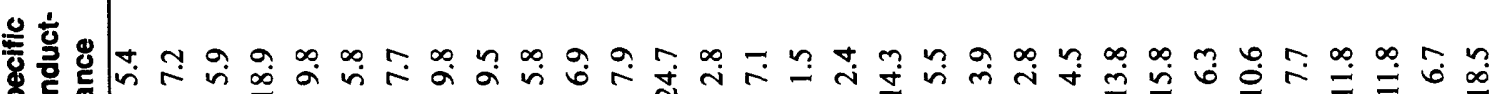
की

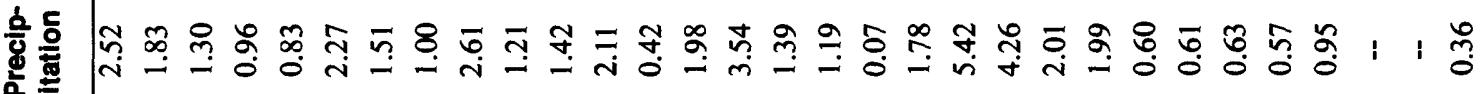

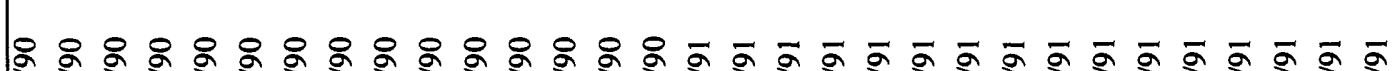

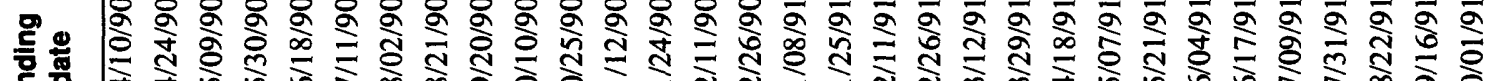

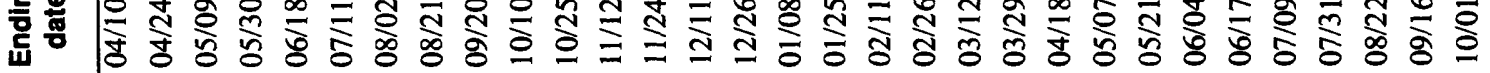

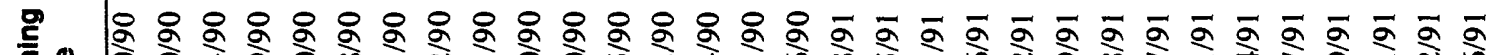

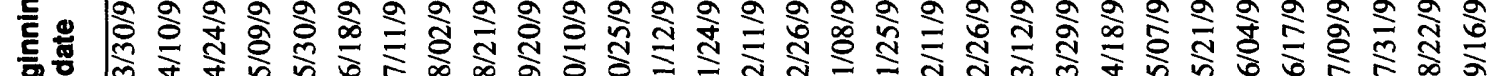

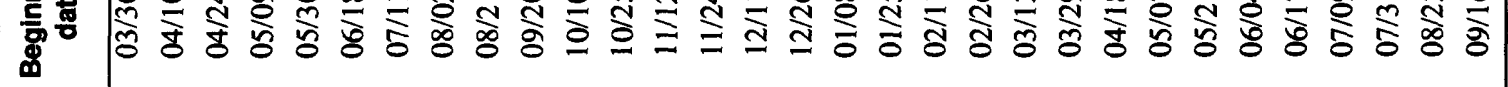




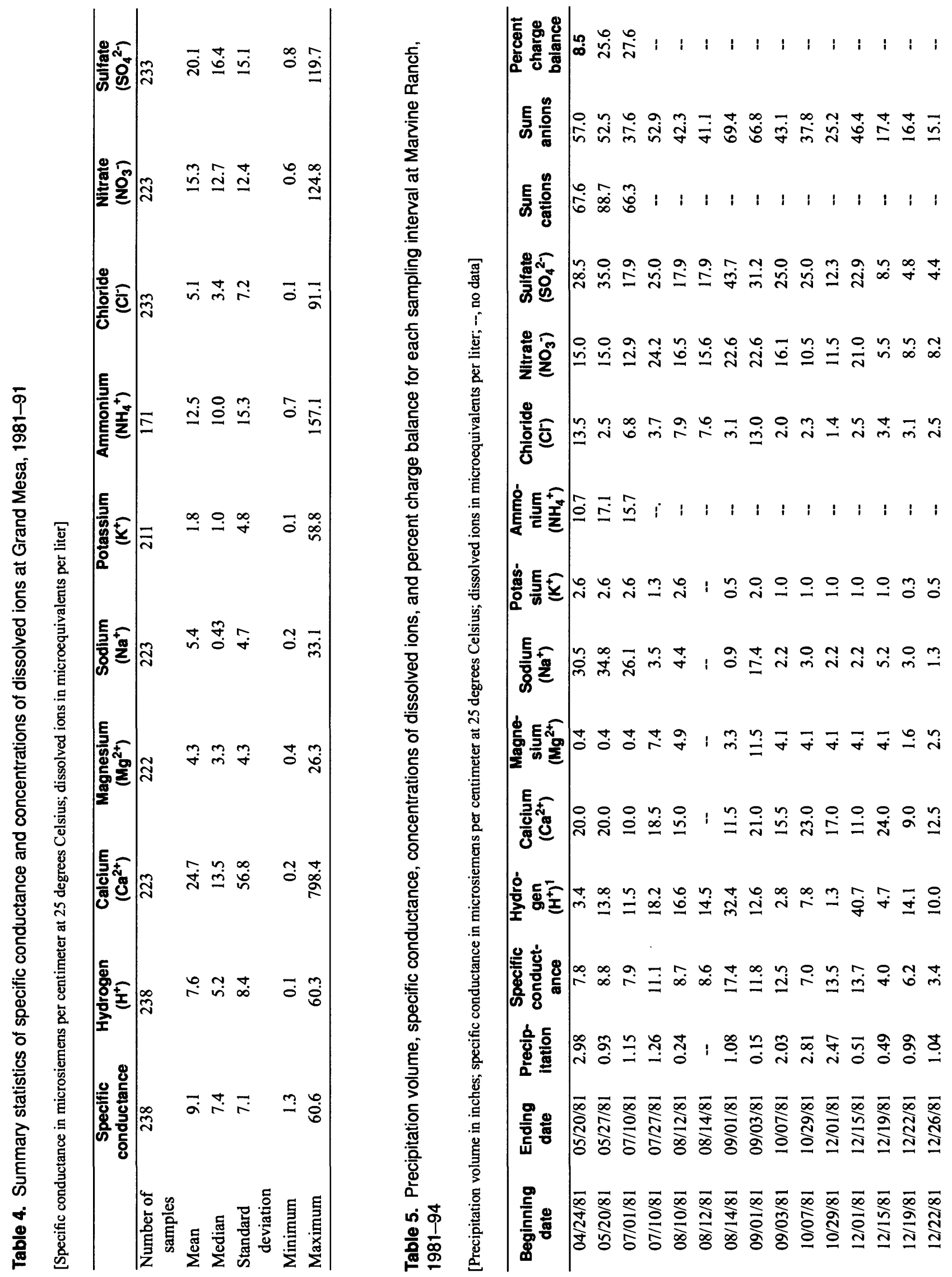




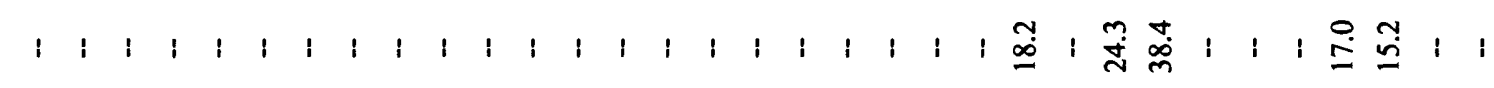

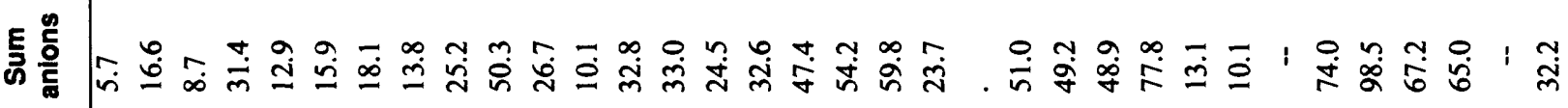

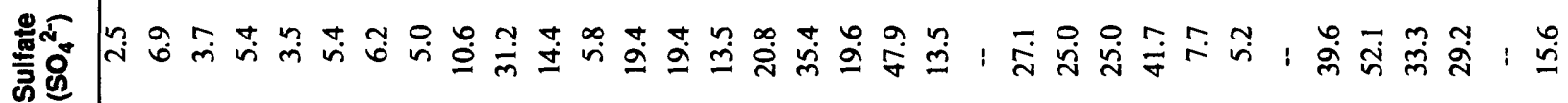

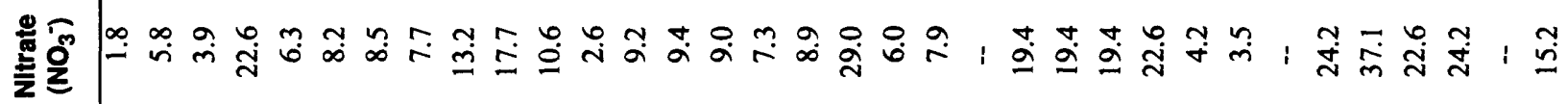

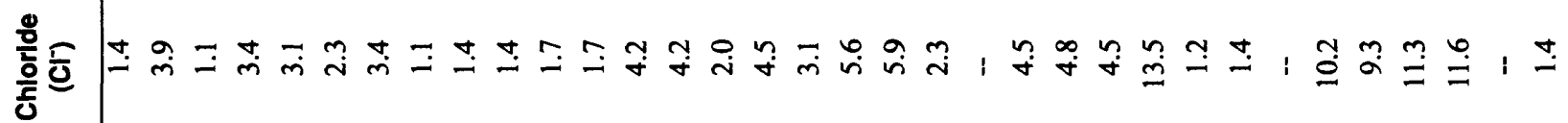

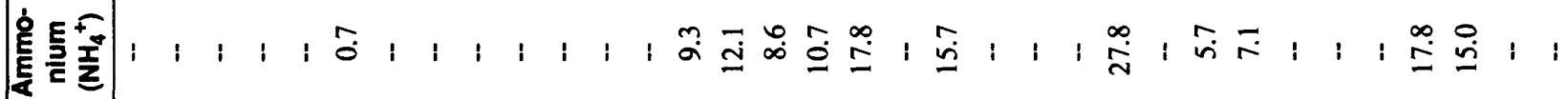

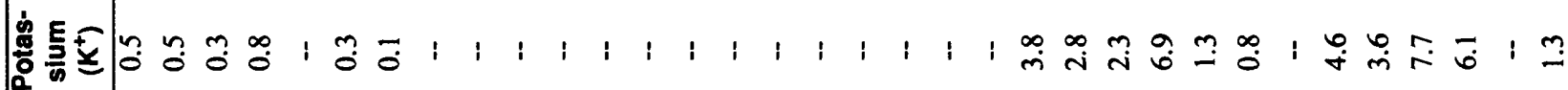
吾尔

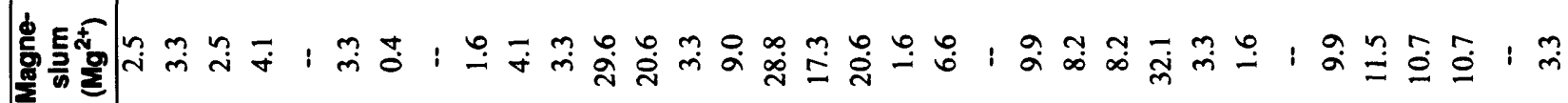
埡热

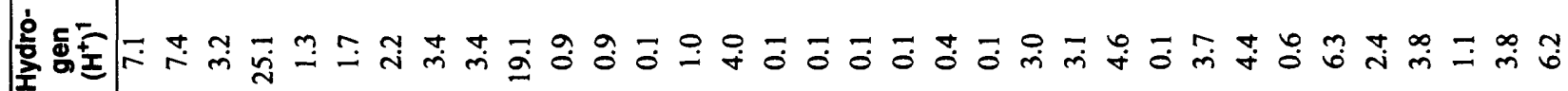

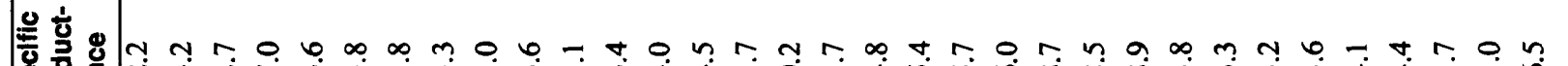

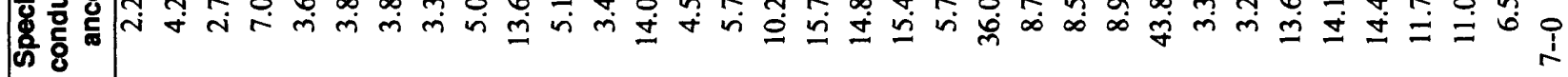

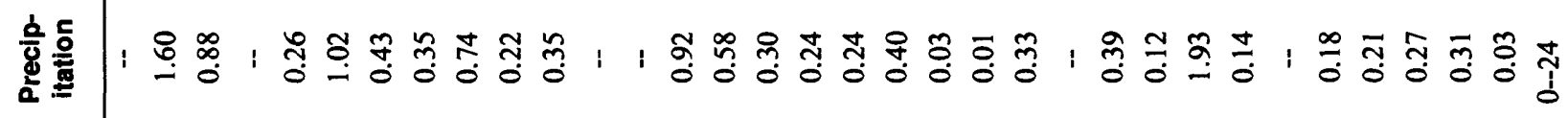

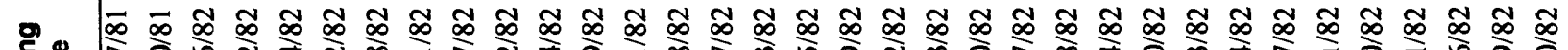
듬

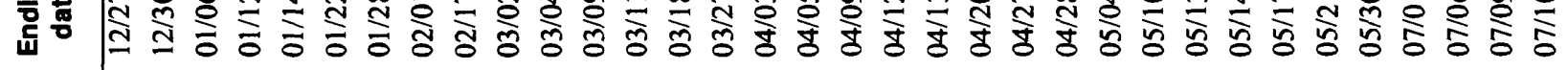

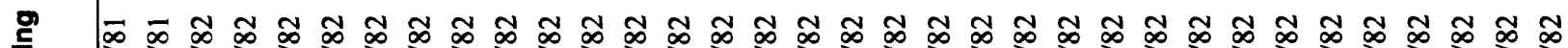
喜密

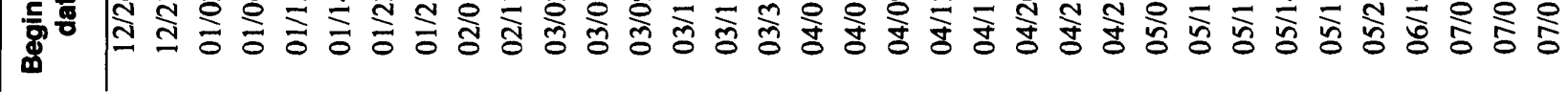




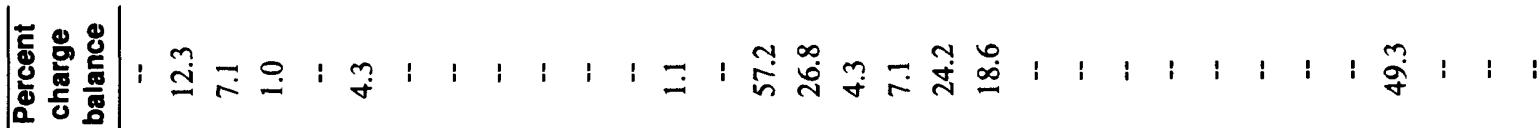

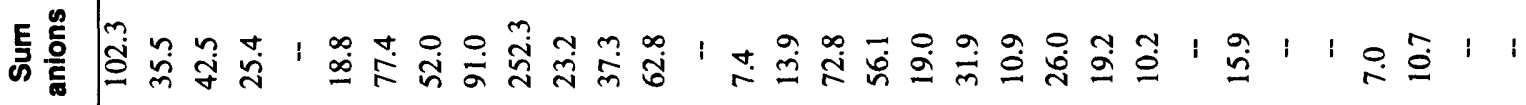

ह

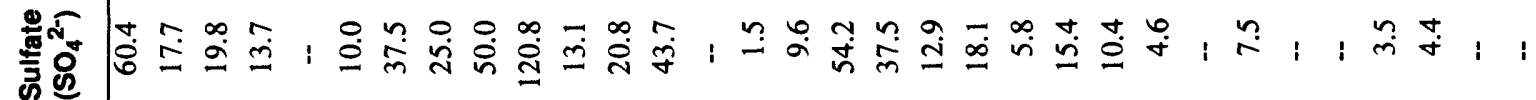

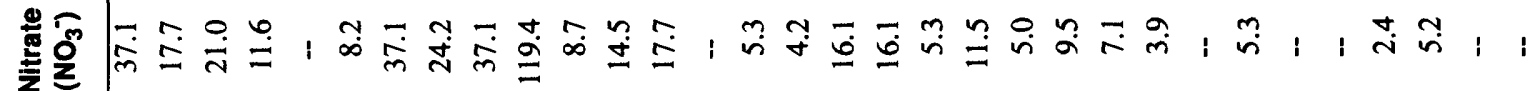

흘잉

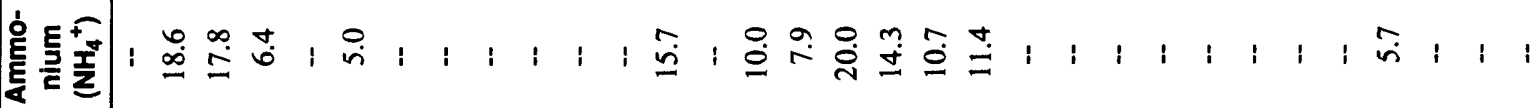

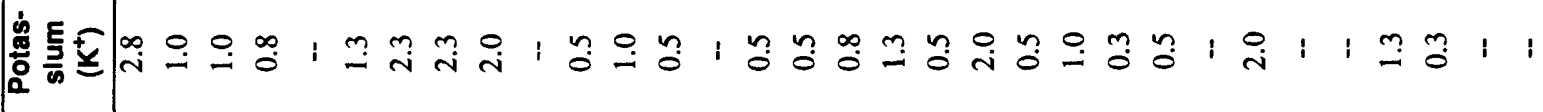

言每

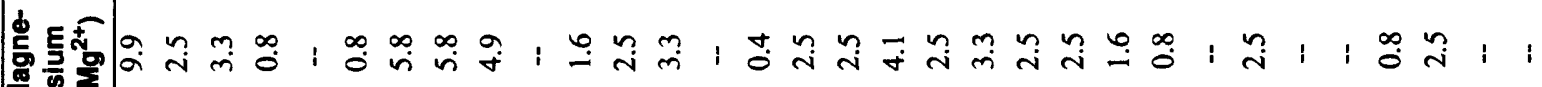

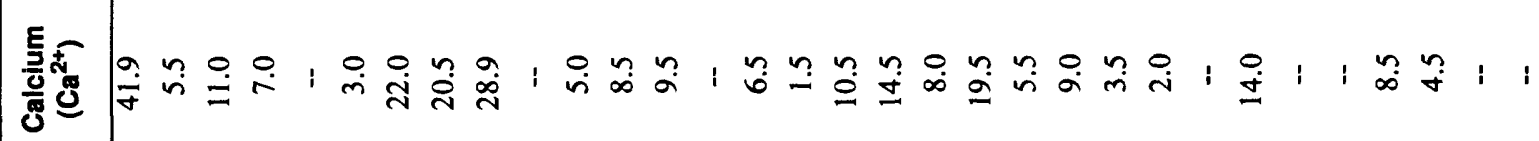

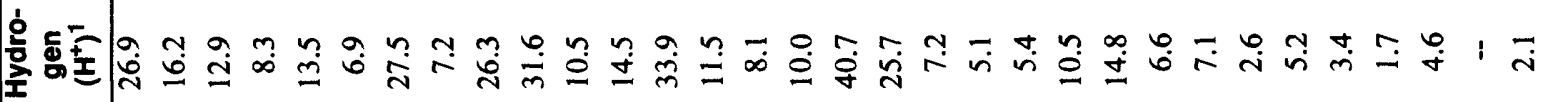

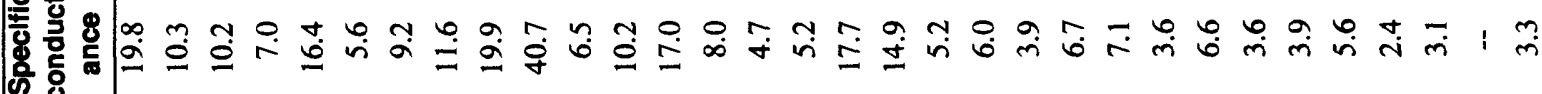

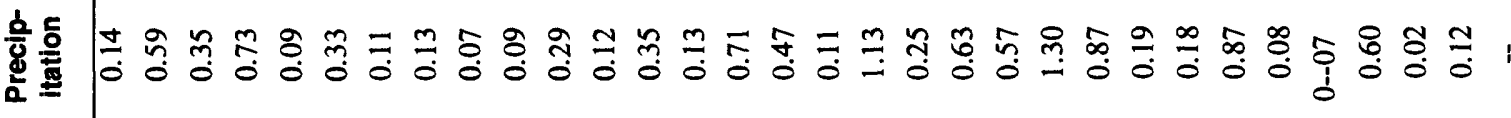

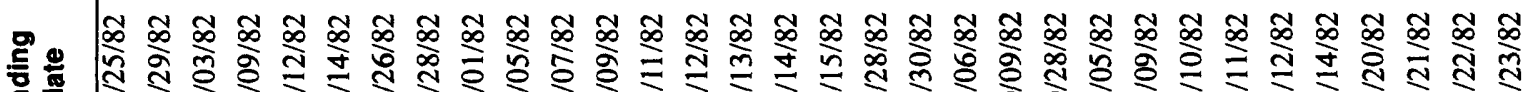

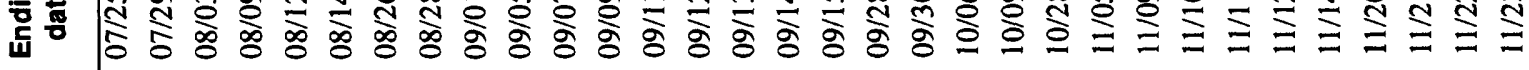

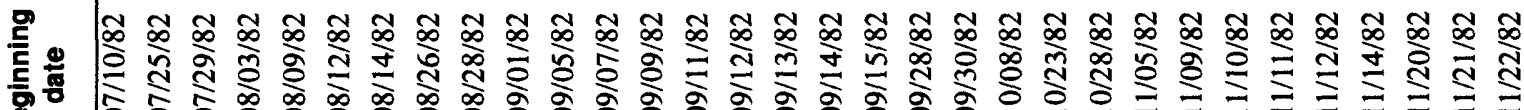
卷 


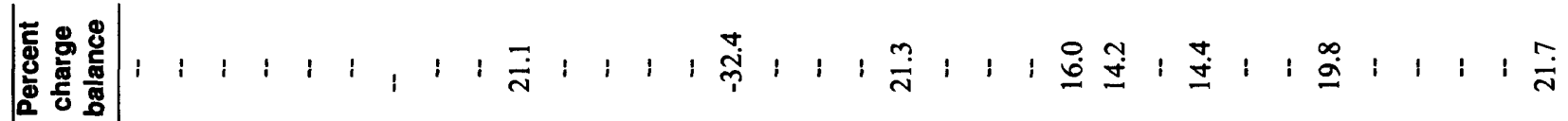

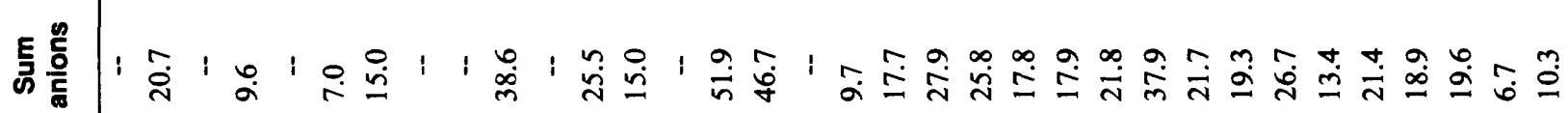

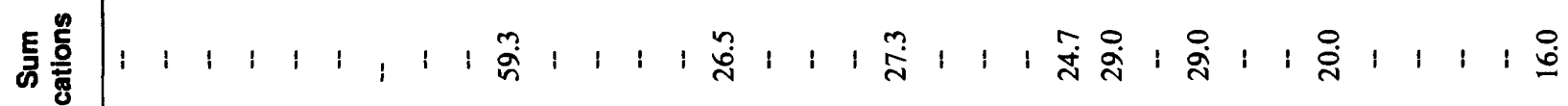
产㑒

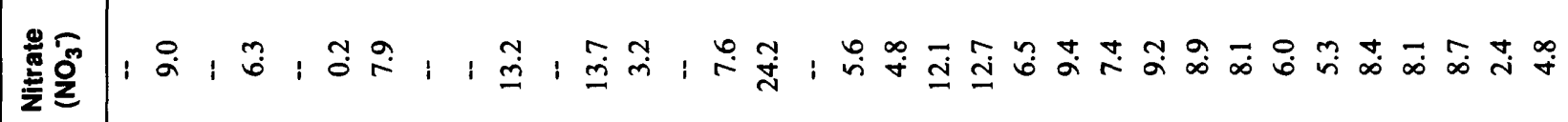

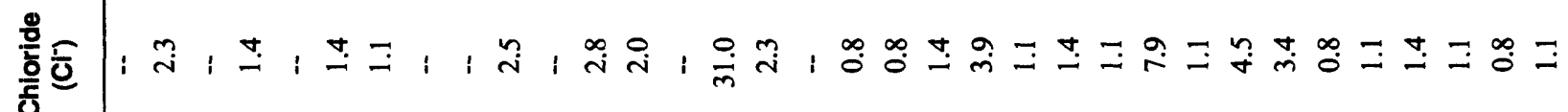

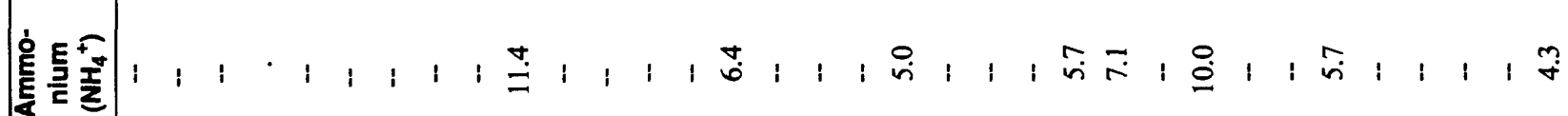

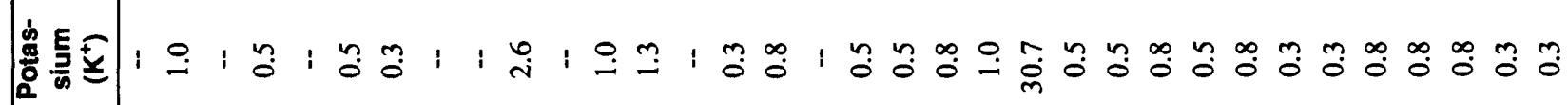
言称

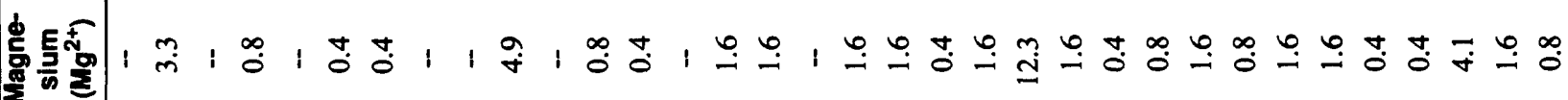

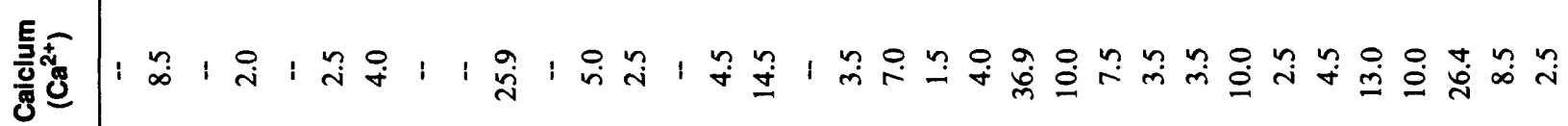

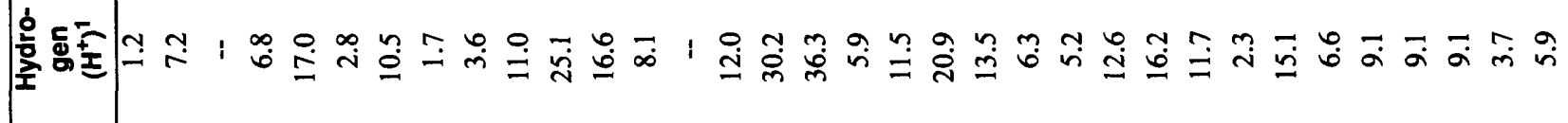

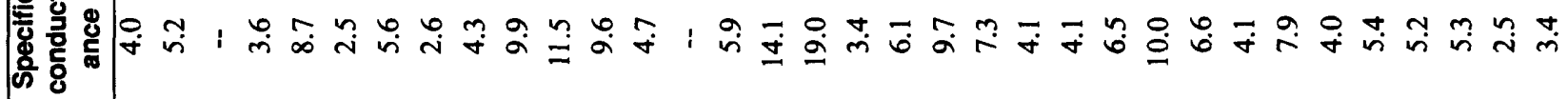

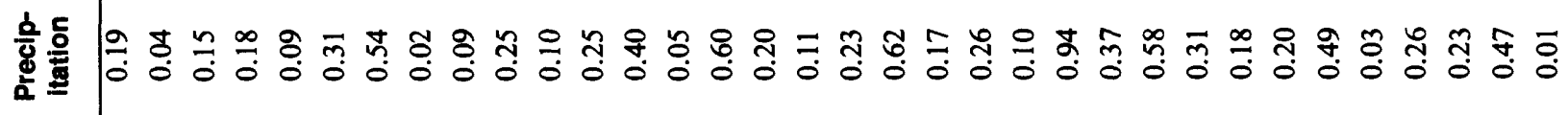

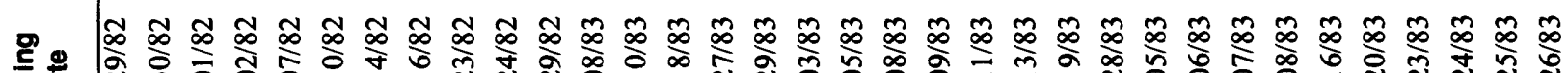

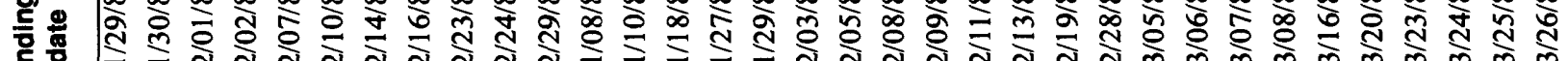

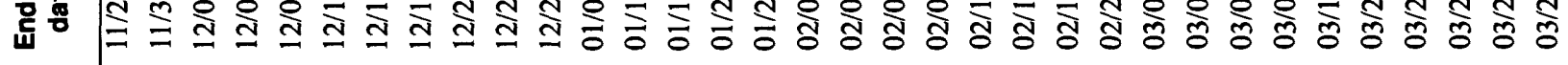

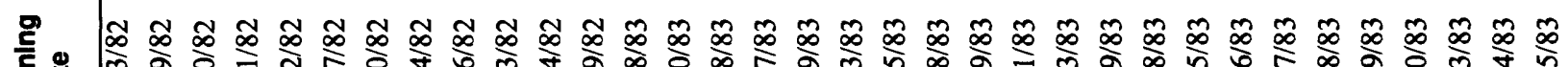
㾍嵒 


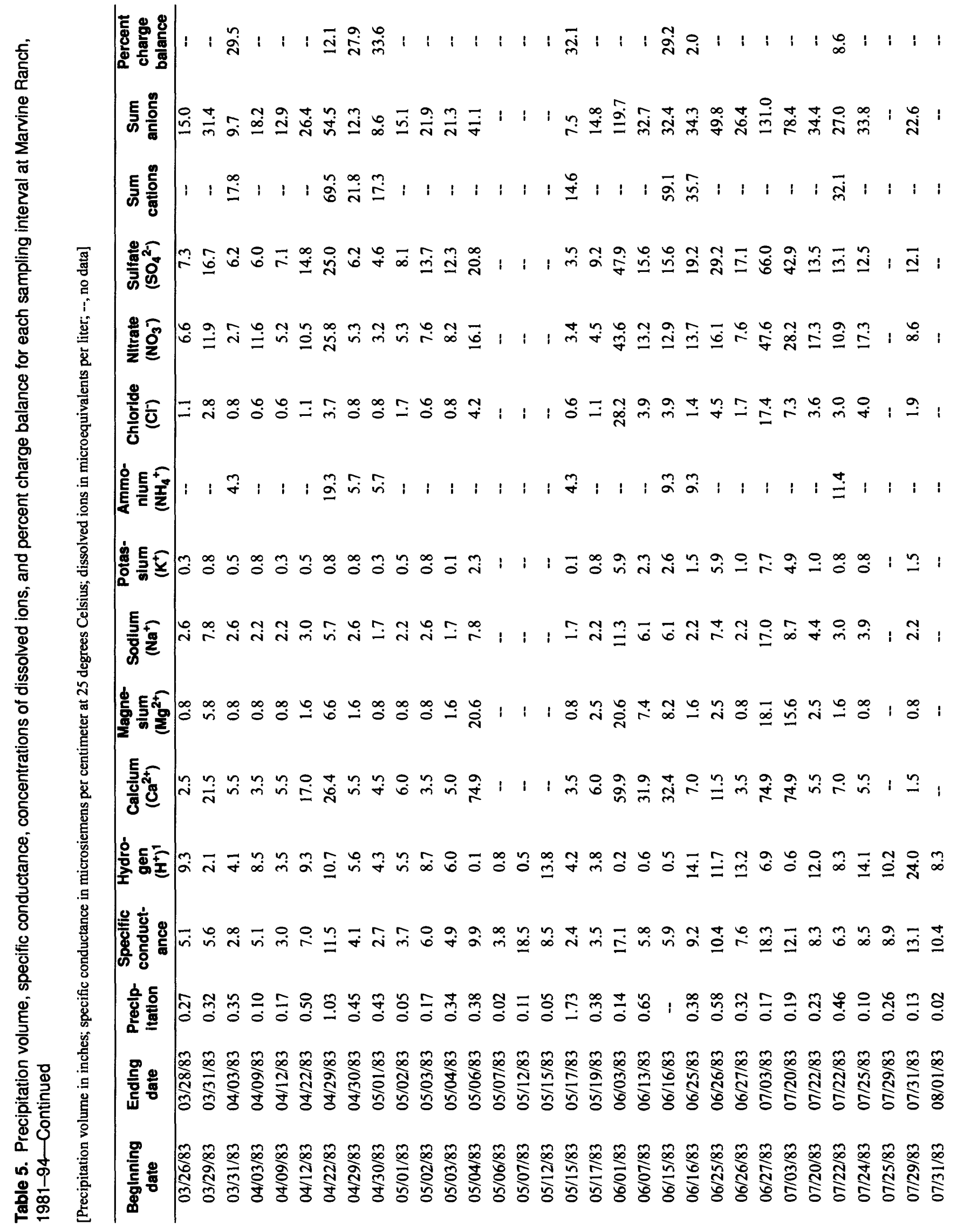




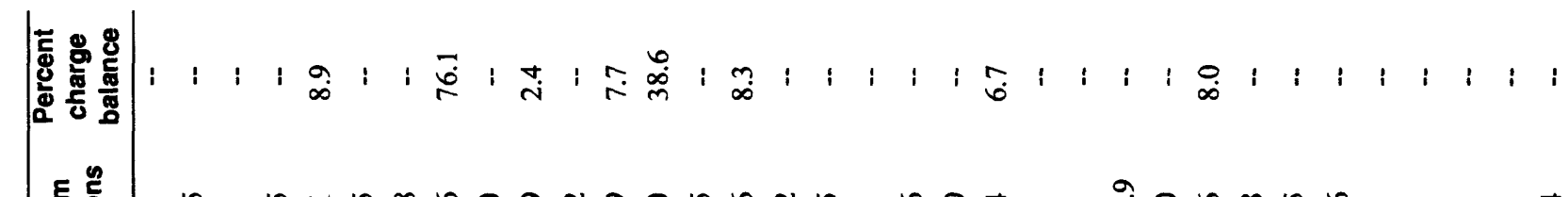

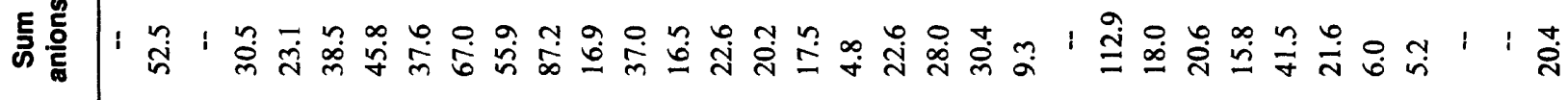

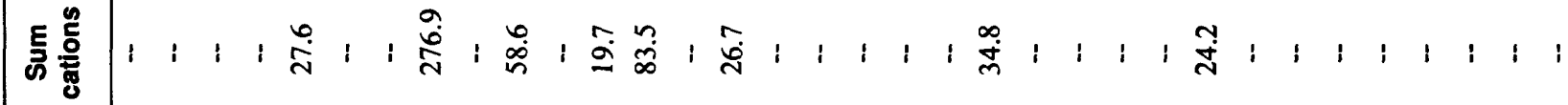
क

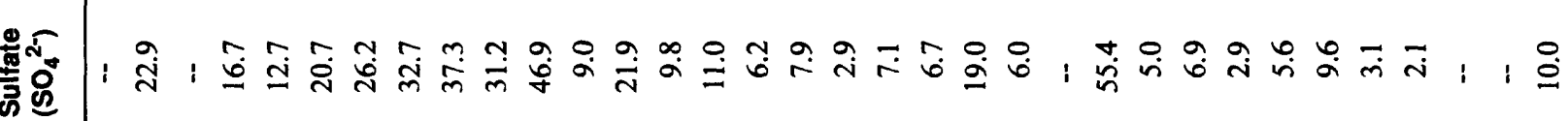

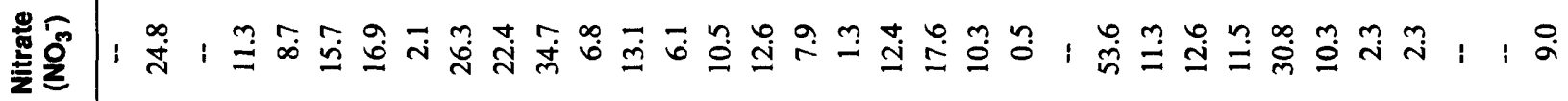
흘잉

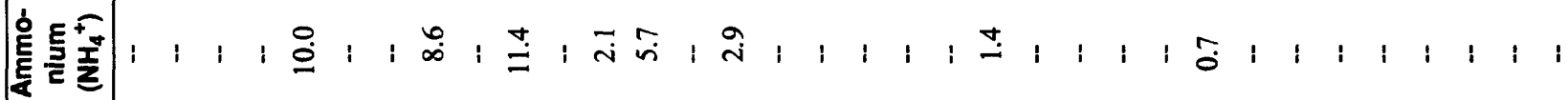

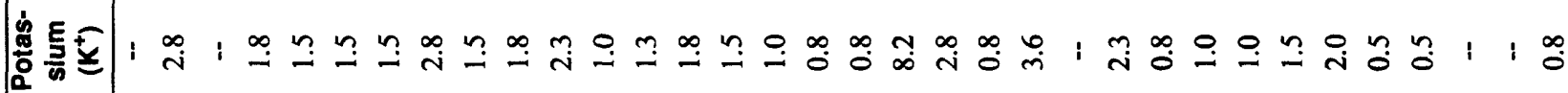

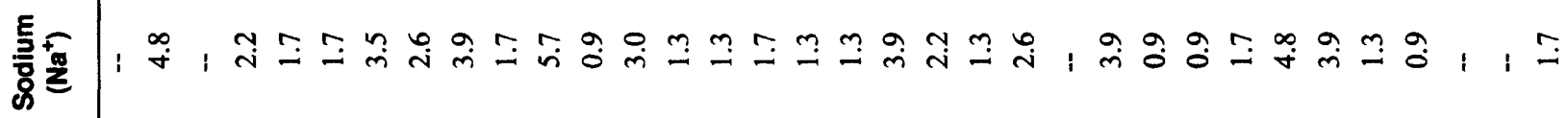

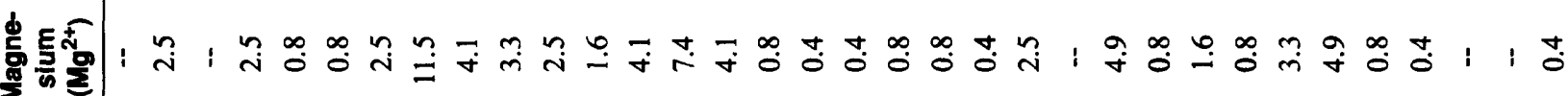

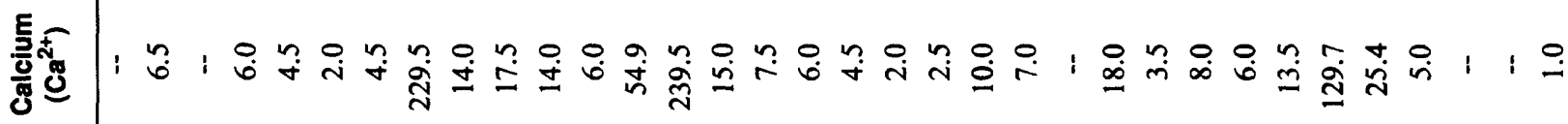

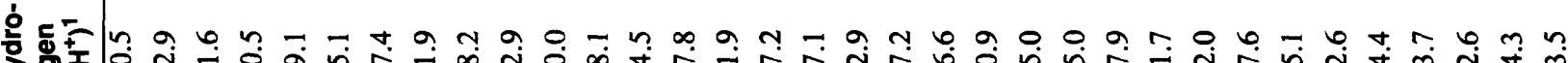

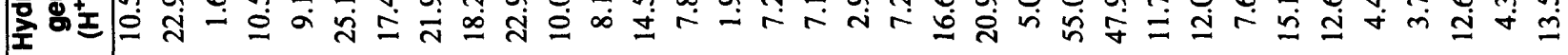
는

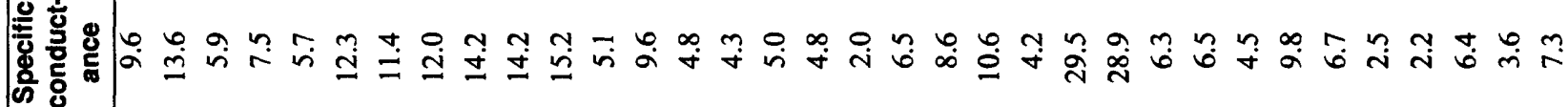
흔흘

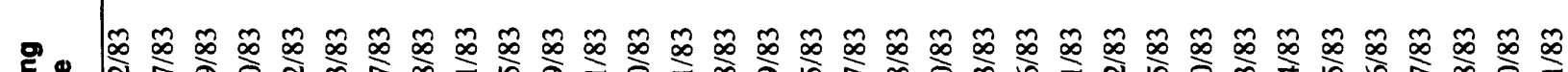
흠 w.

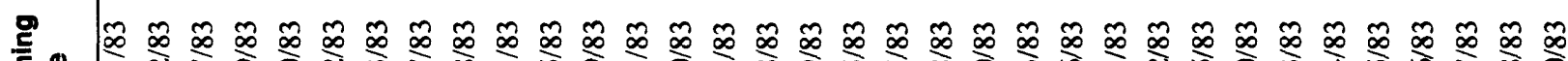
든 कo 


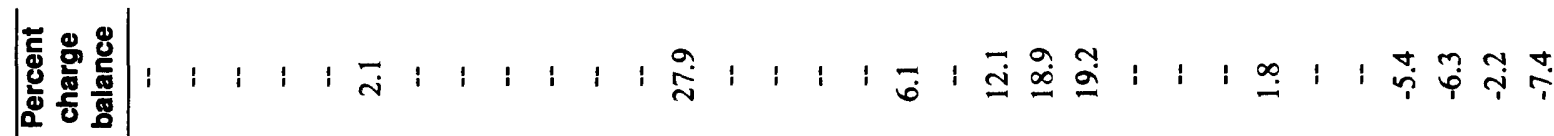

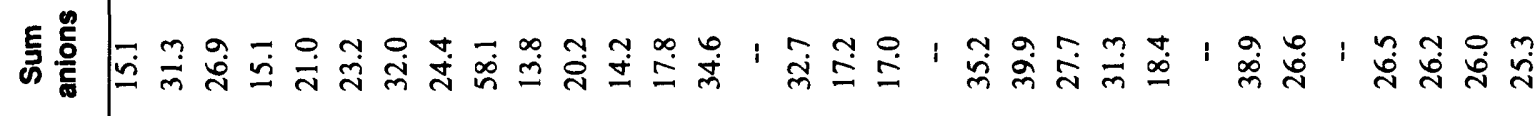
ह气

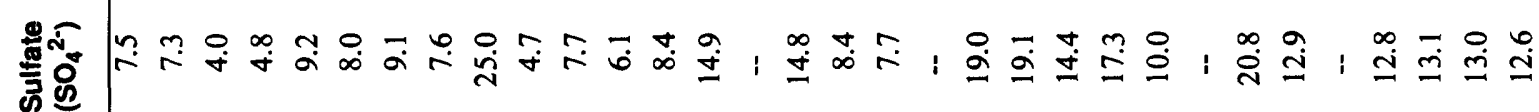

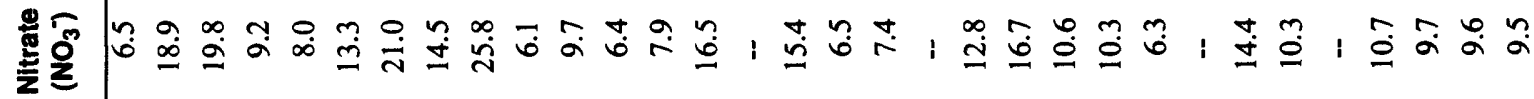

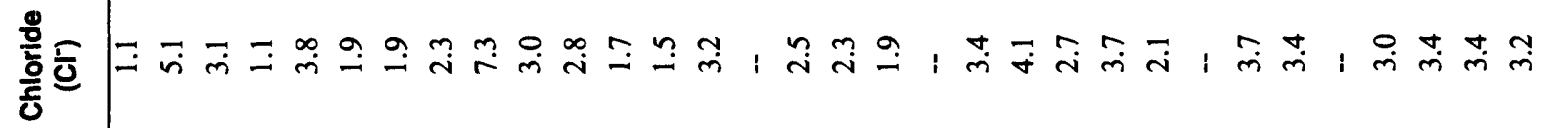

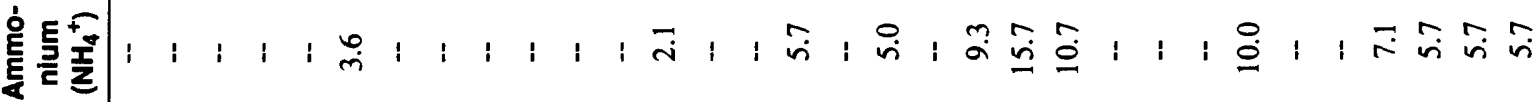

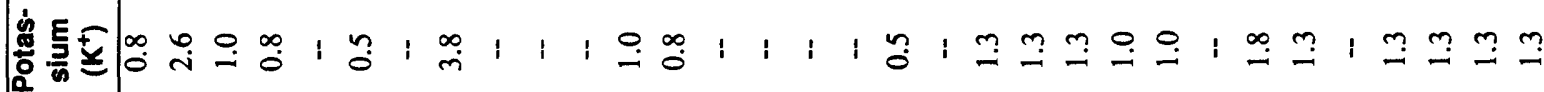

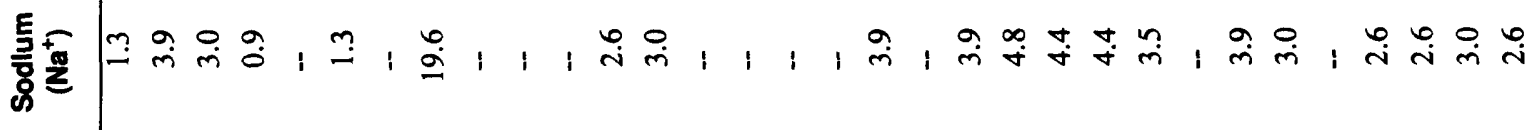

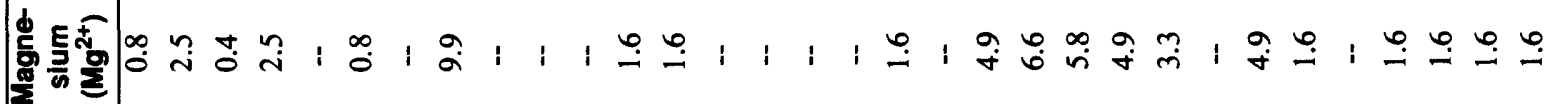

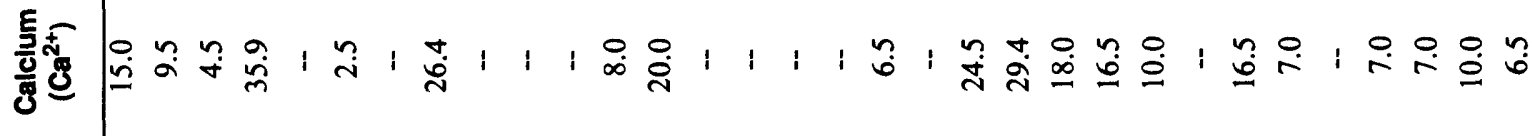

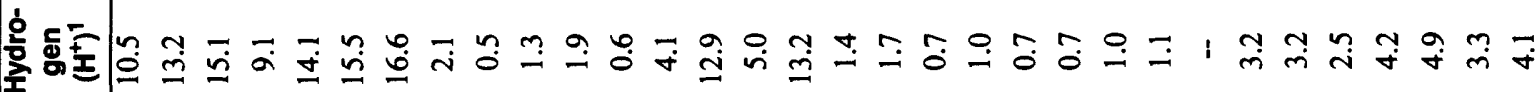
$0 \stackrel{4}{\mathrm{U}}$

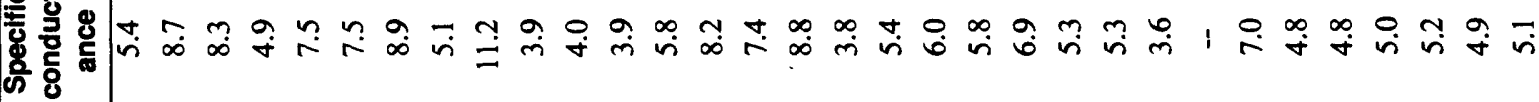

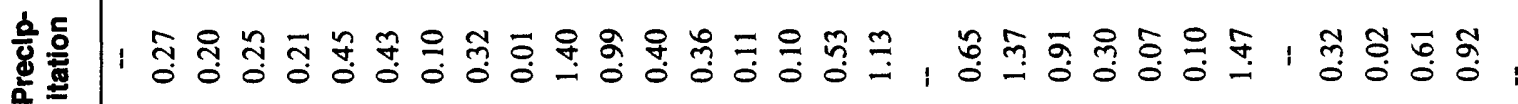
迹

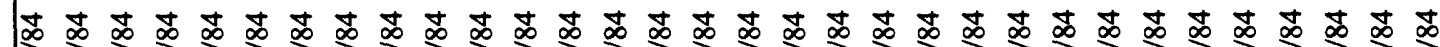

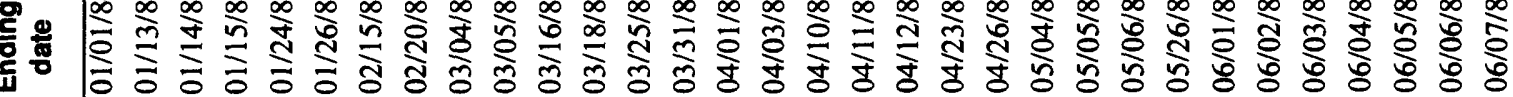

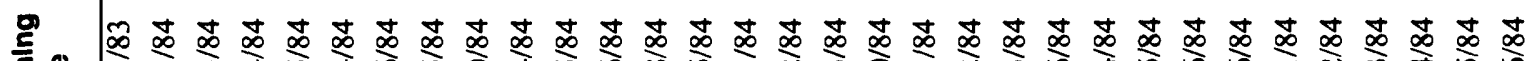
恶 
|

䓞旁 宓旁

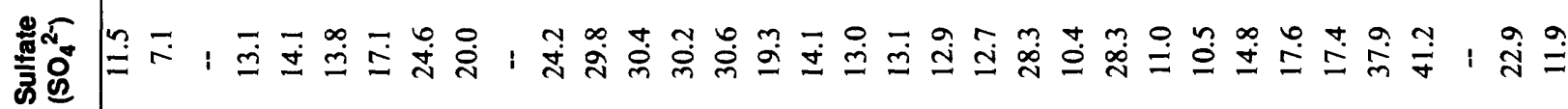

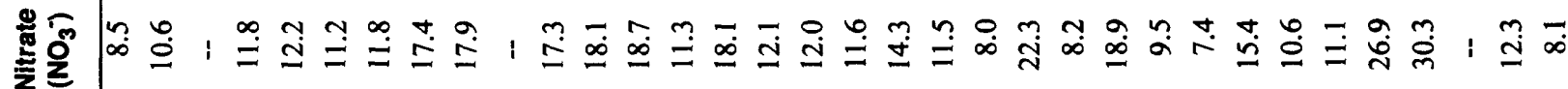

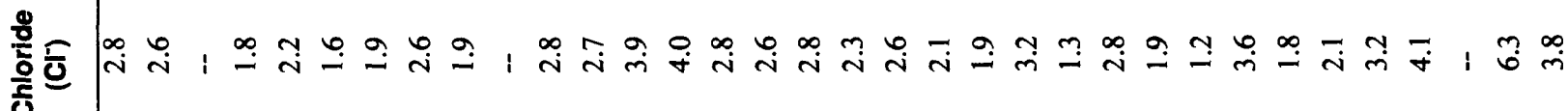

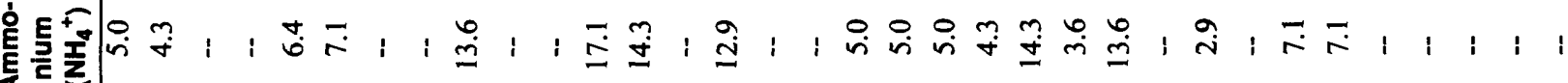

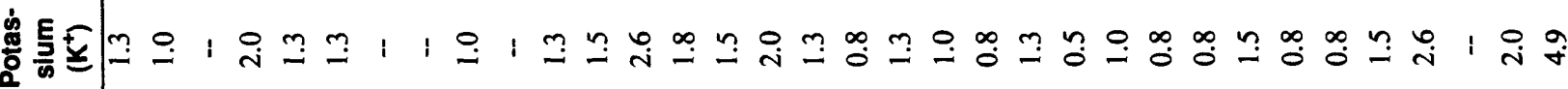

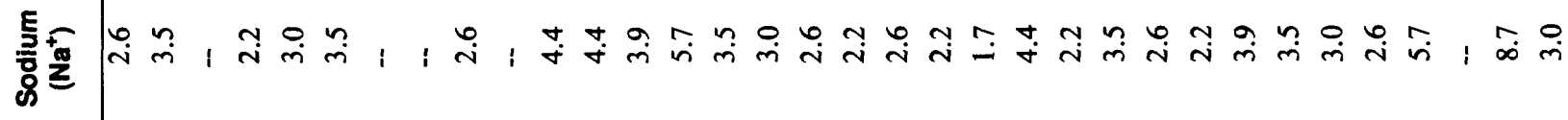

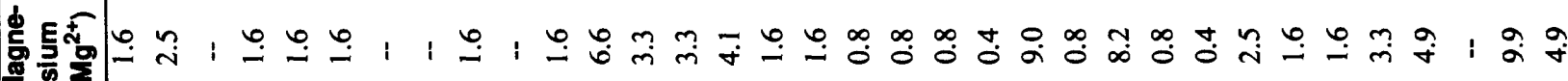

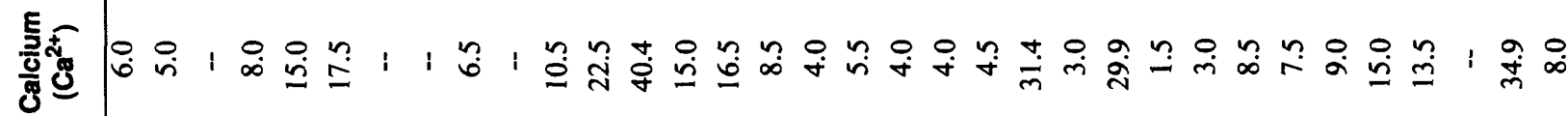

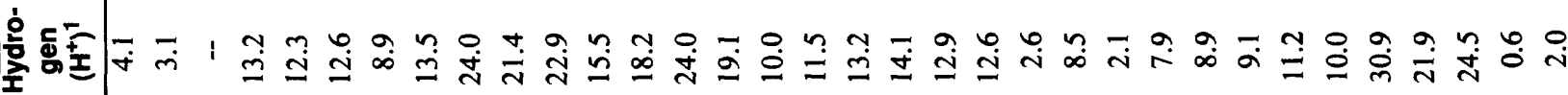
은 它 市兽

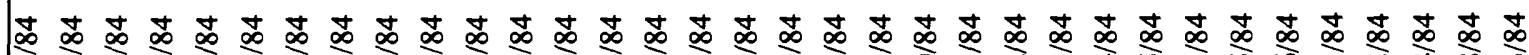
읗

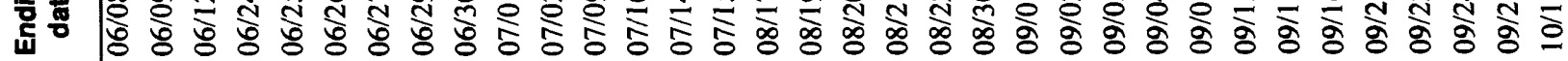

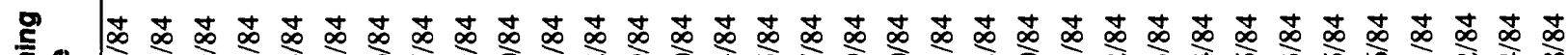

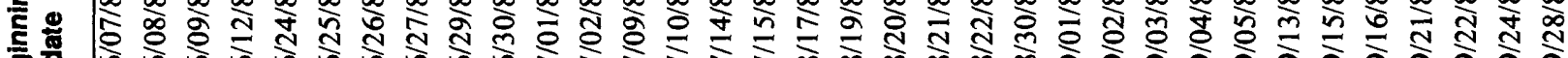
着 


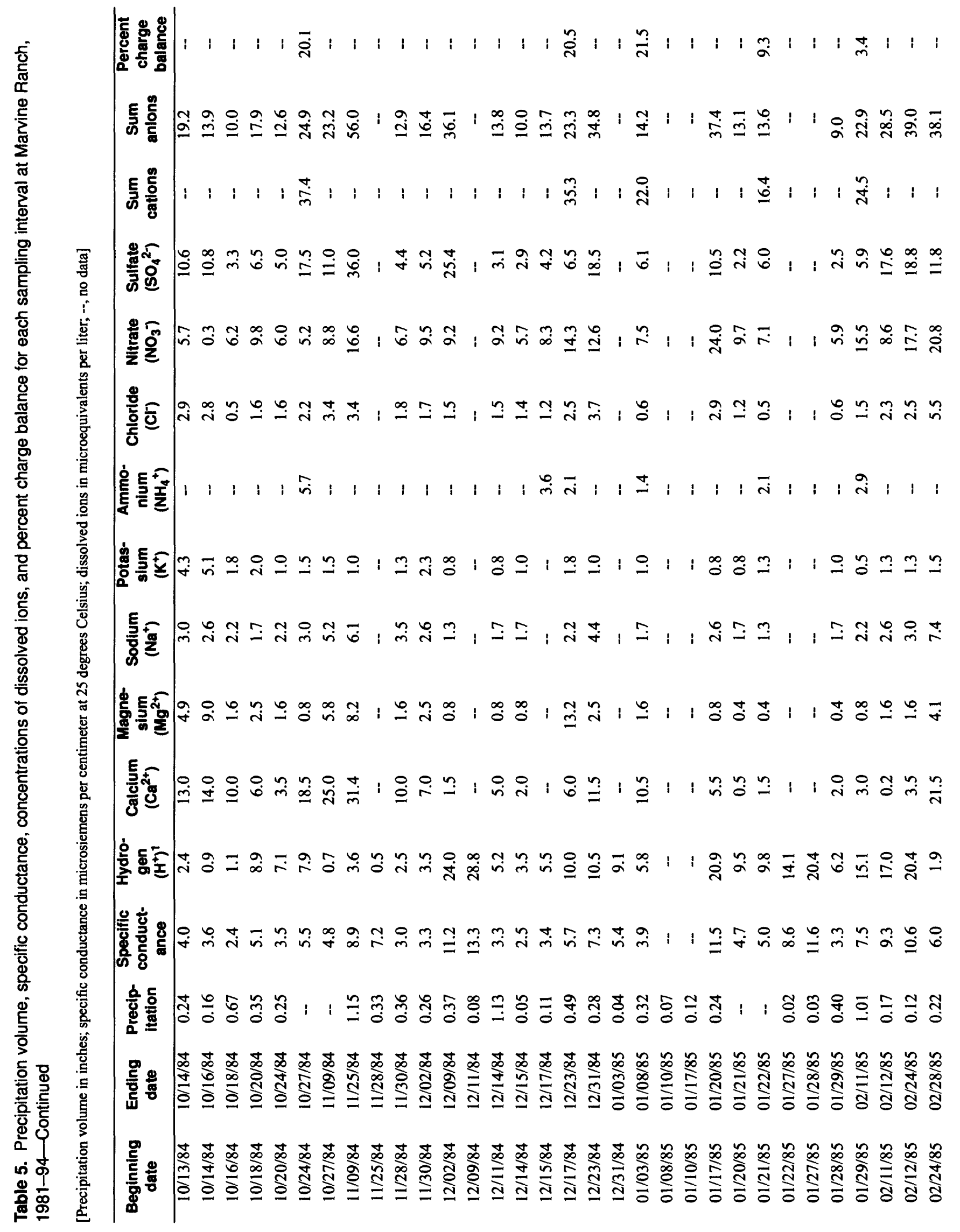




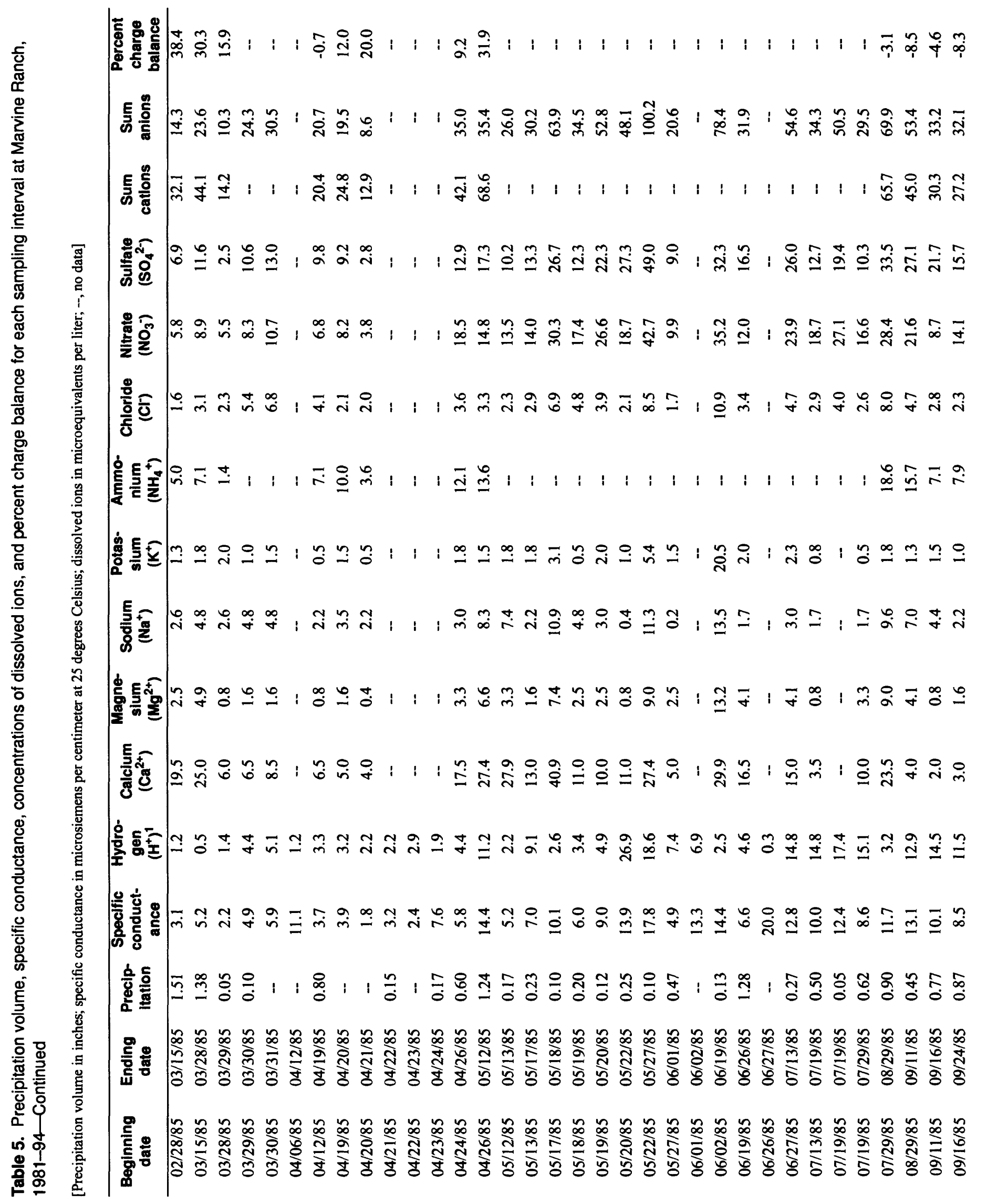




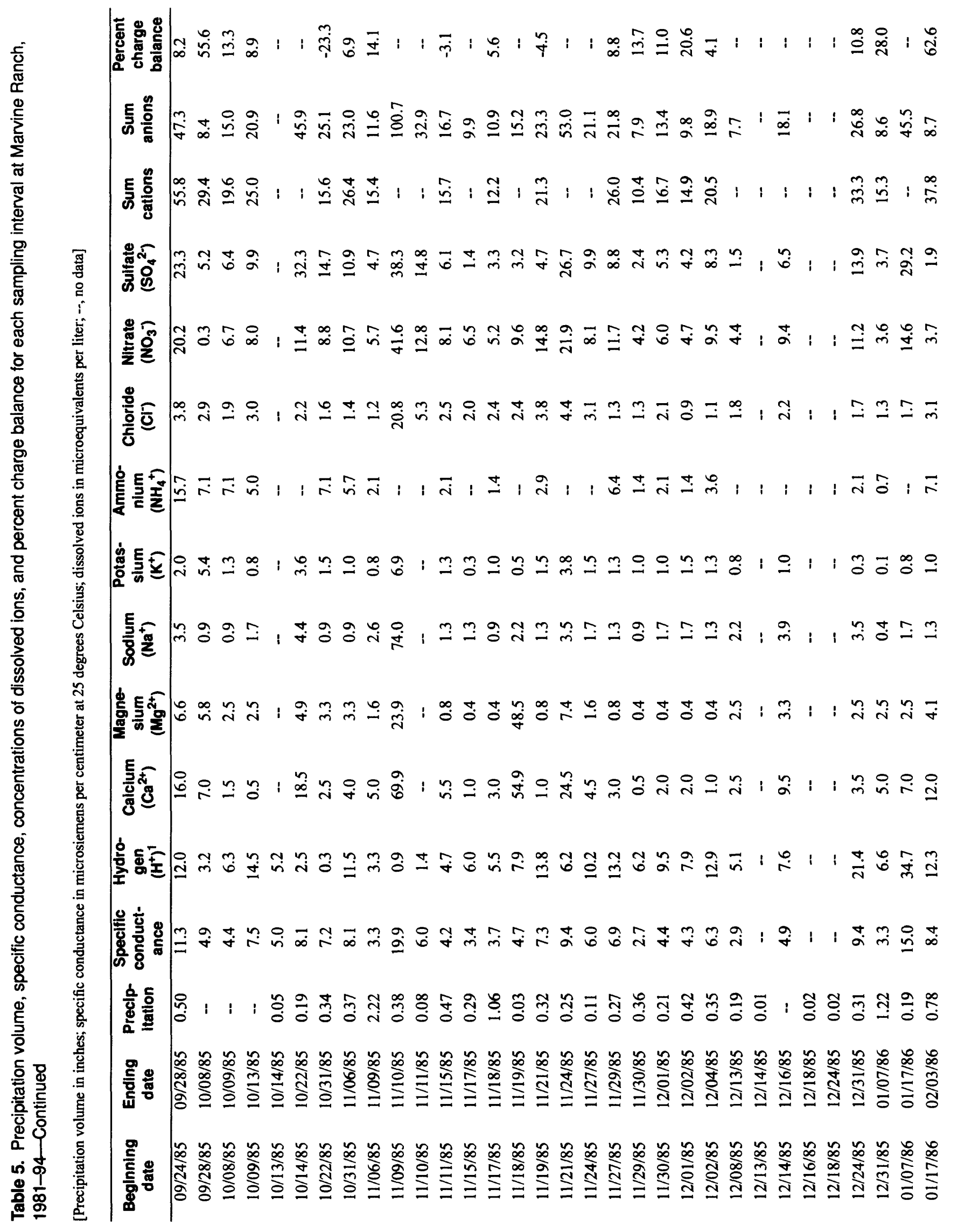




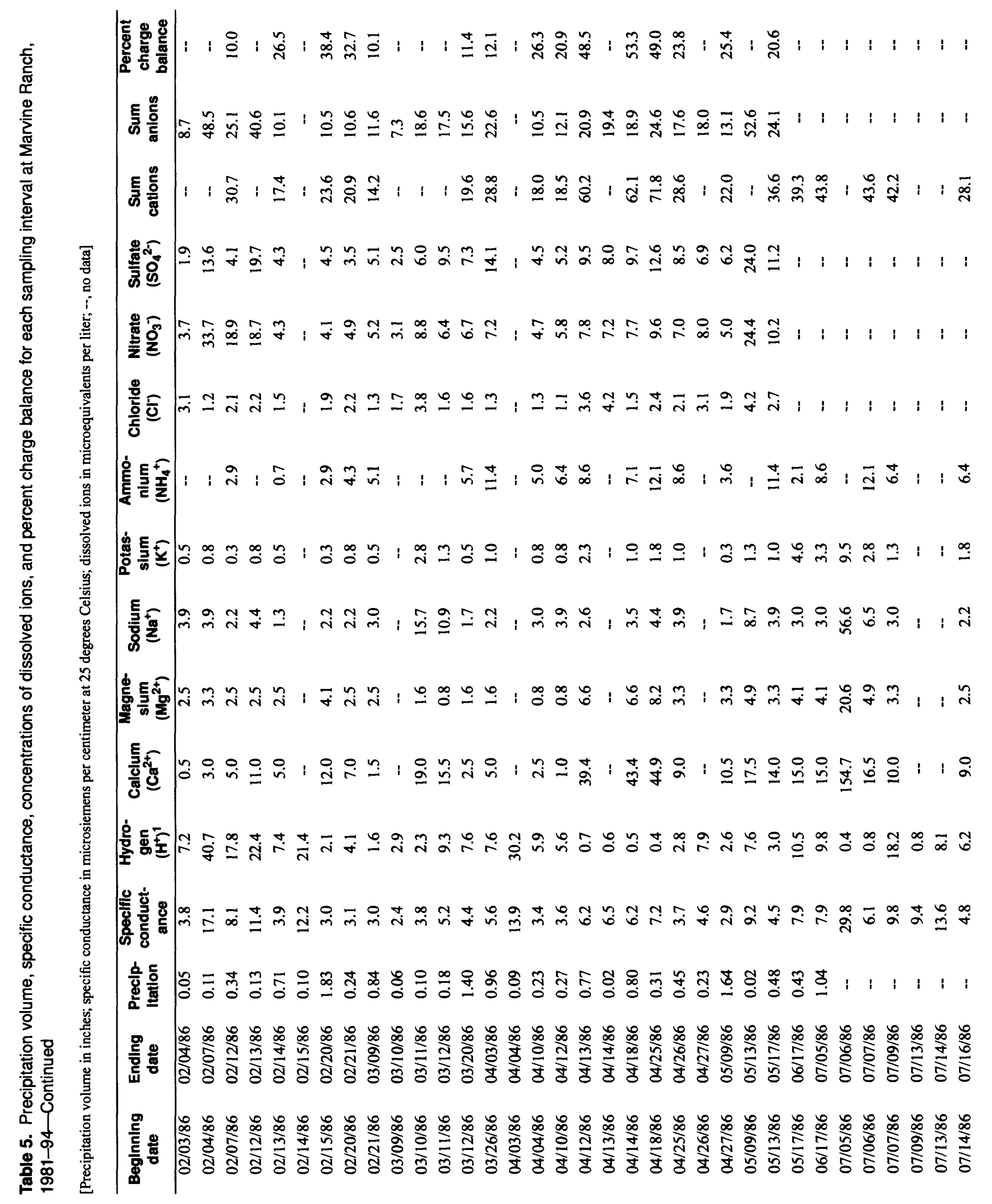




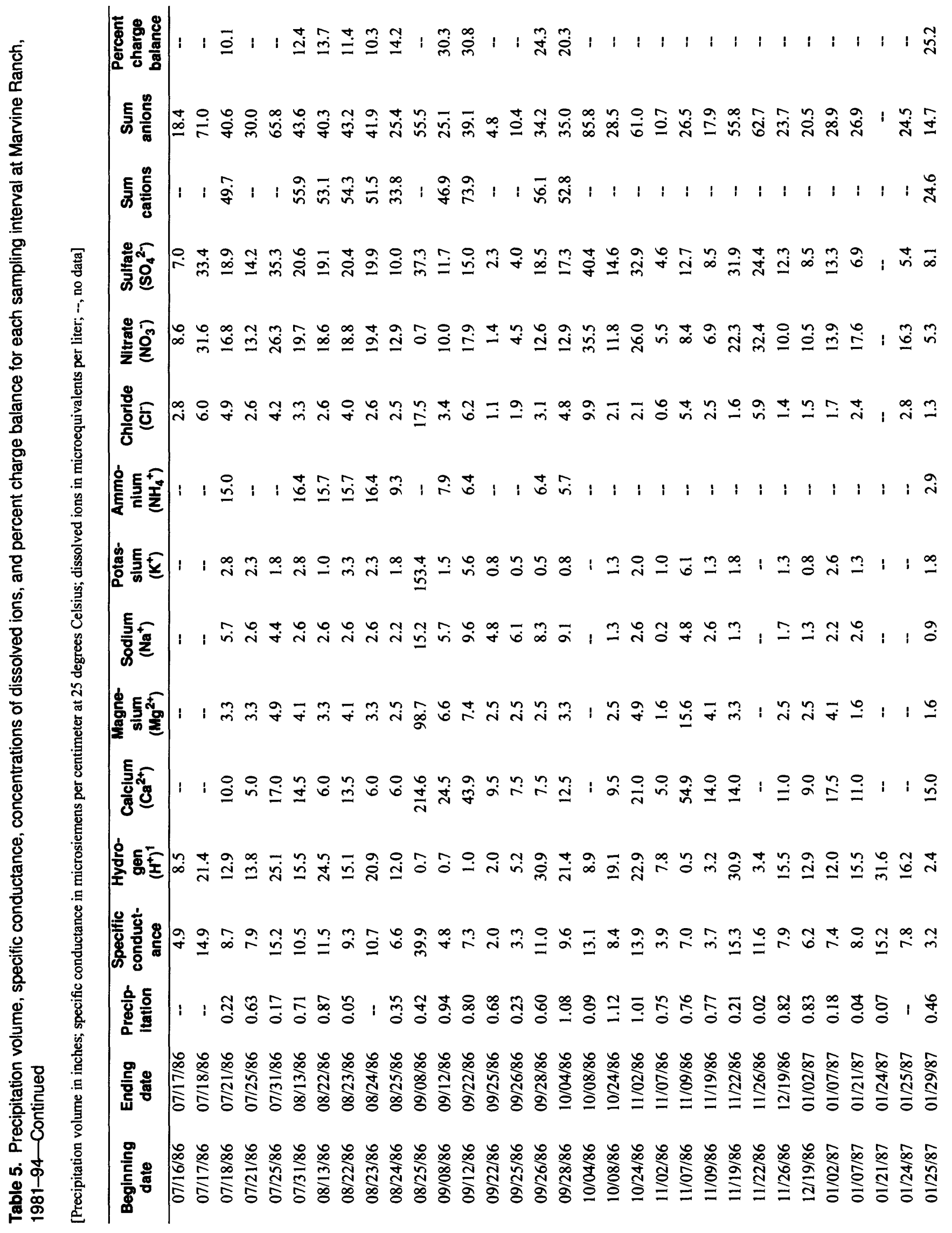




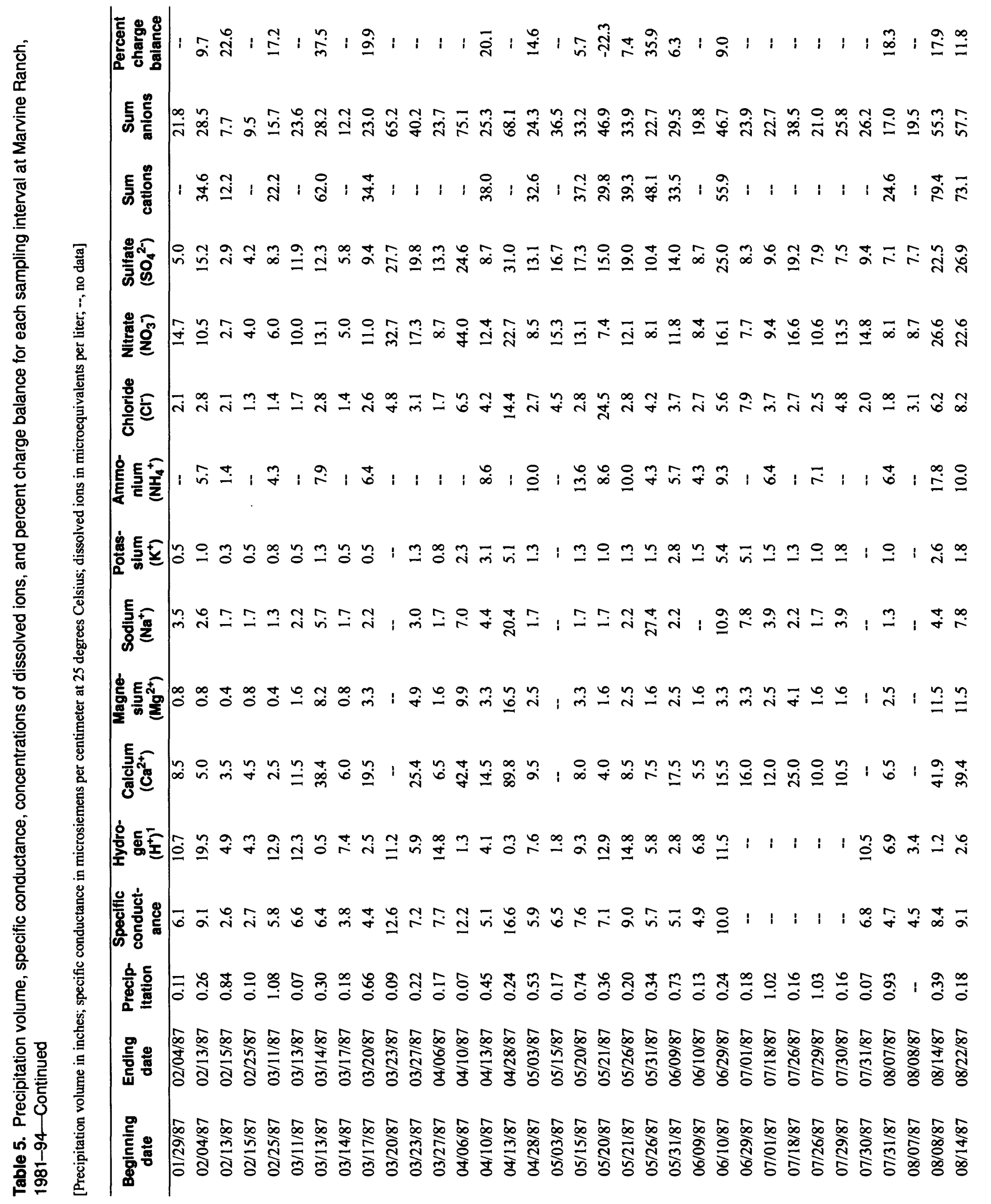




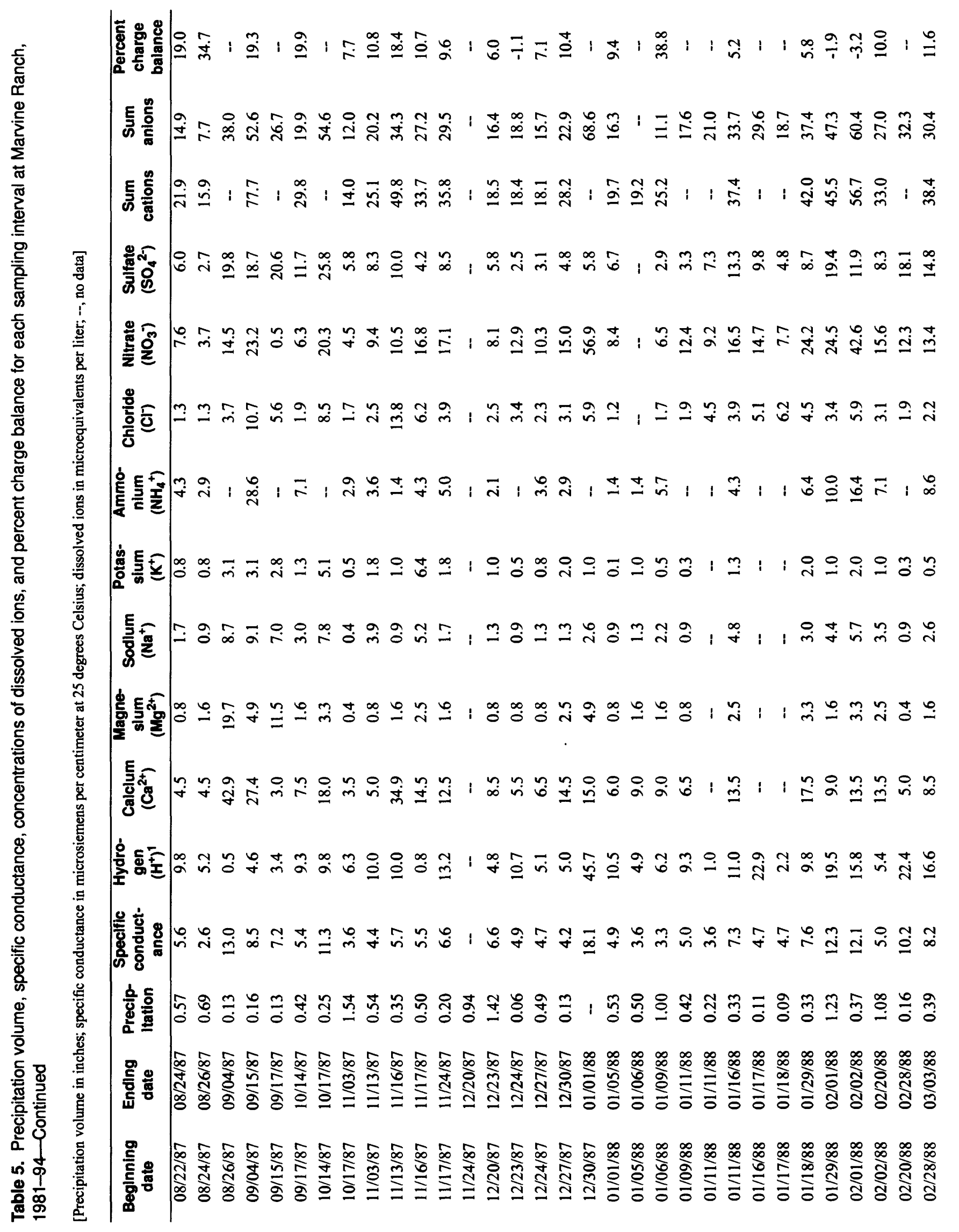




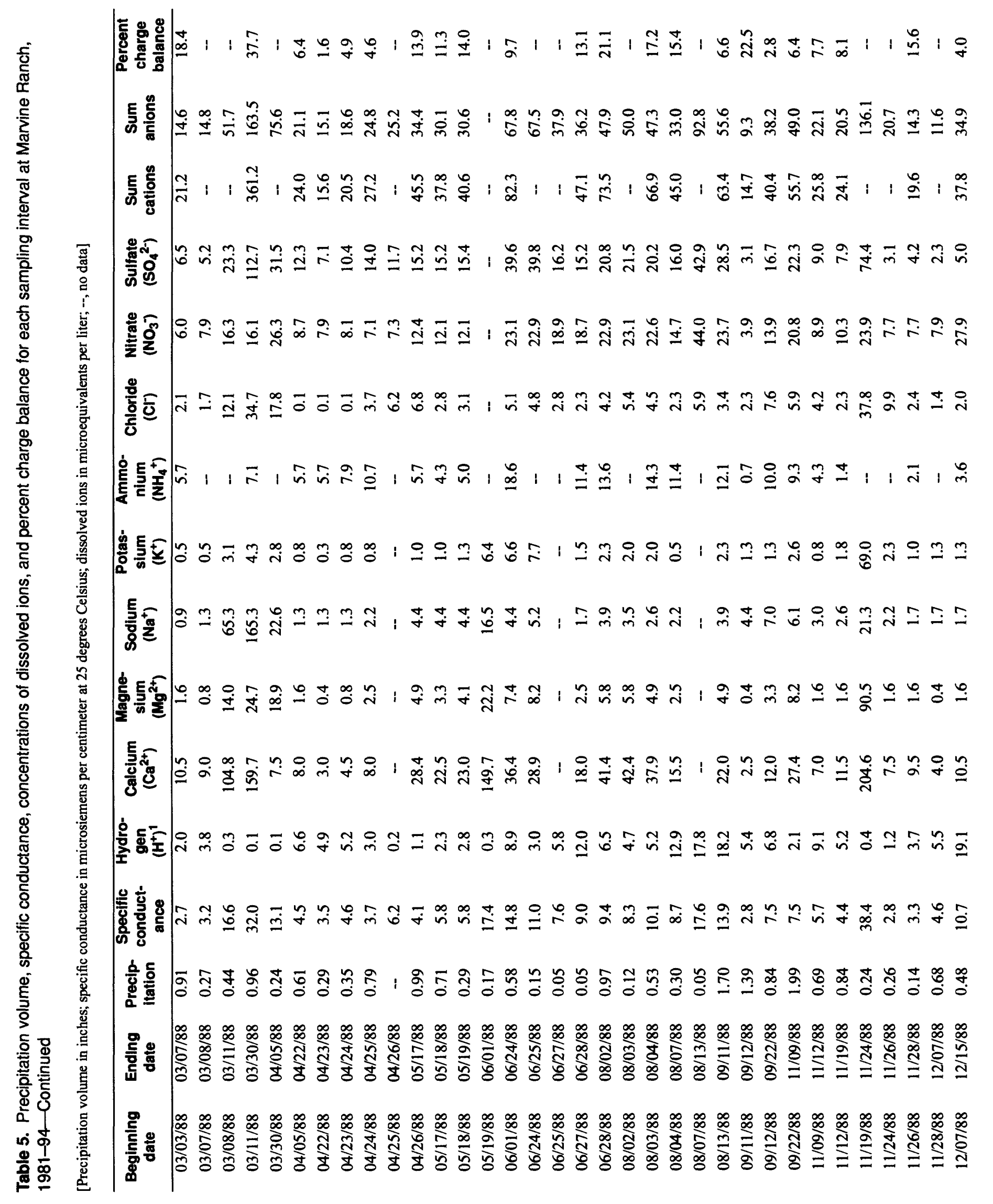




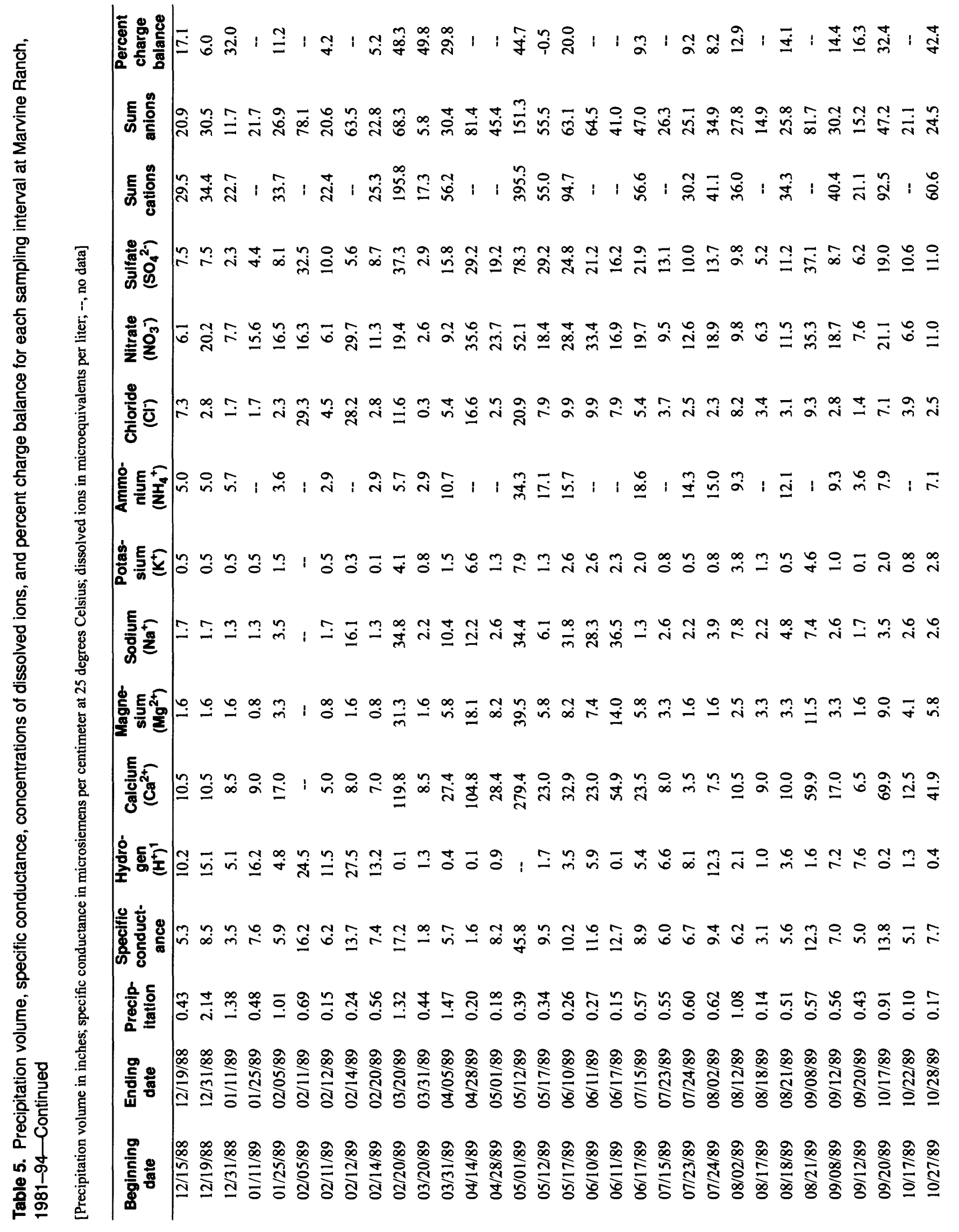




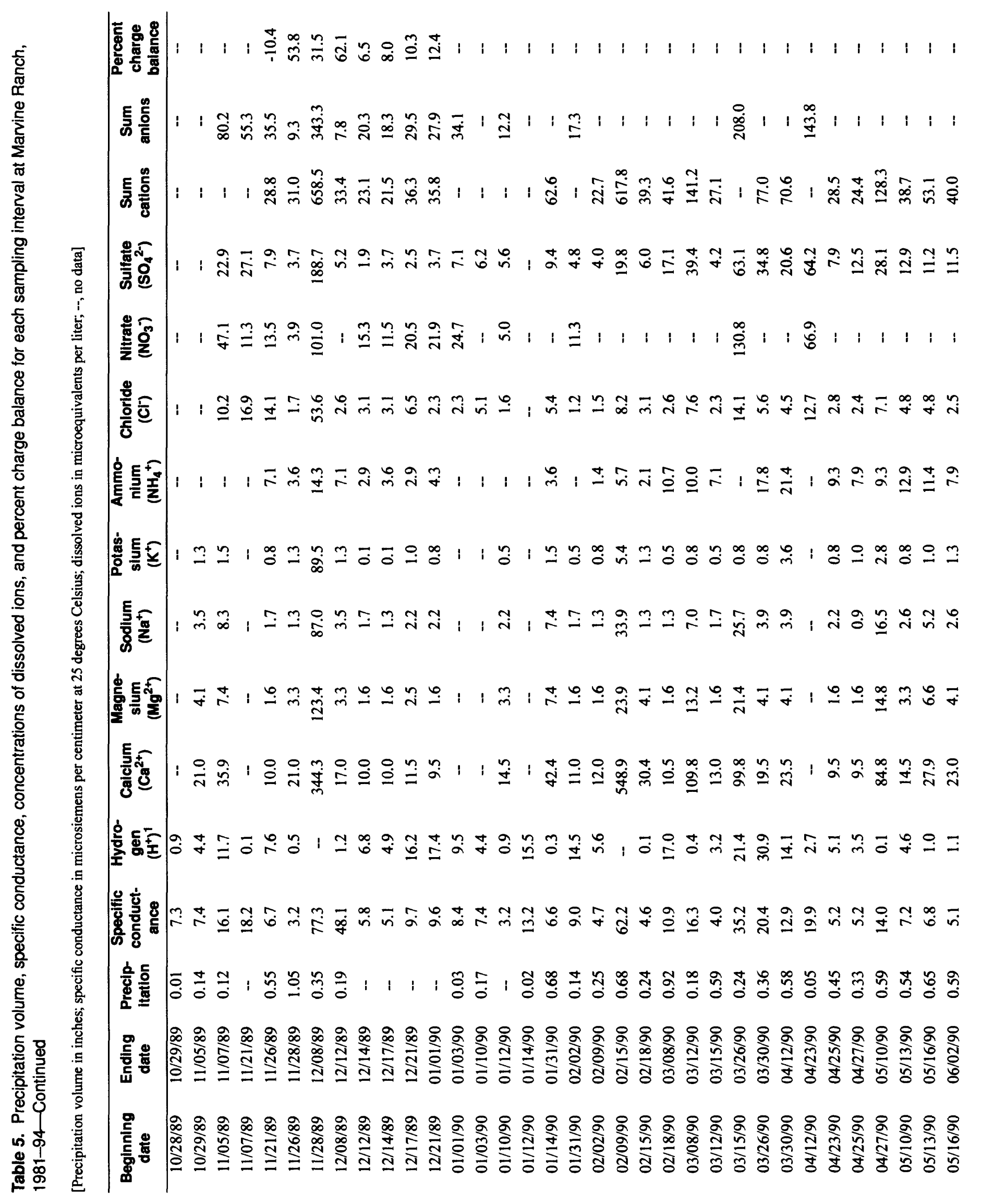




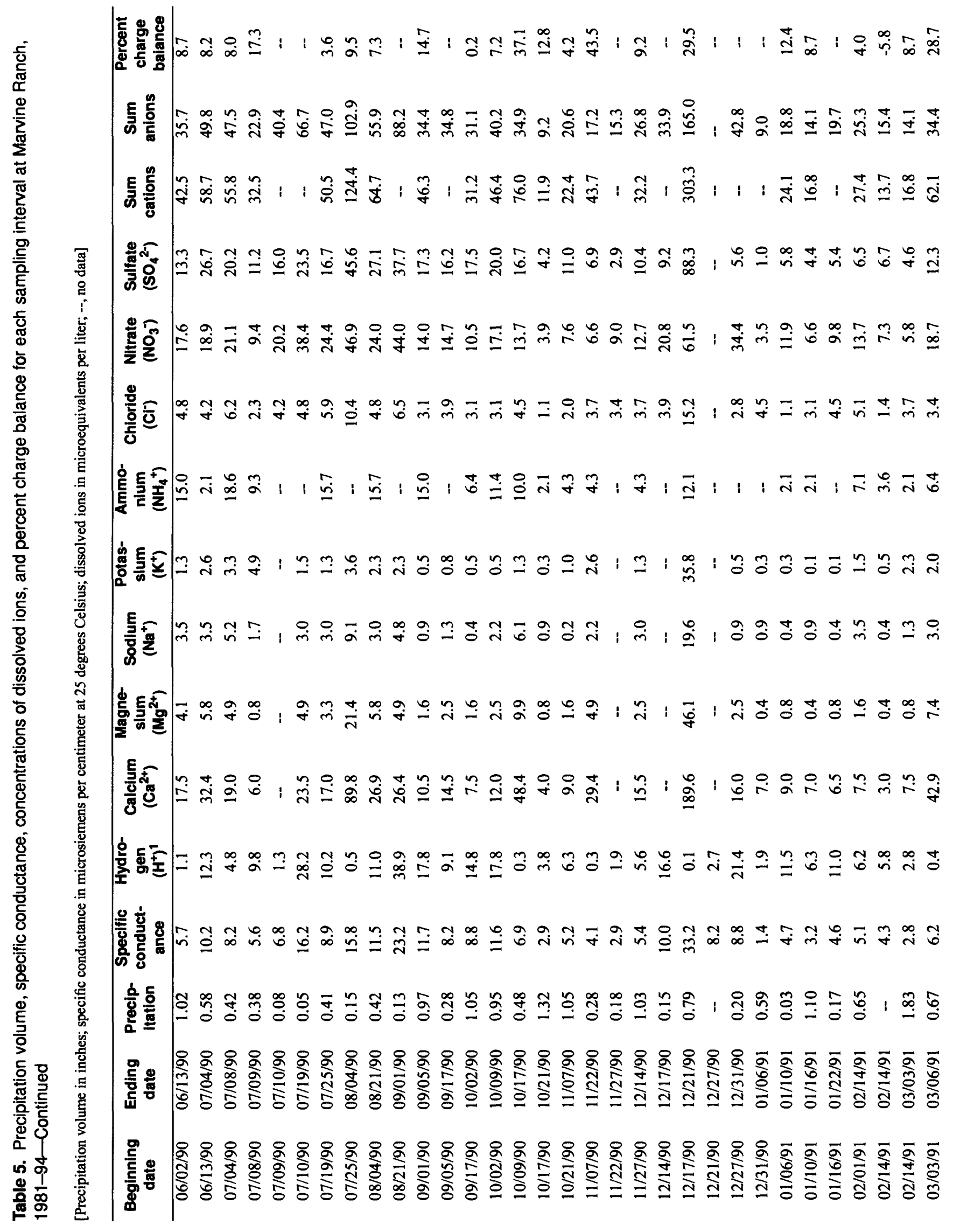




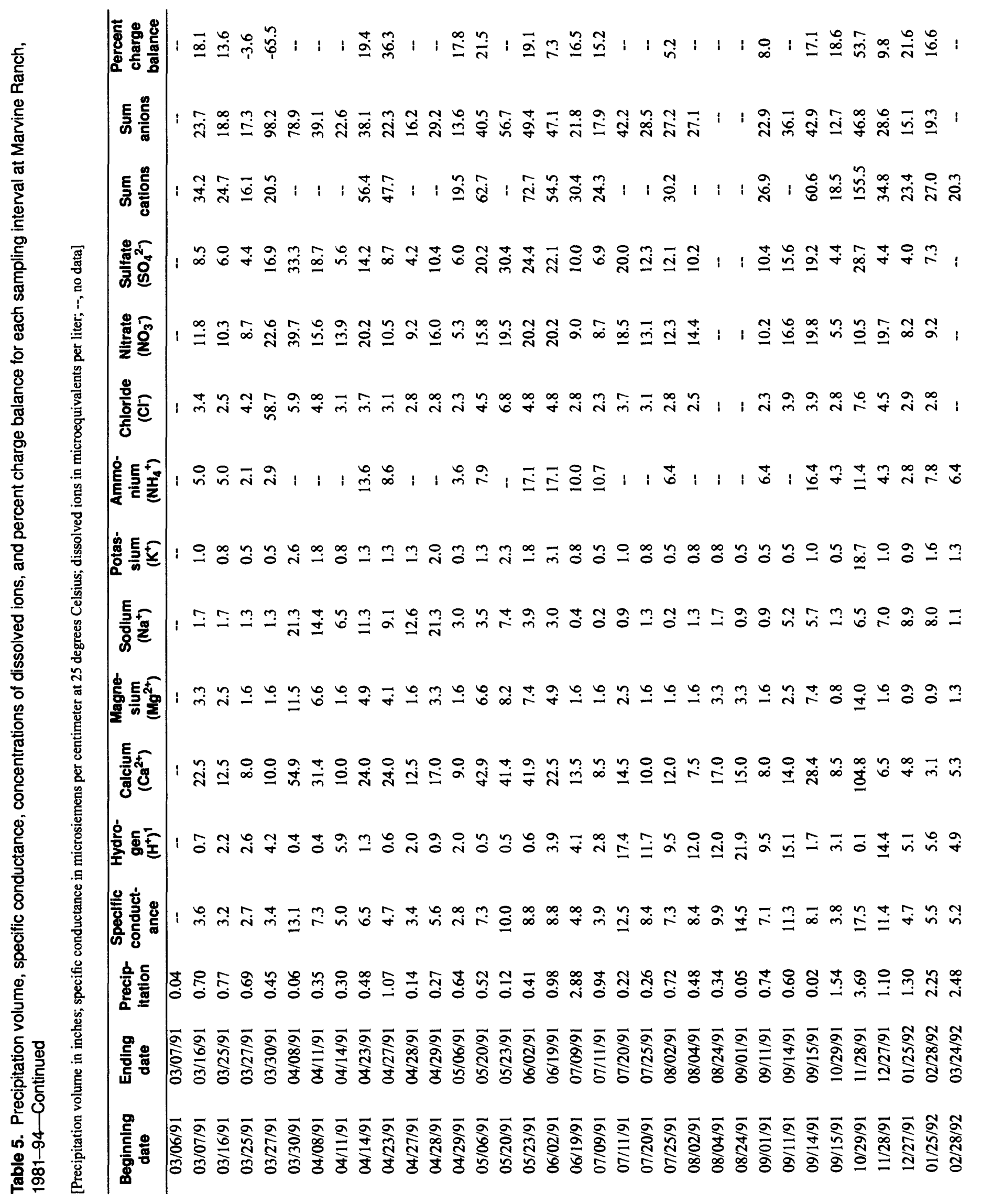




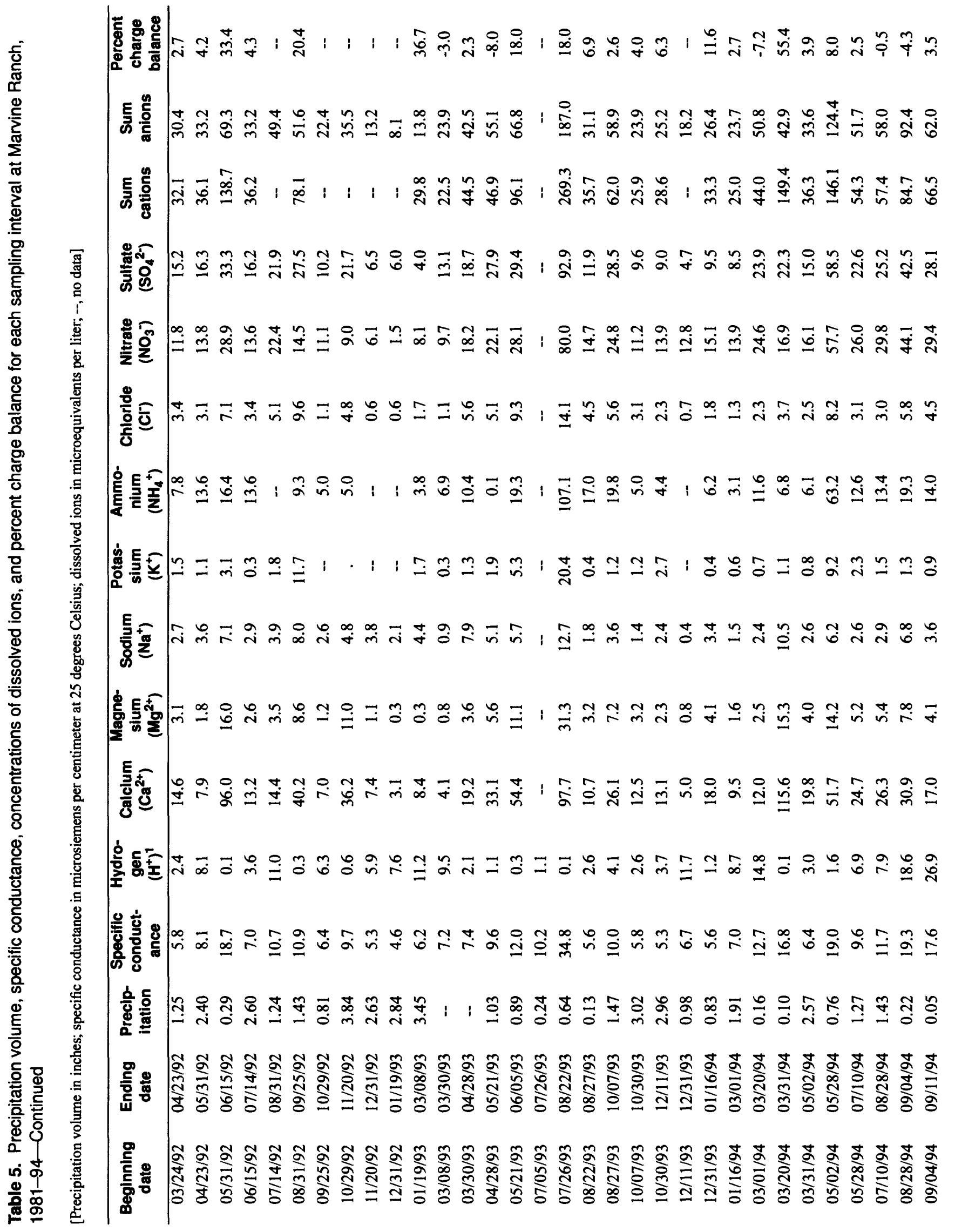




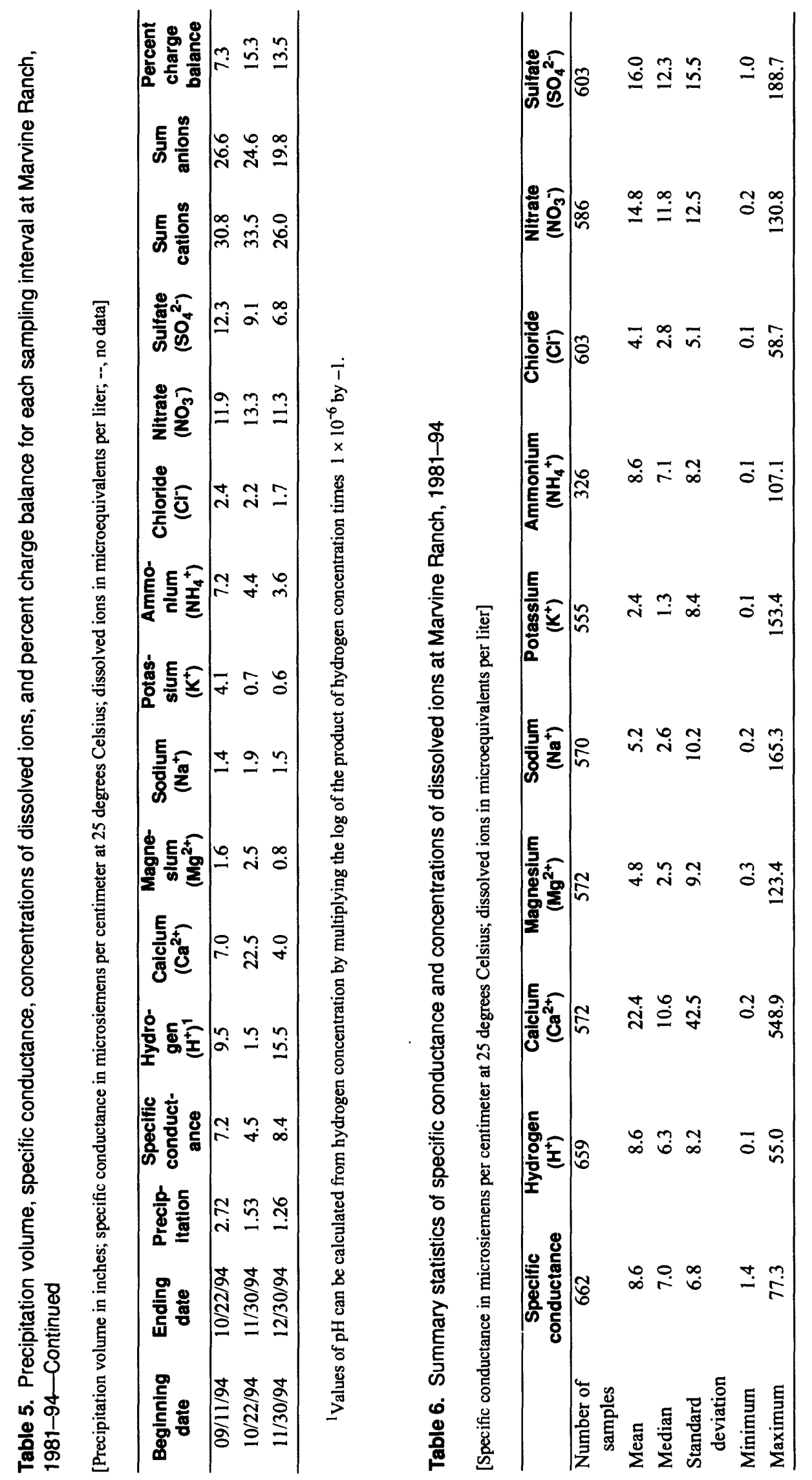




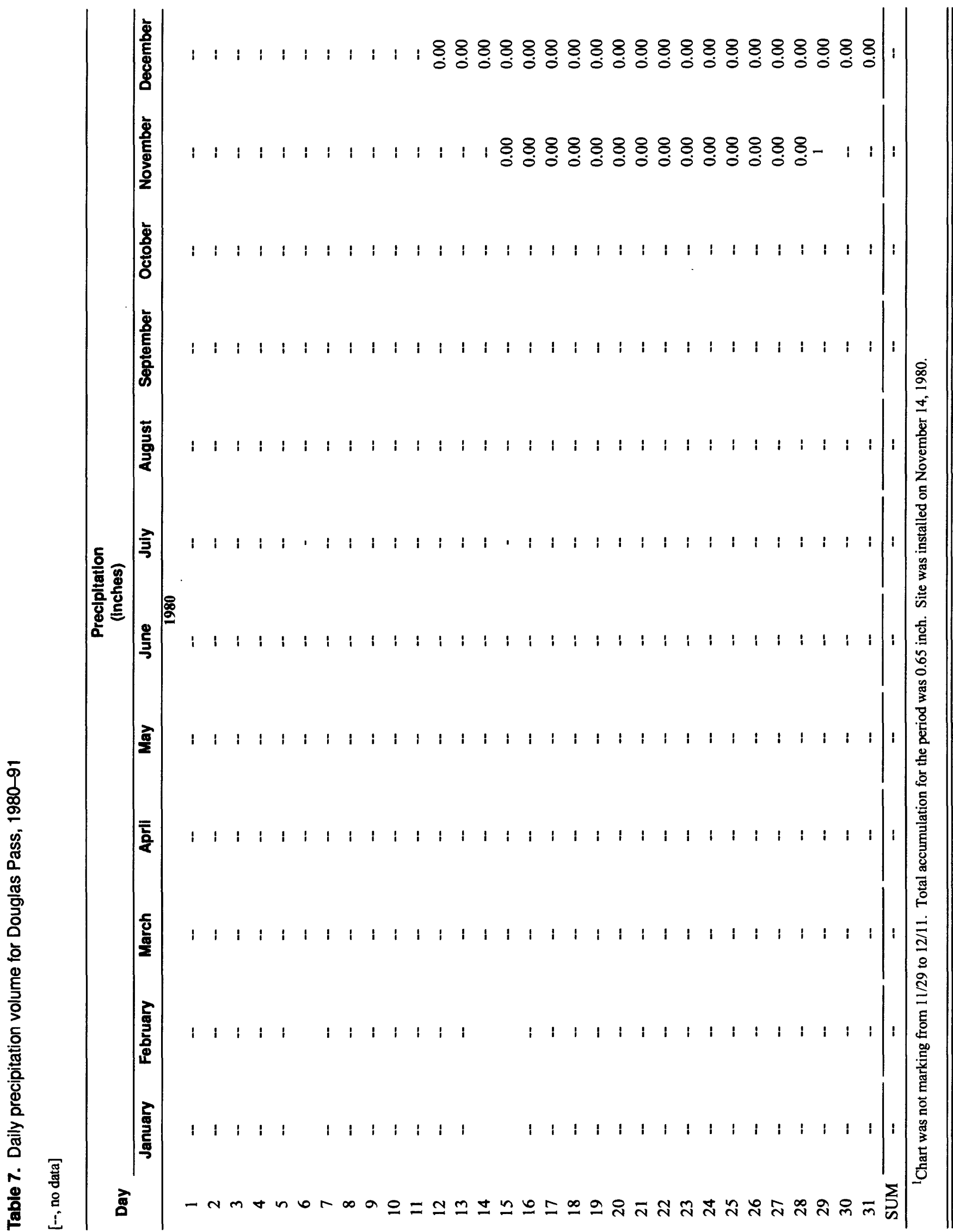




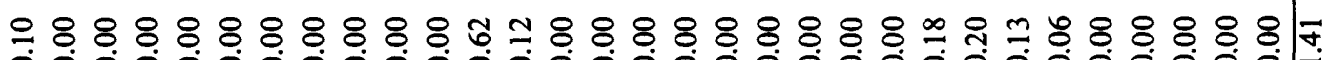
웅 융 웅 웅 웅 8 웅

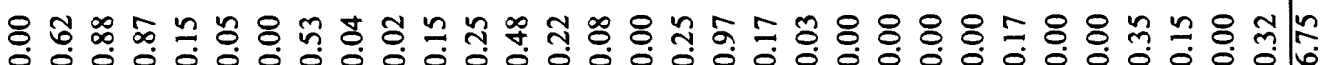

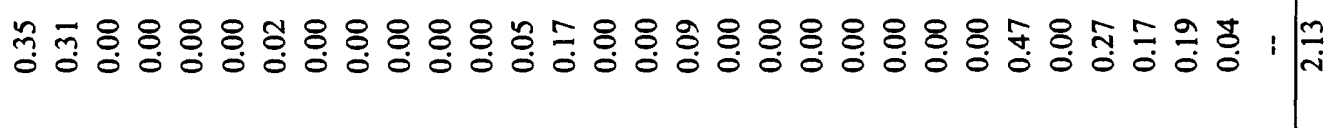

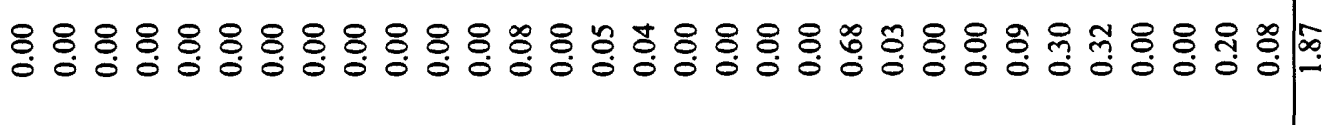

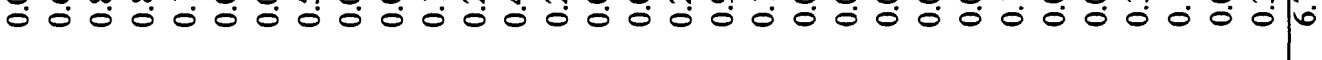

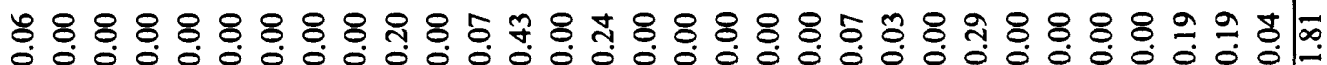

눙

ה 更

ำ ๆ o 0 O 00000

8는

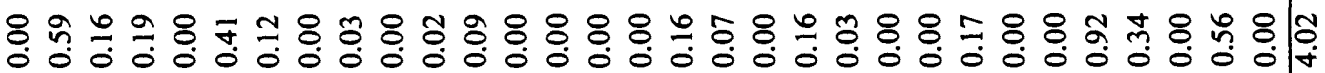

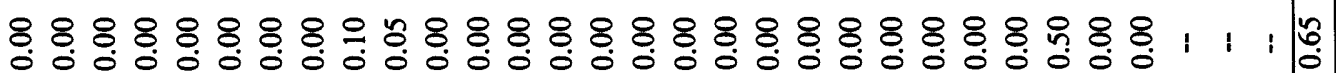

8. 8 


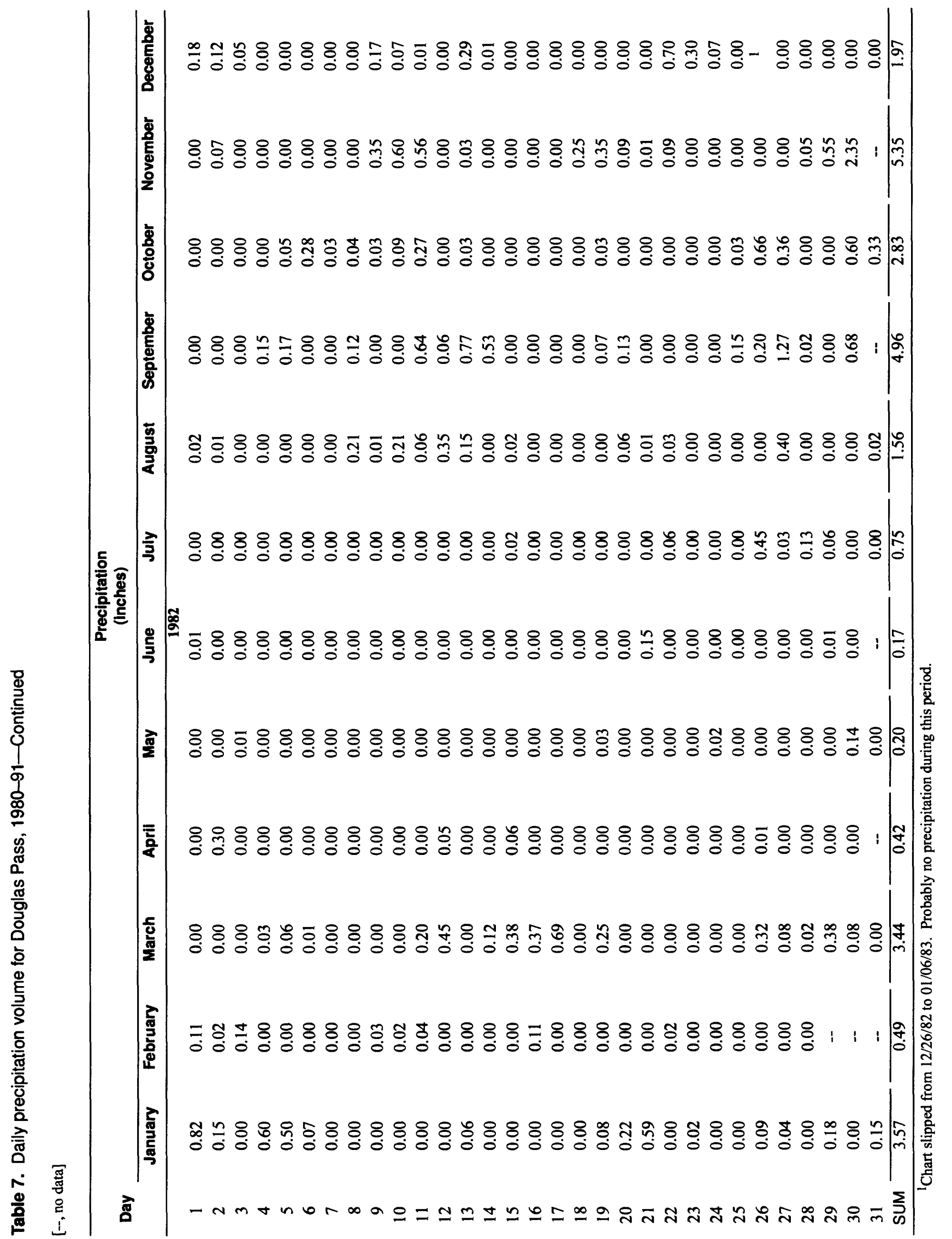




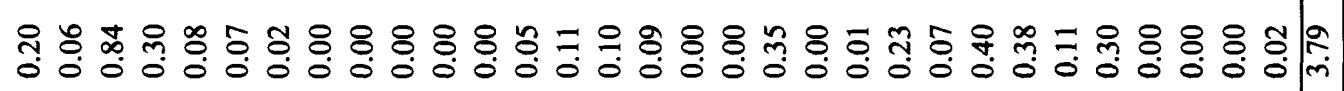

\&

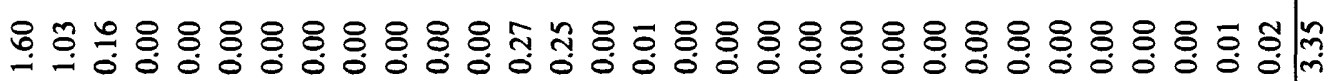

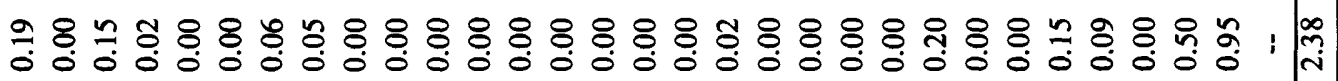

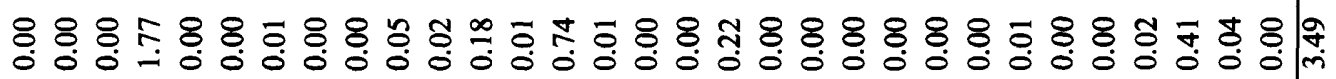

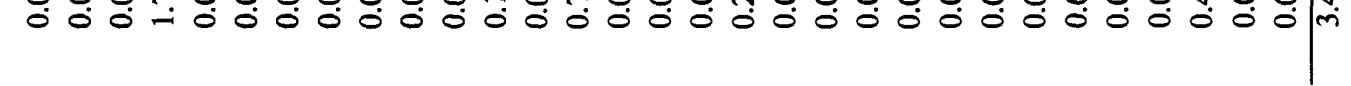

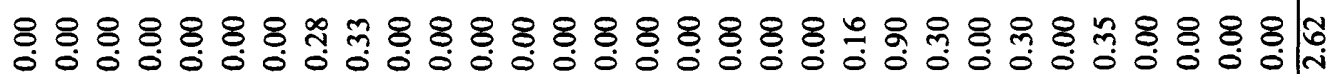

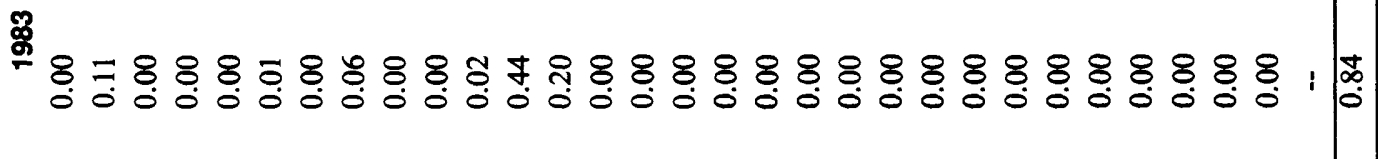

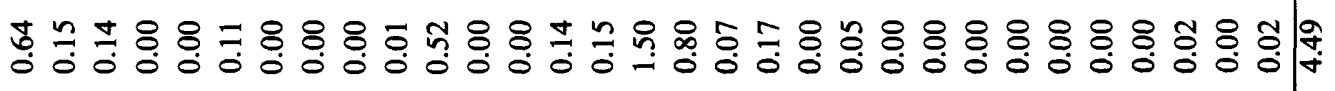

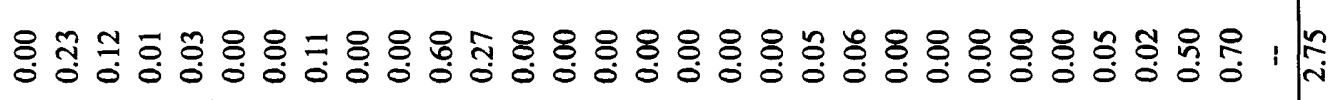

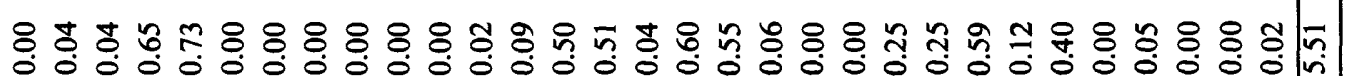
0.00000000000000000000000000000 nn

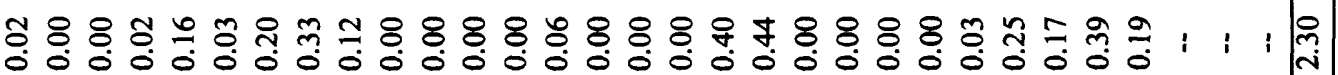
0.000 .0000000000000000000000114

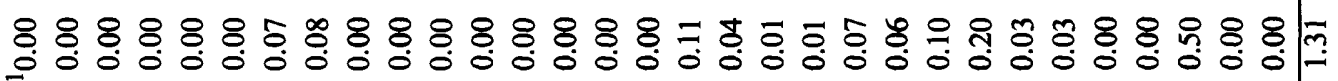




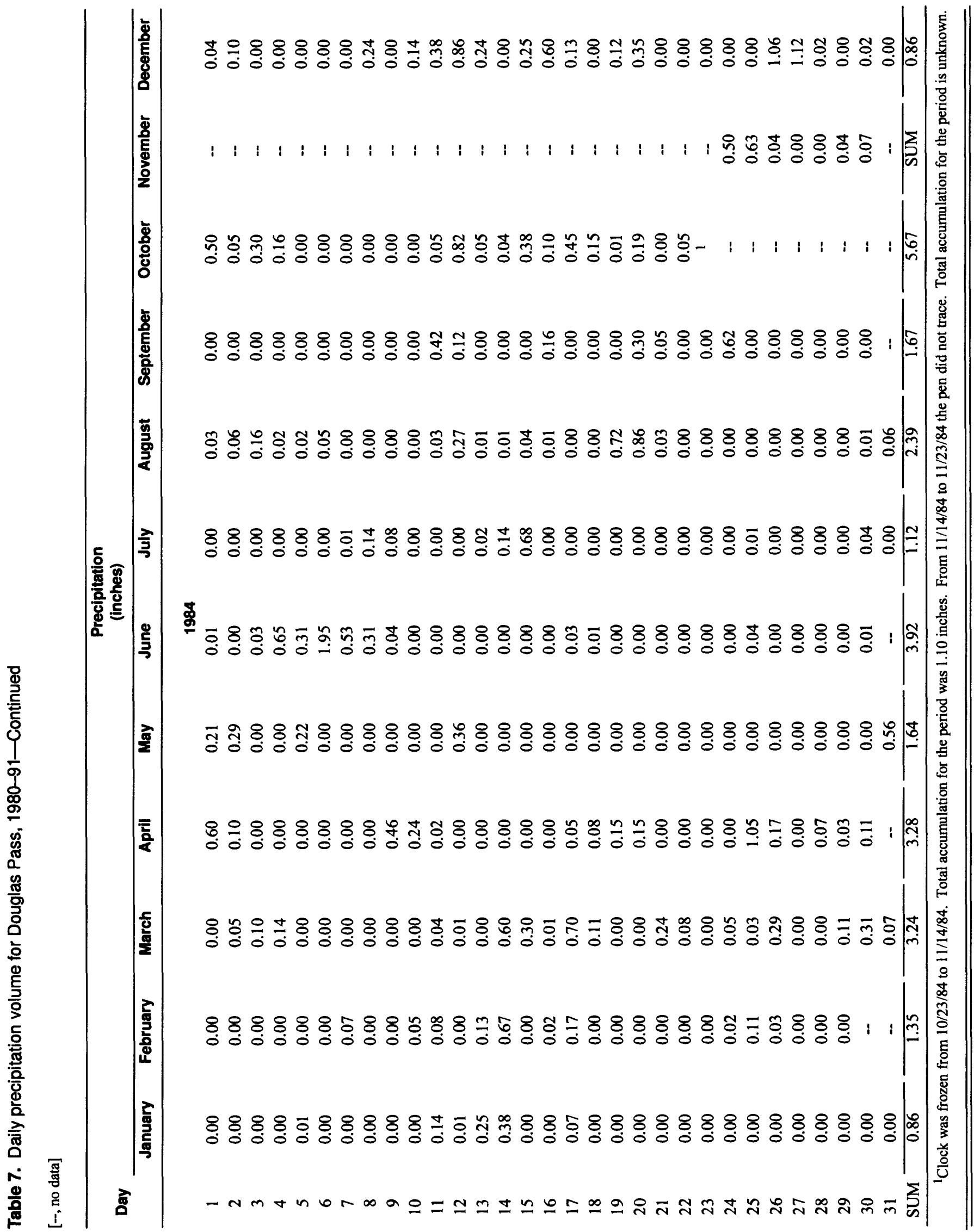




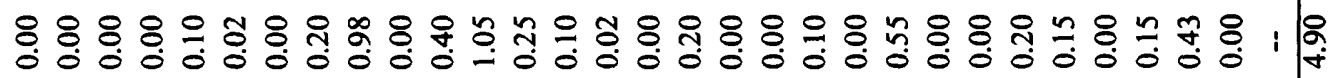

\&:융 \& \& \& \&

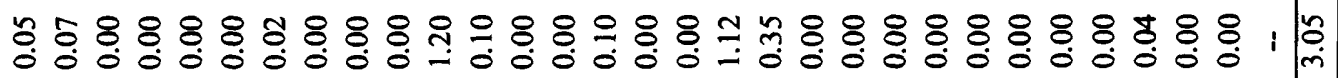

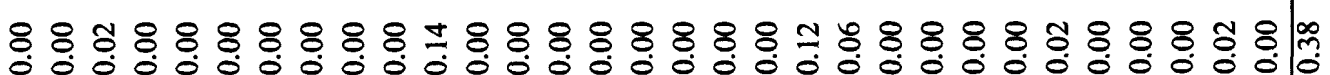

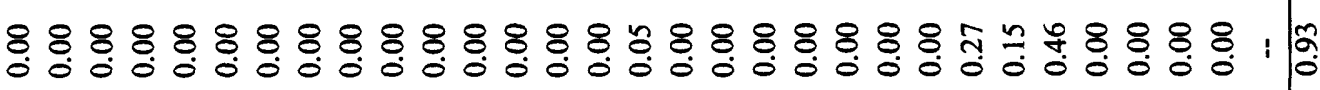

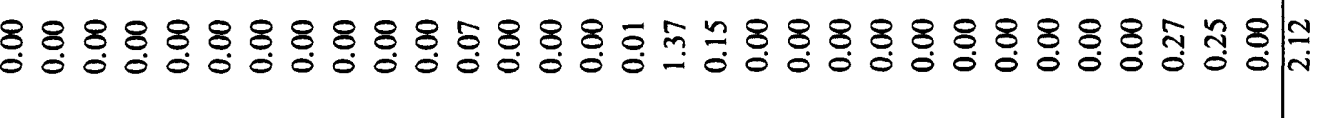

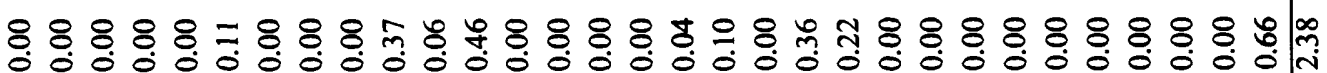

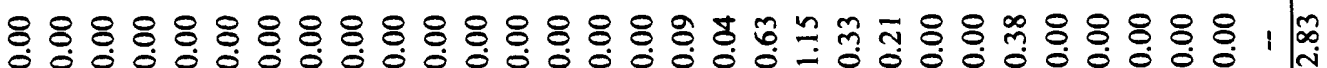

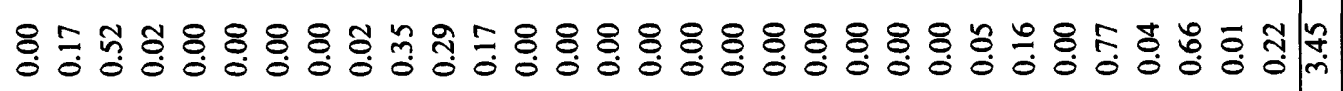

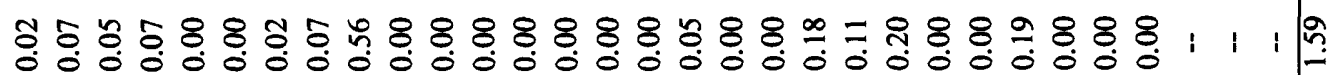

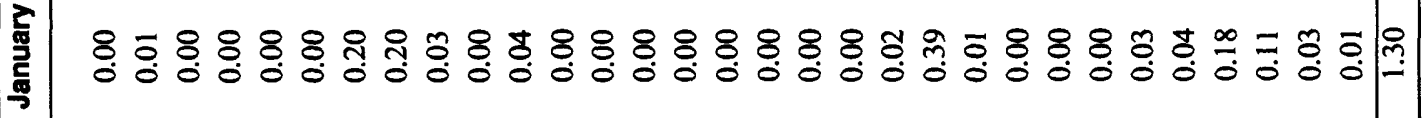




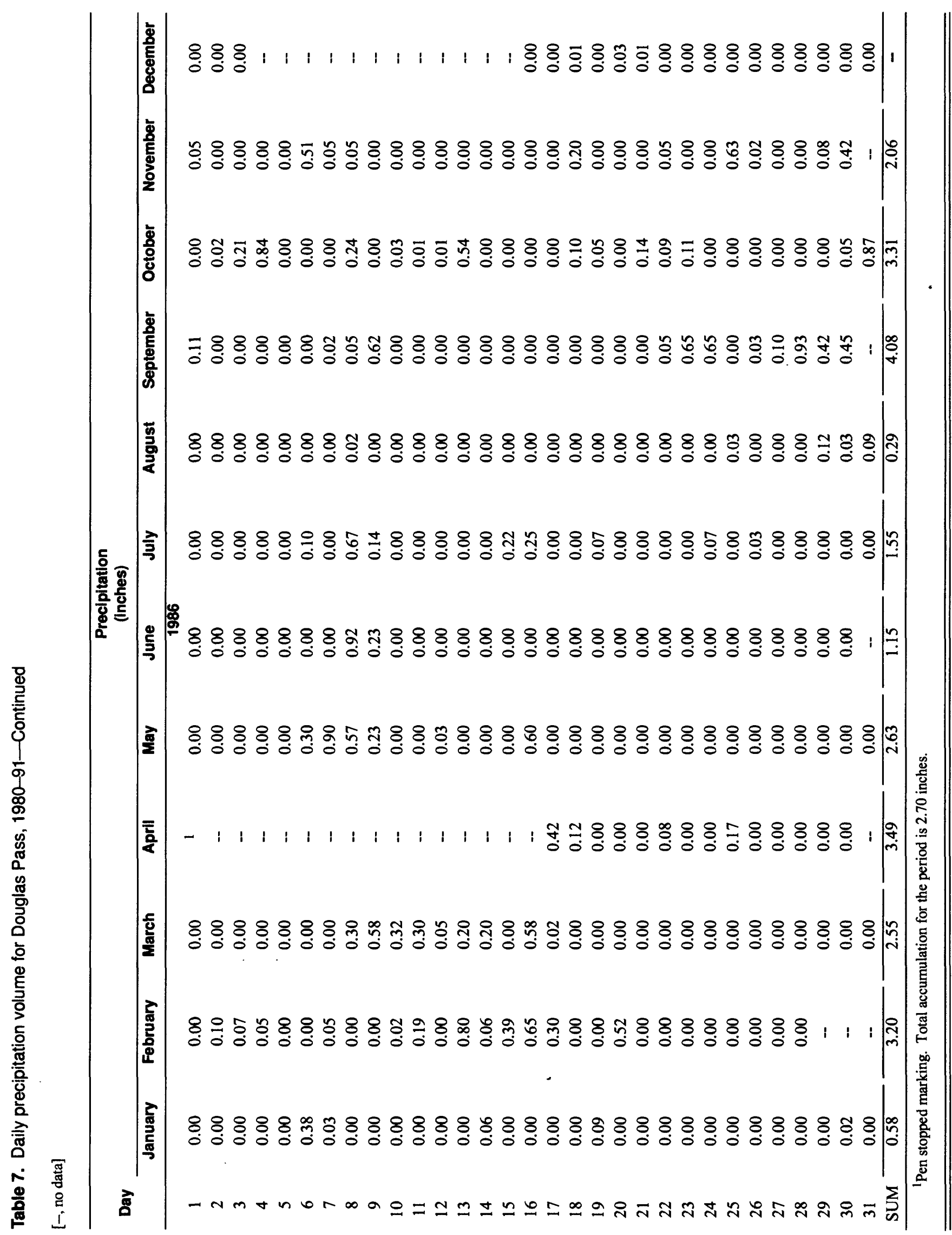




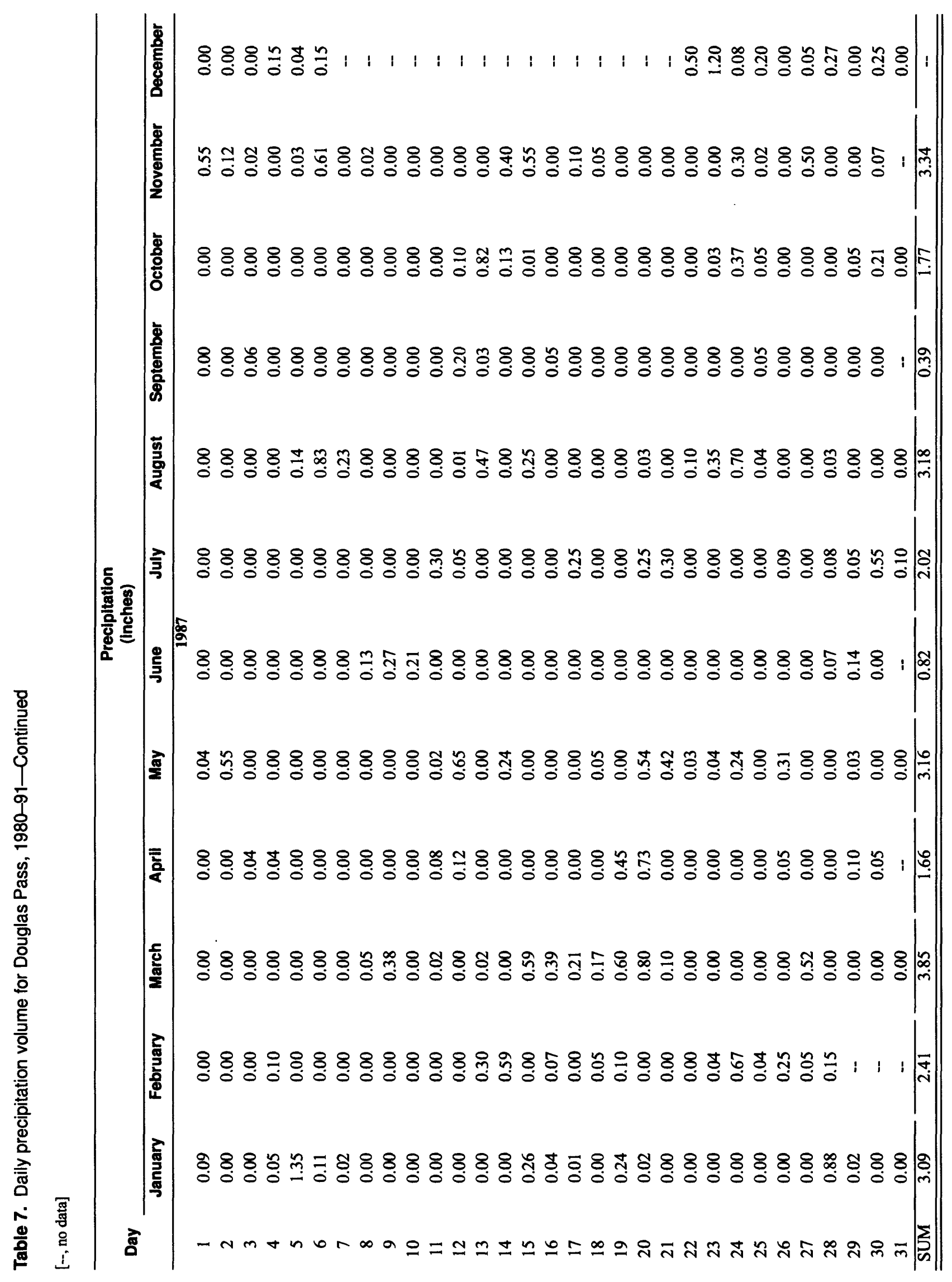




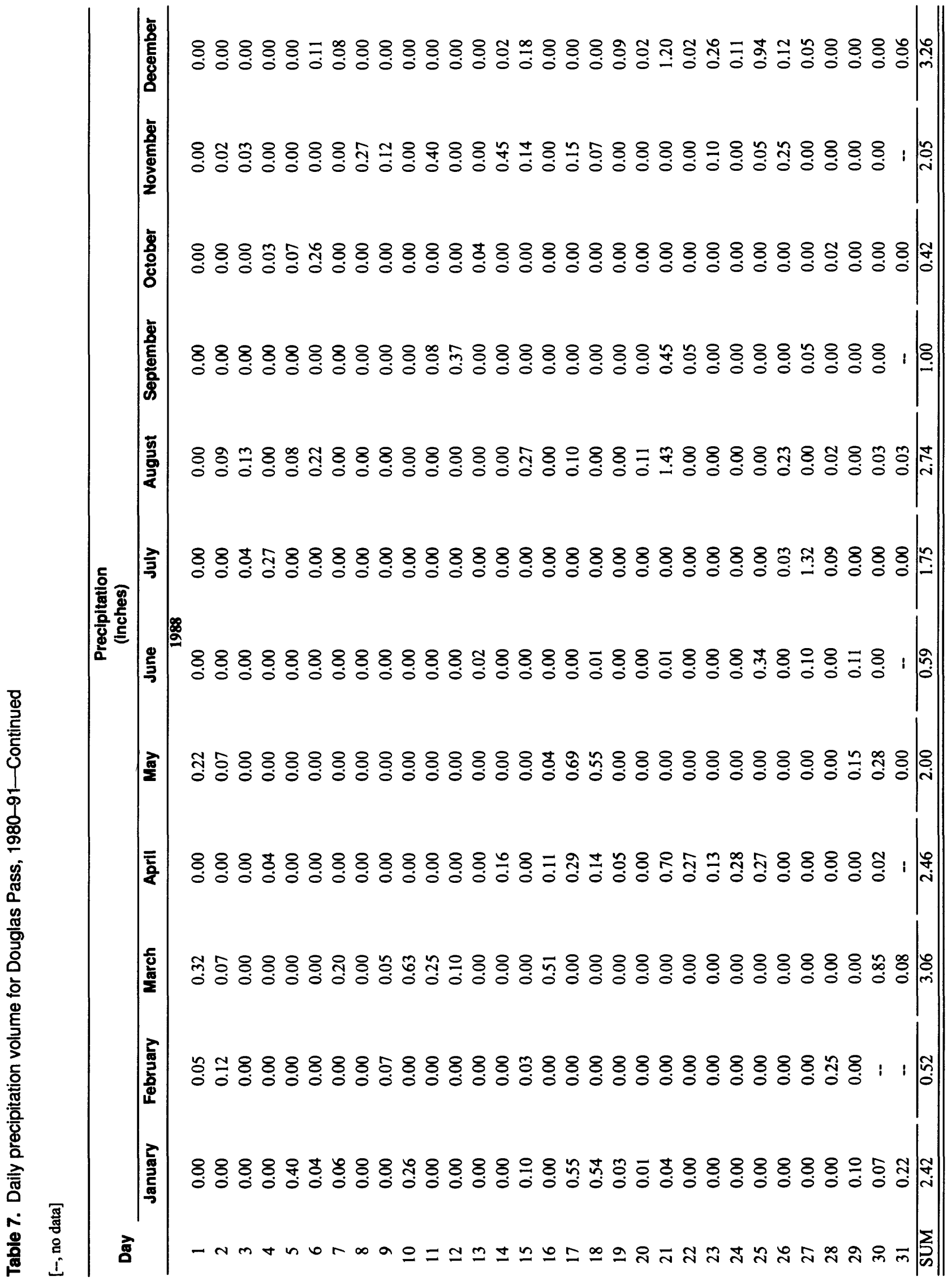




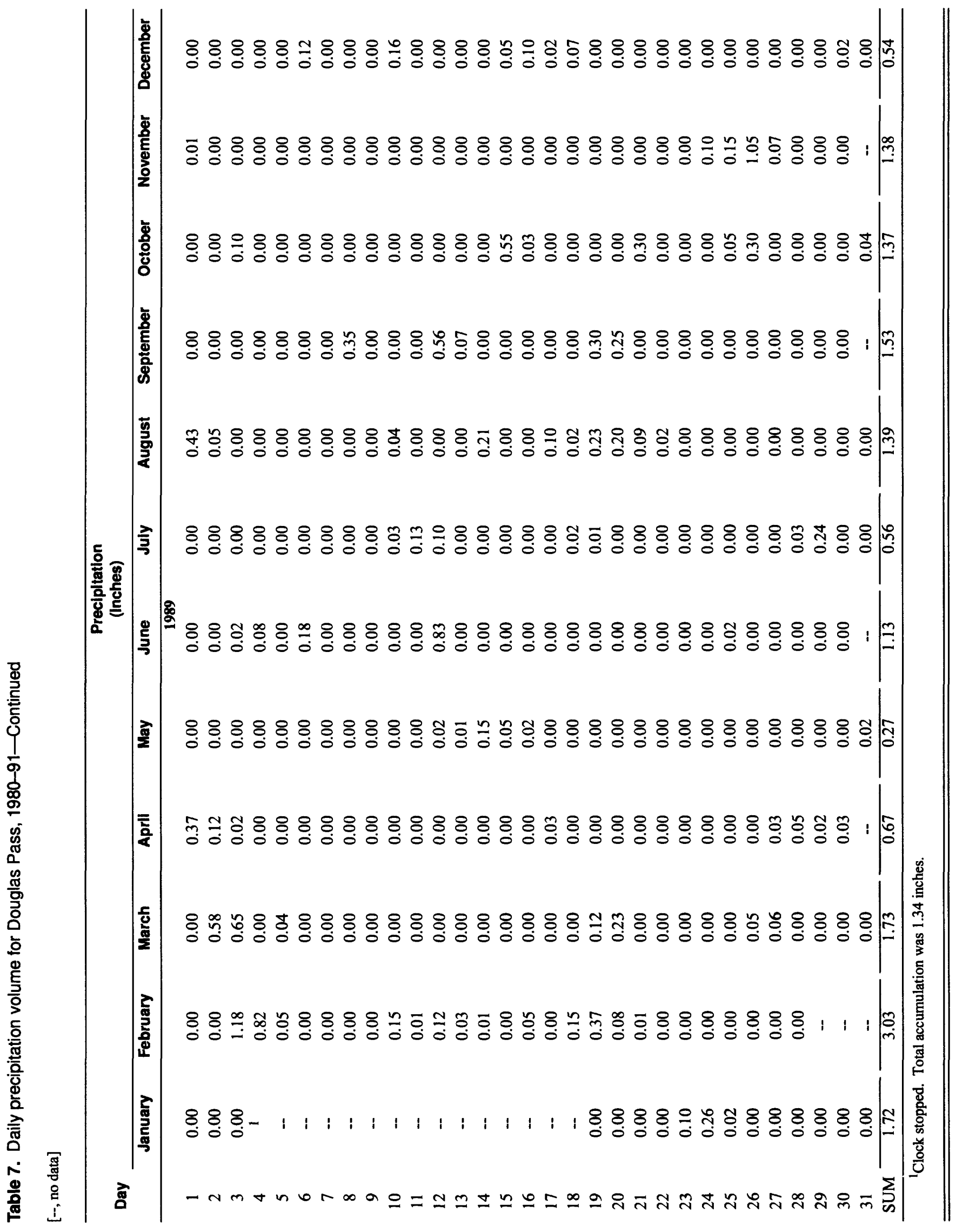




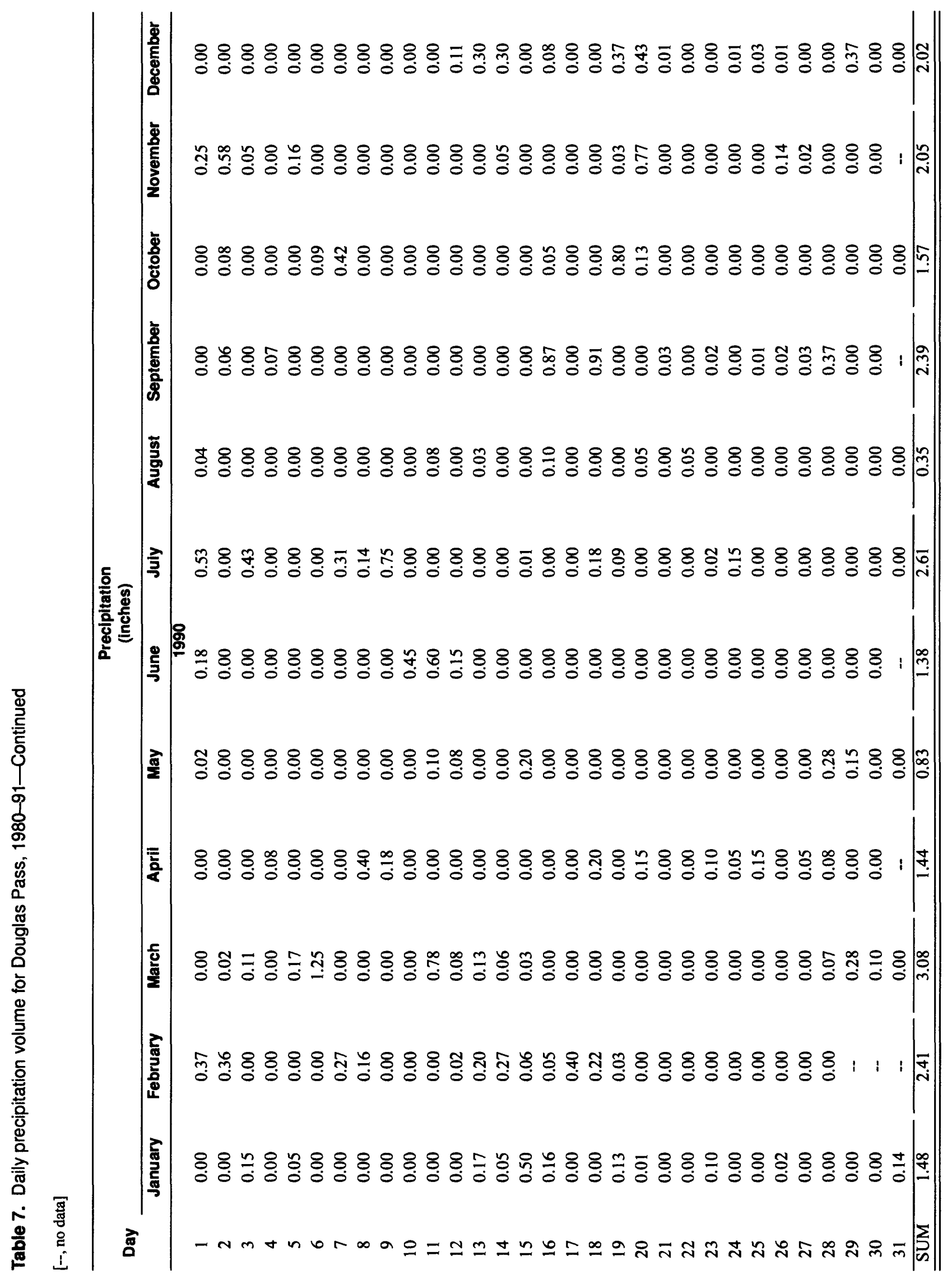




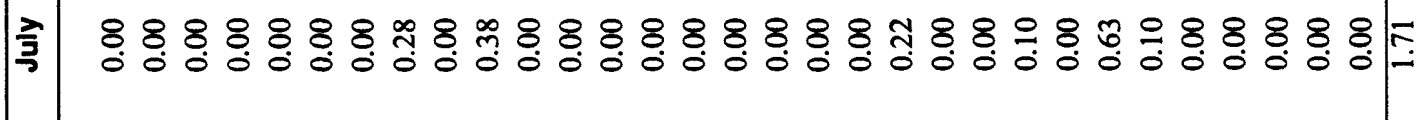

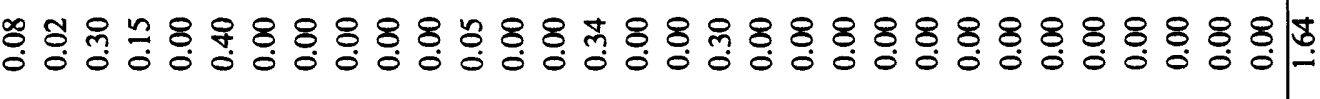

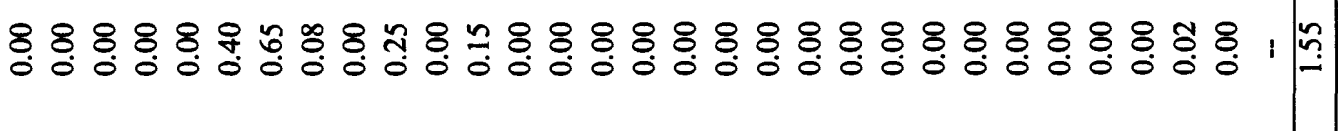

$\begin{array}{lllllllllllllllllllllllllllllllll} & 1 & 1 & 1 & 1 & 1 & 1 & 1 & 1 & 1 & 1 & 1 & 1 & 1 & 1 & 1 & 1 & 1 & 1 & 1 & 1 & 1 & 1 & 1 & 1 & 1 & 1 & 1 & 1 & 1 & 1 & 1\end{array}$

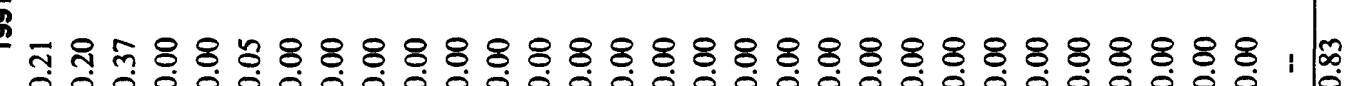
잉

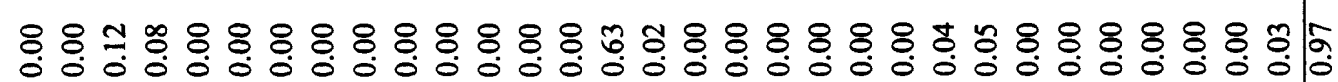

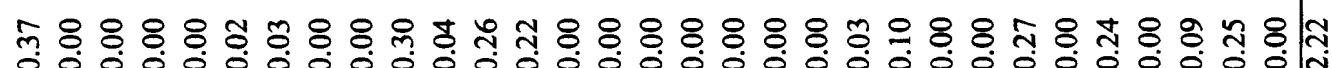

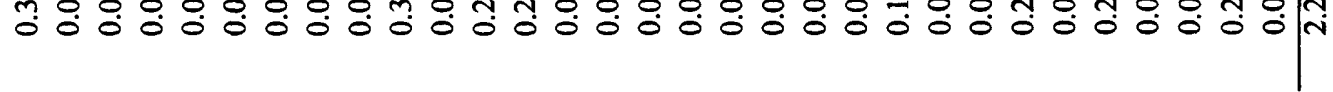

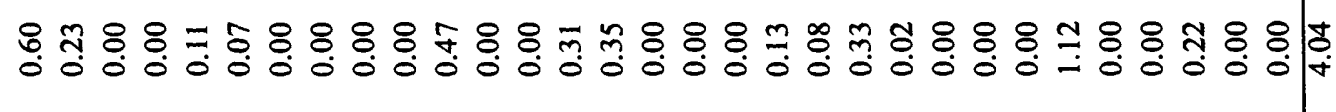

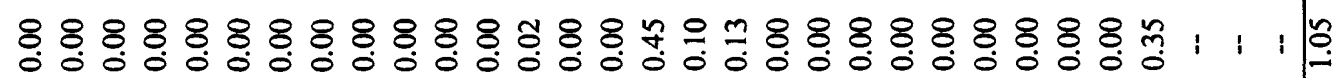

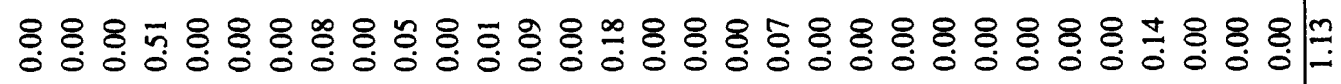




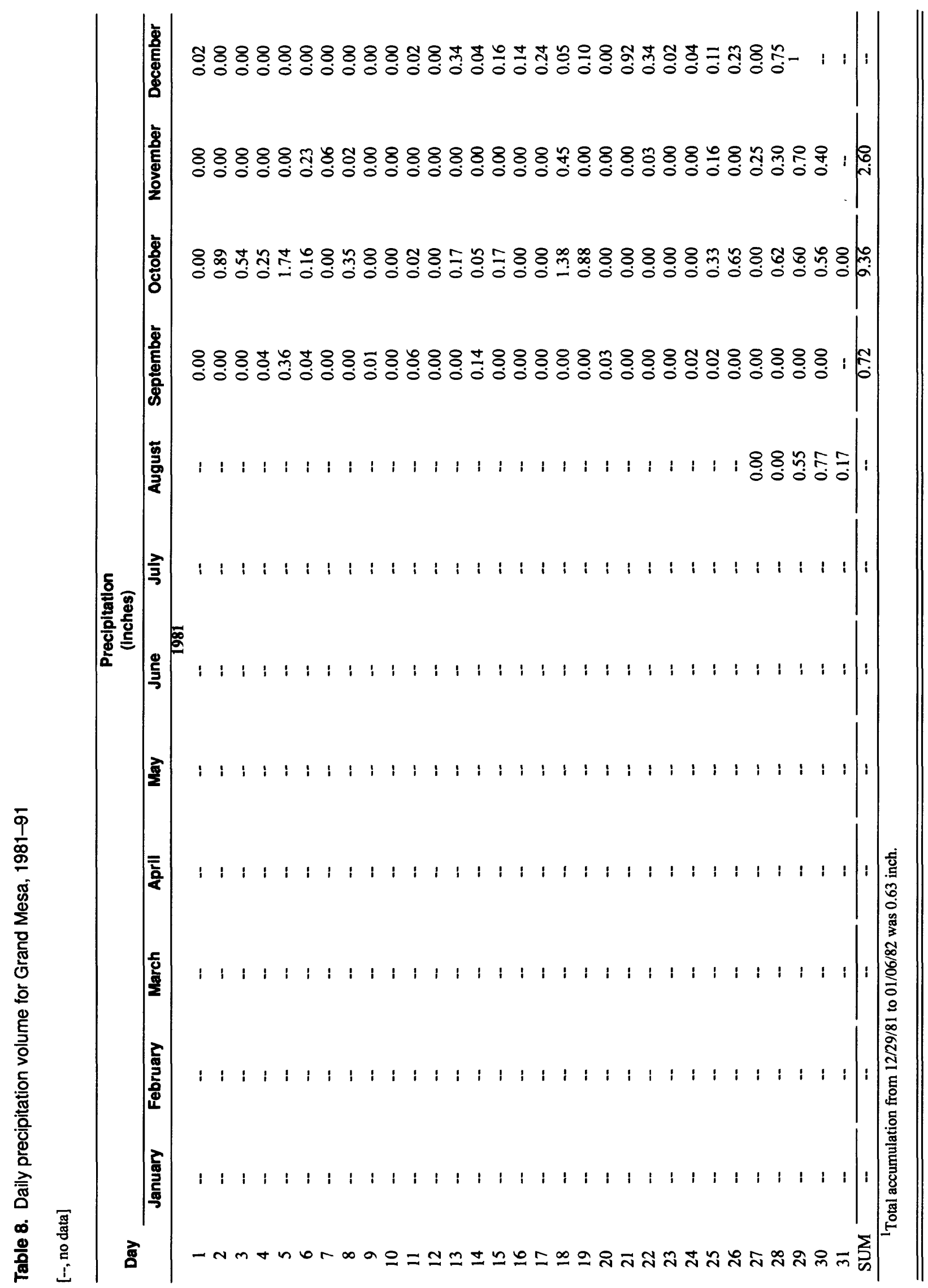




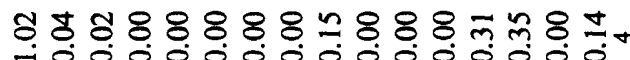

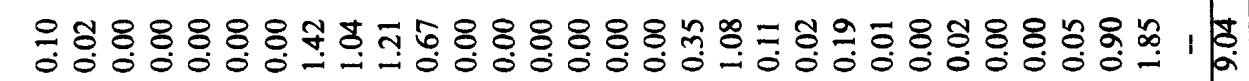

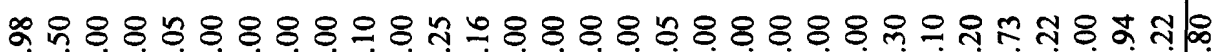

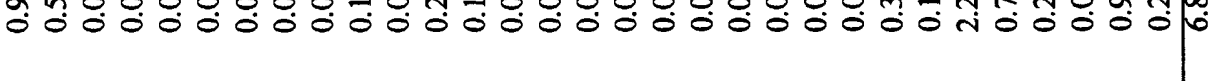

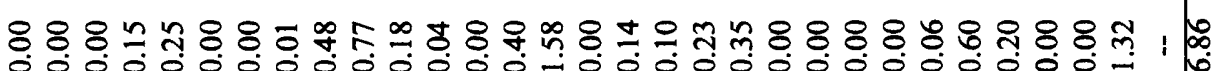

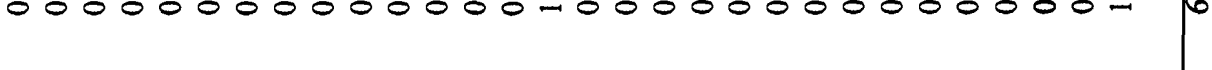

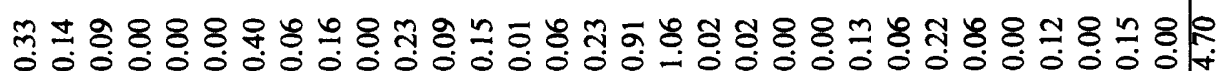

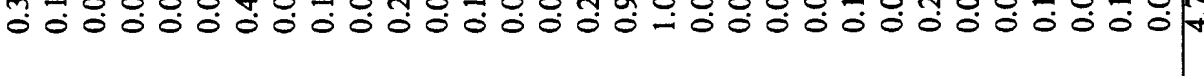

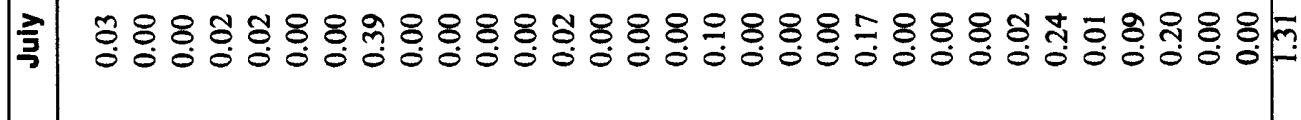
$\approx$

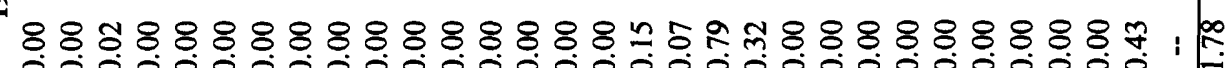
(2)

๑๐士

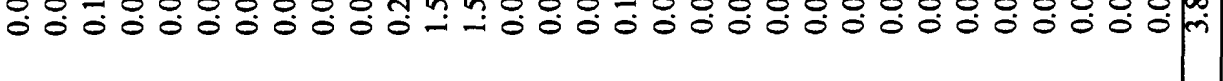

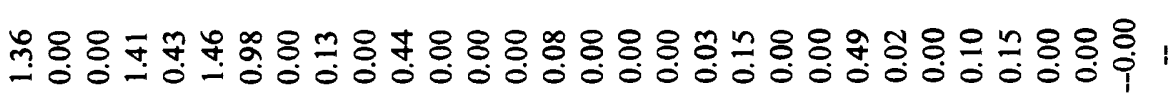

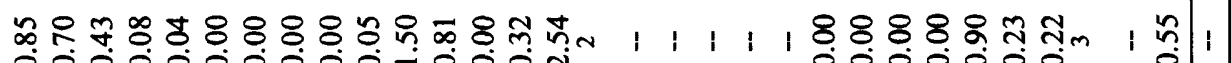

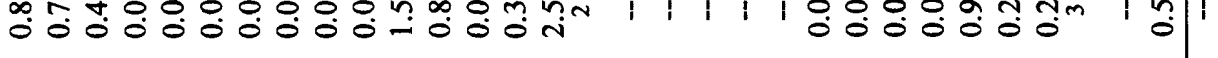
然

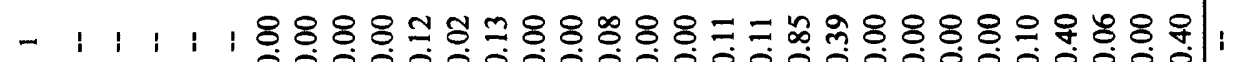




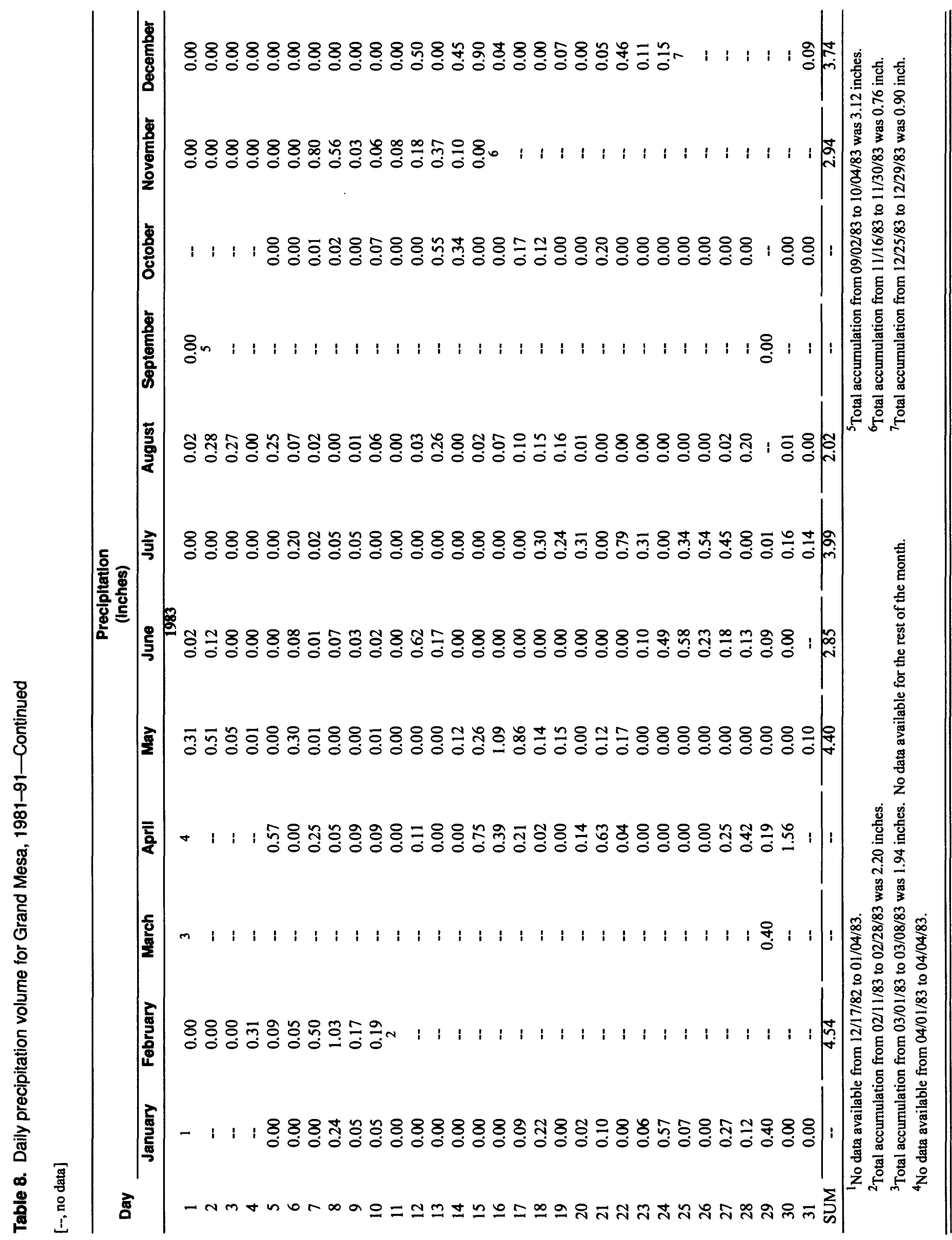




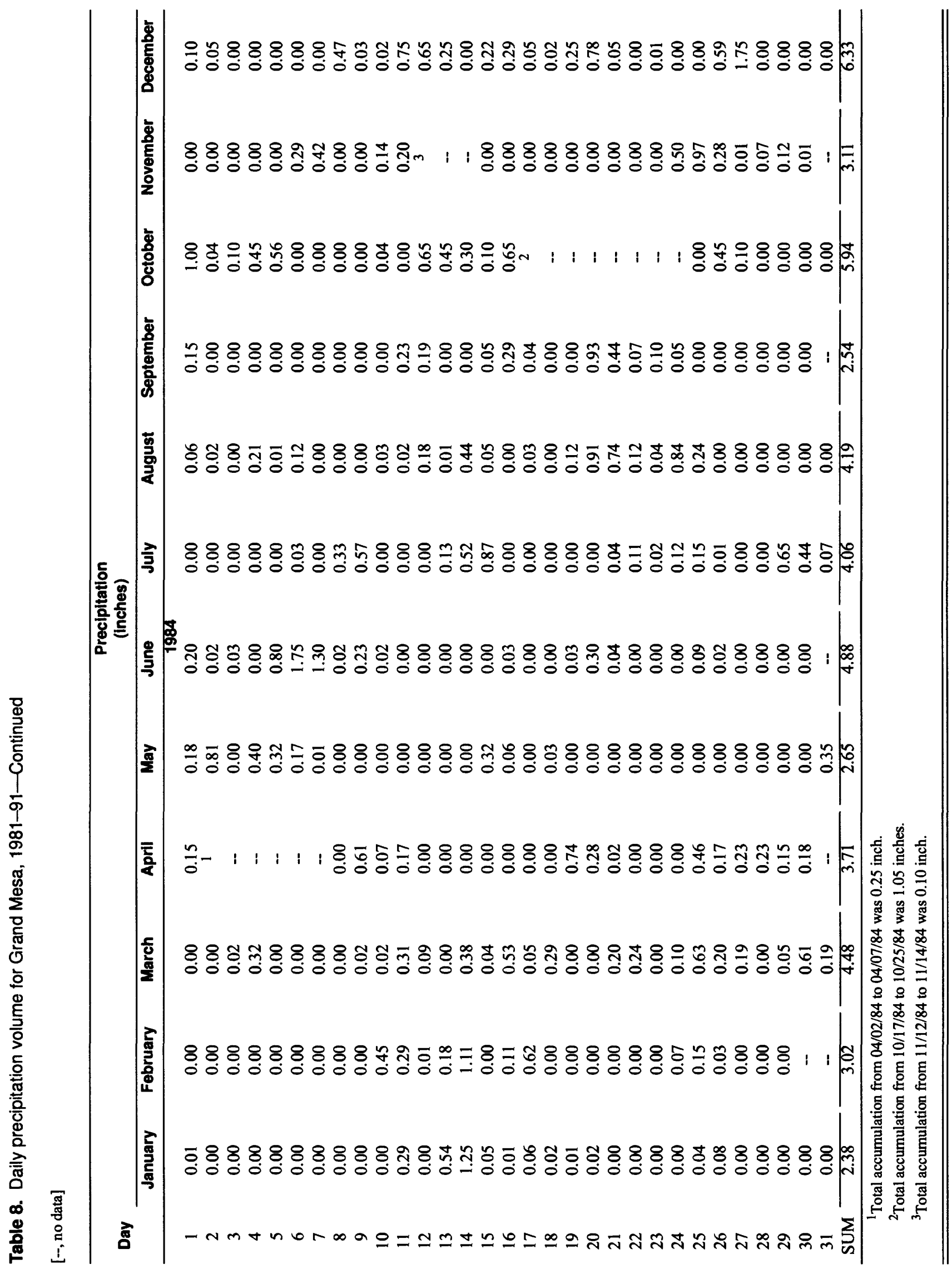




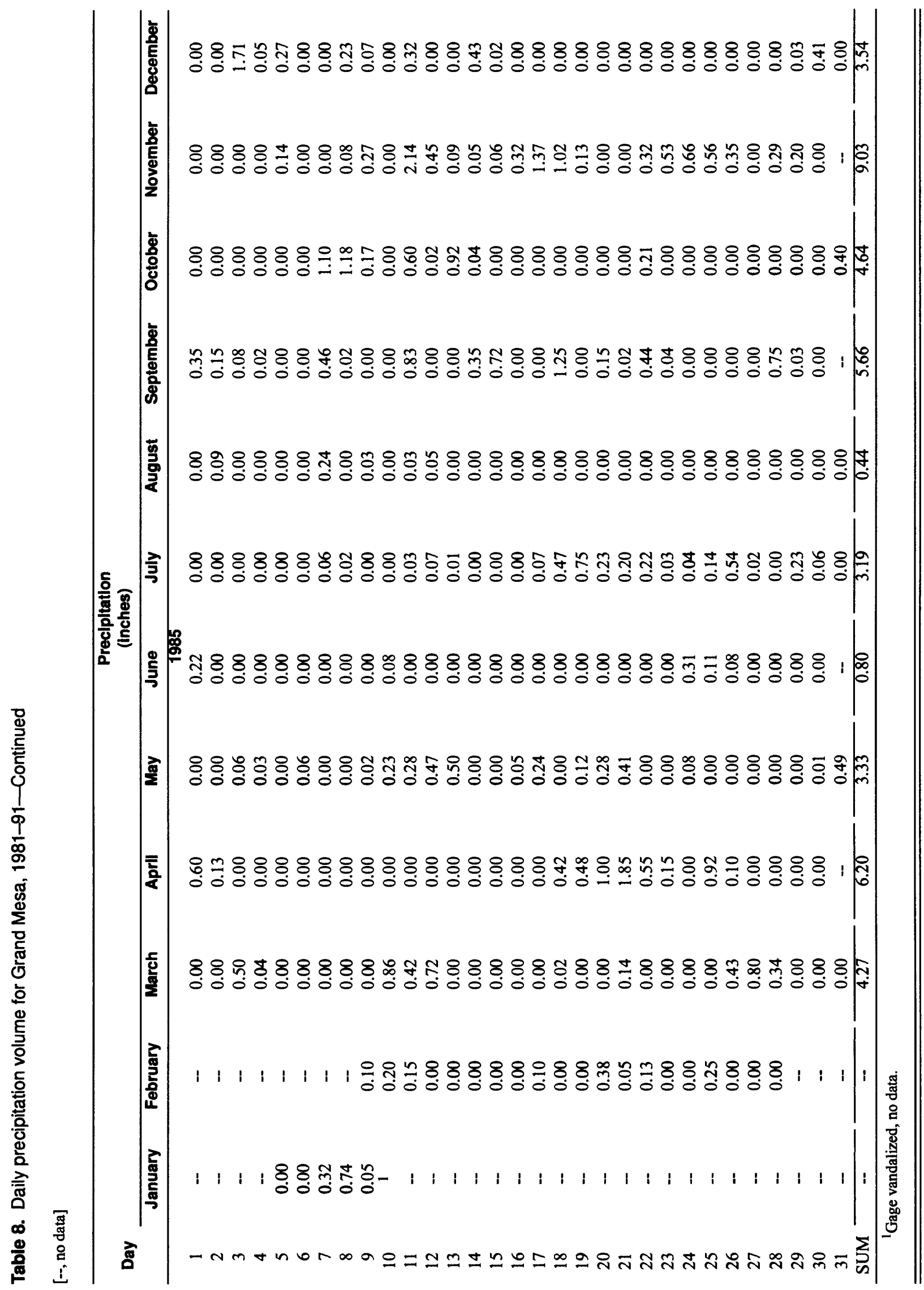




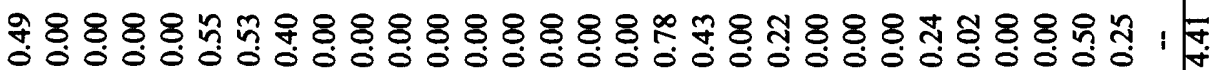

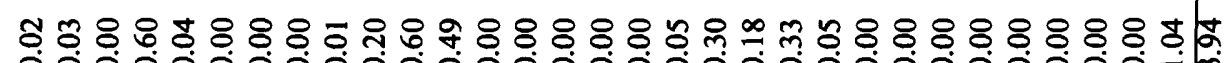
0000000000000000000000000000000 m

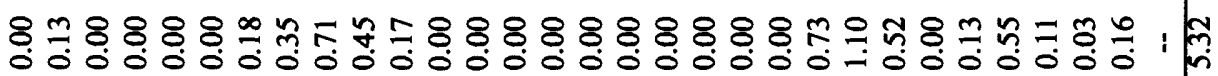

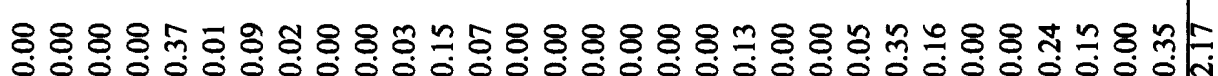

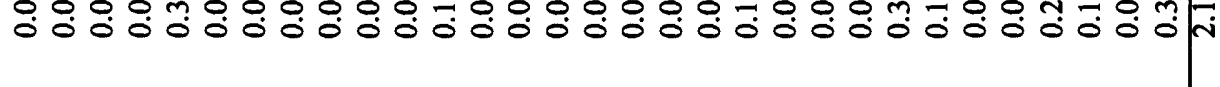

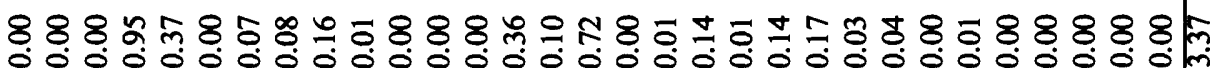

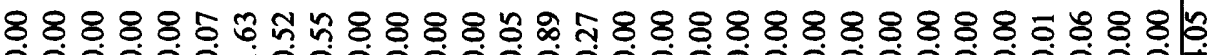

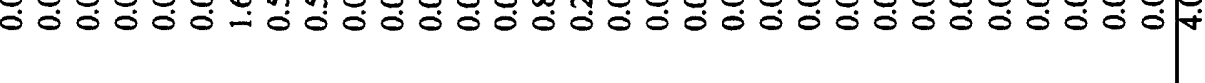

8.융유. 


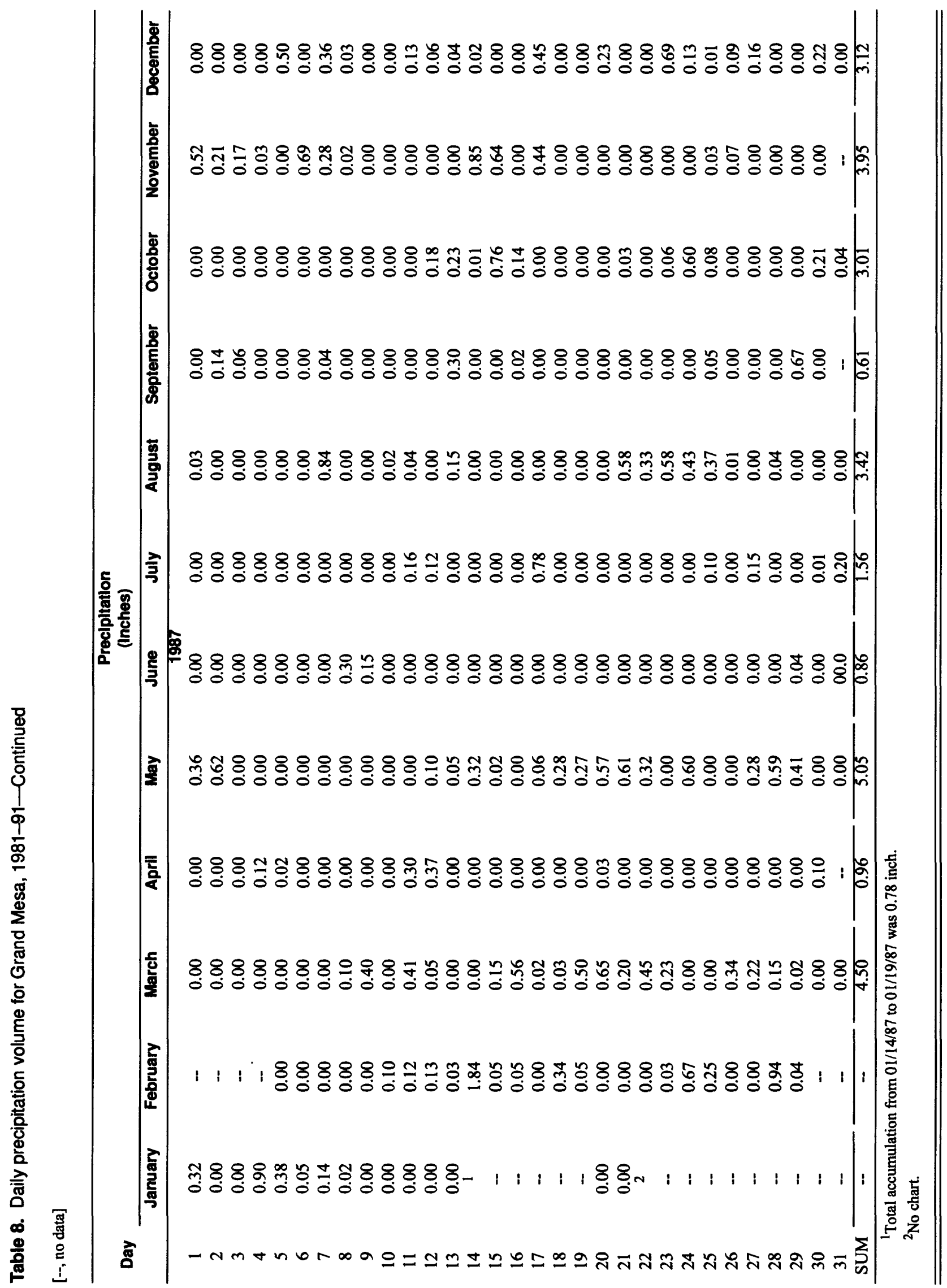




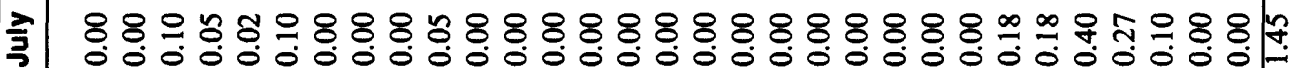

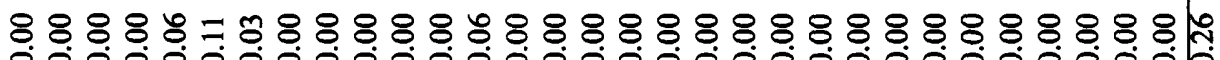
00000000000000000000000000000000 88808888080

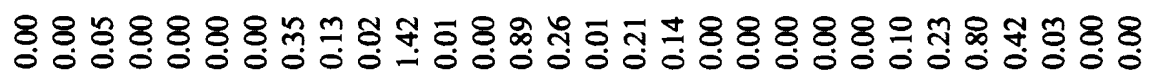

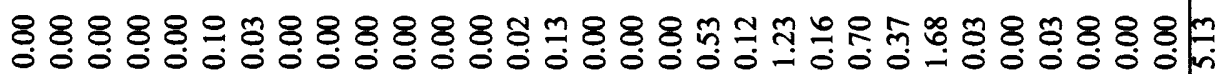

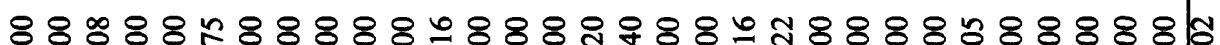

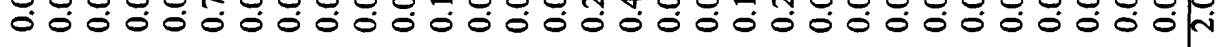

$4: 1488888 \% 8888888888888 \% 850$ \%

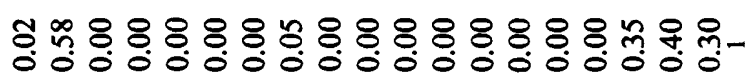

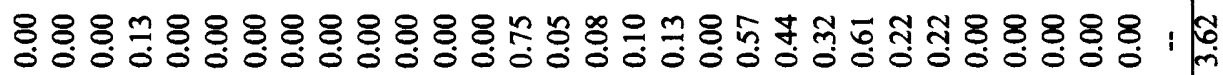

ஸ

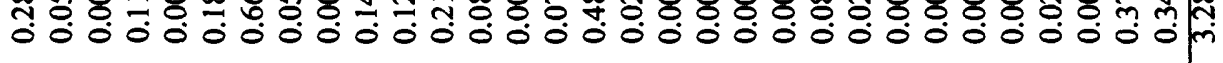

ธำร88888ษ

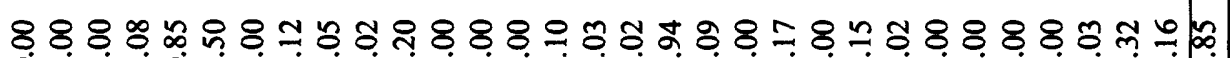

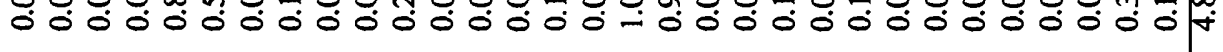




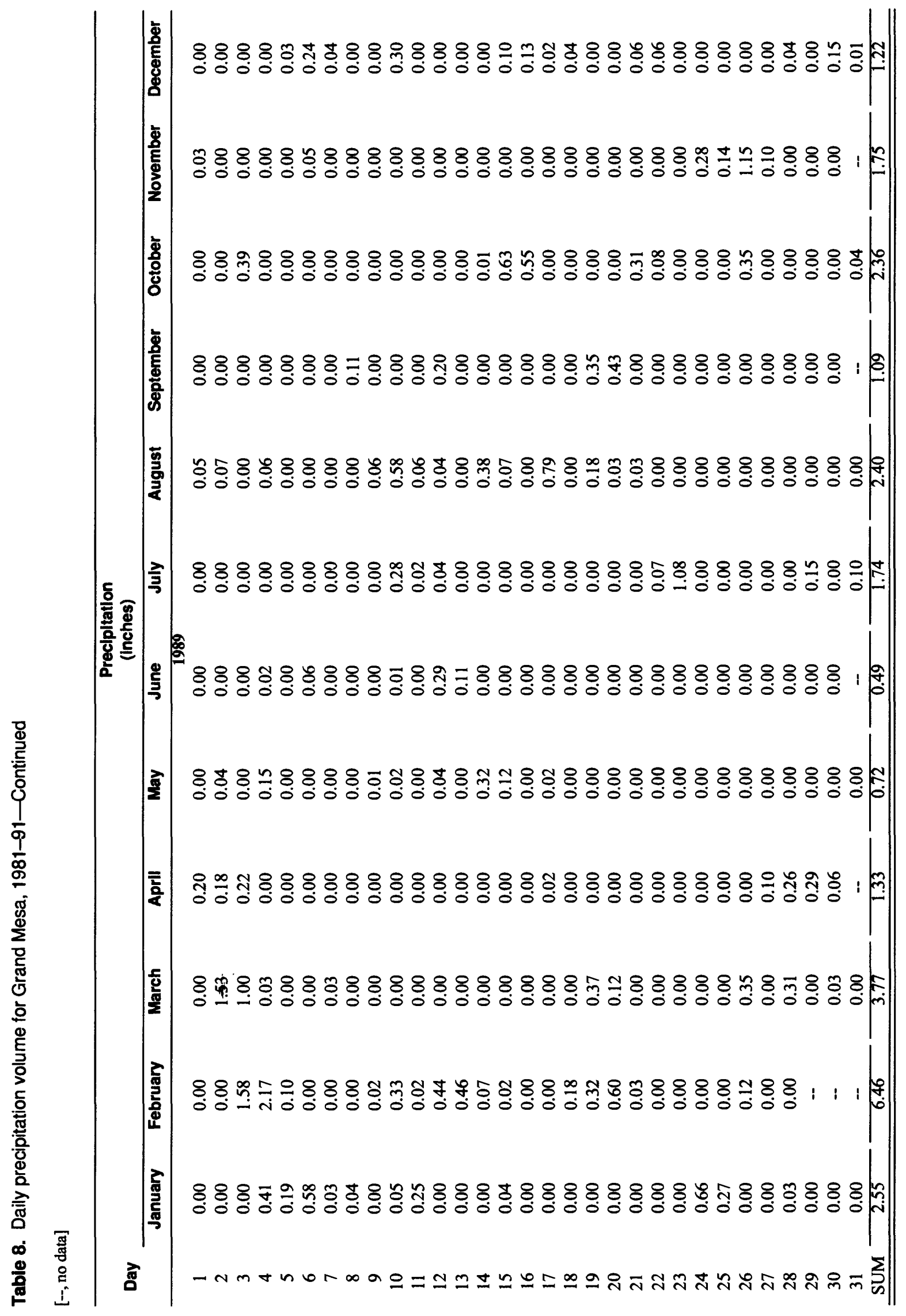




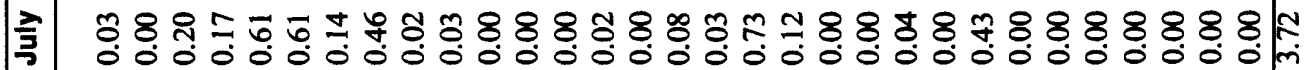

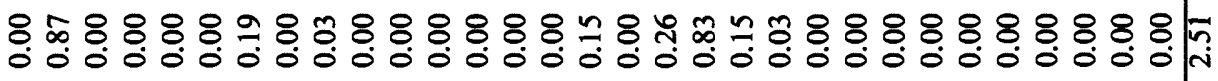

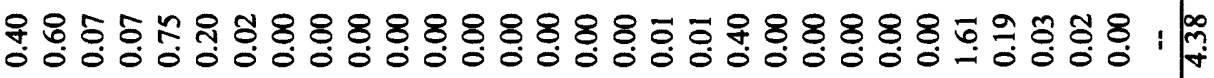

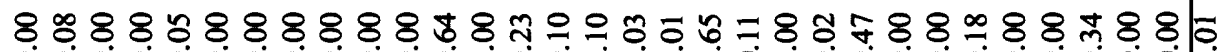
$000000000000-000000000000000000 \mid$

$8888 \% 8888888888 \%$ \%

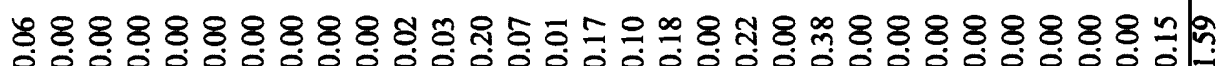

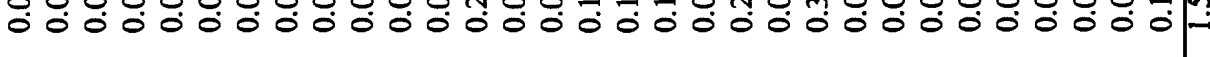

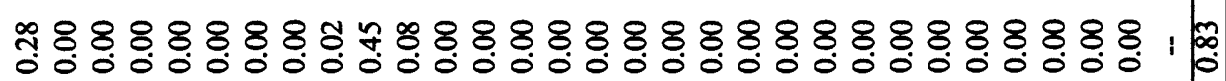

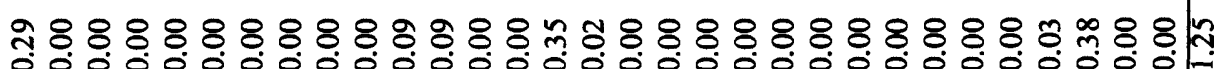

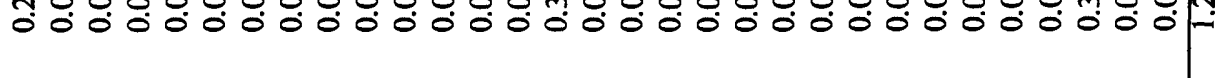

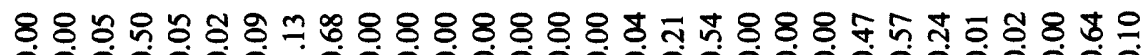

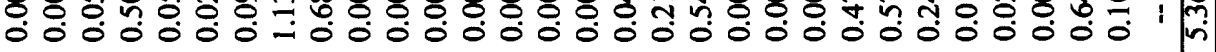

8. ำㄲํำ

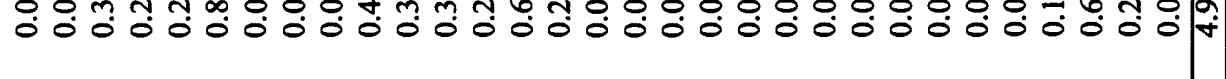
ڤ ○े

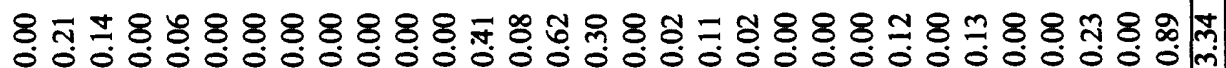
000000 


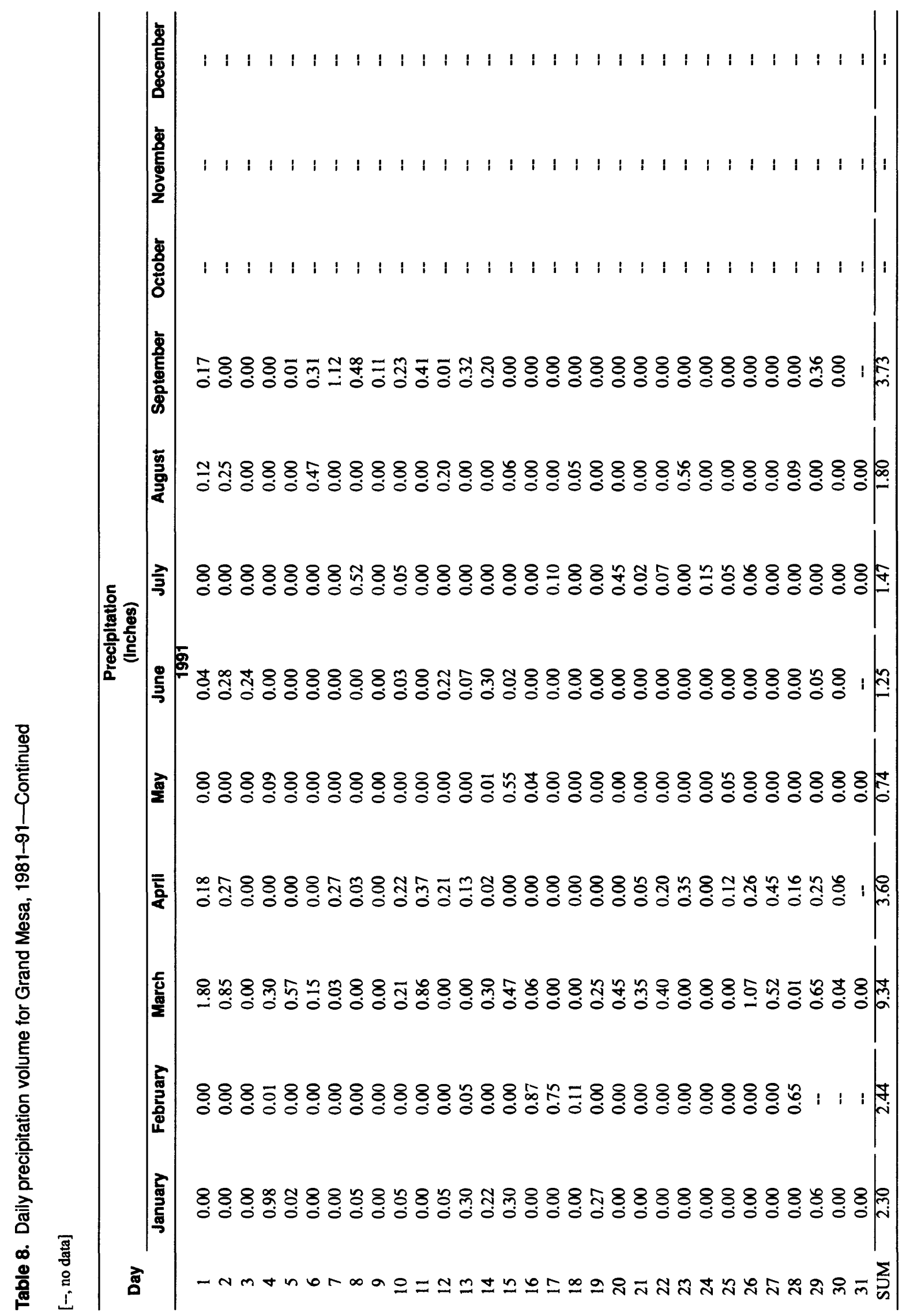




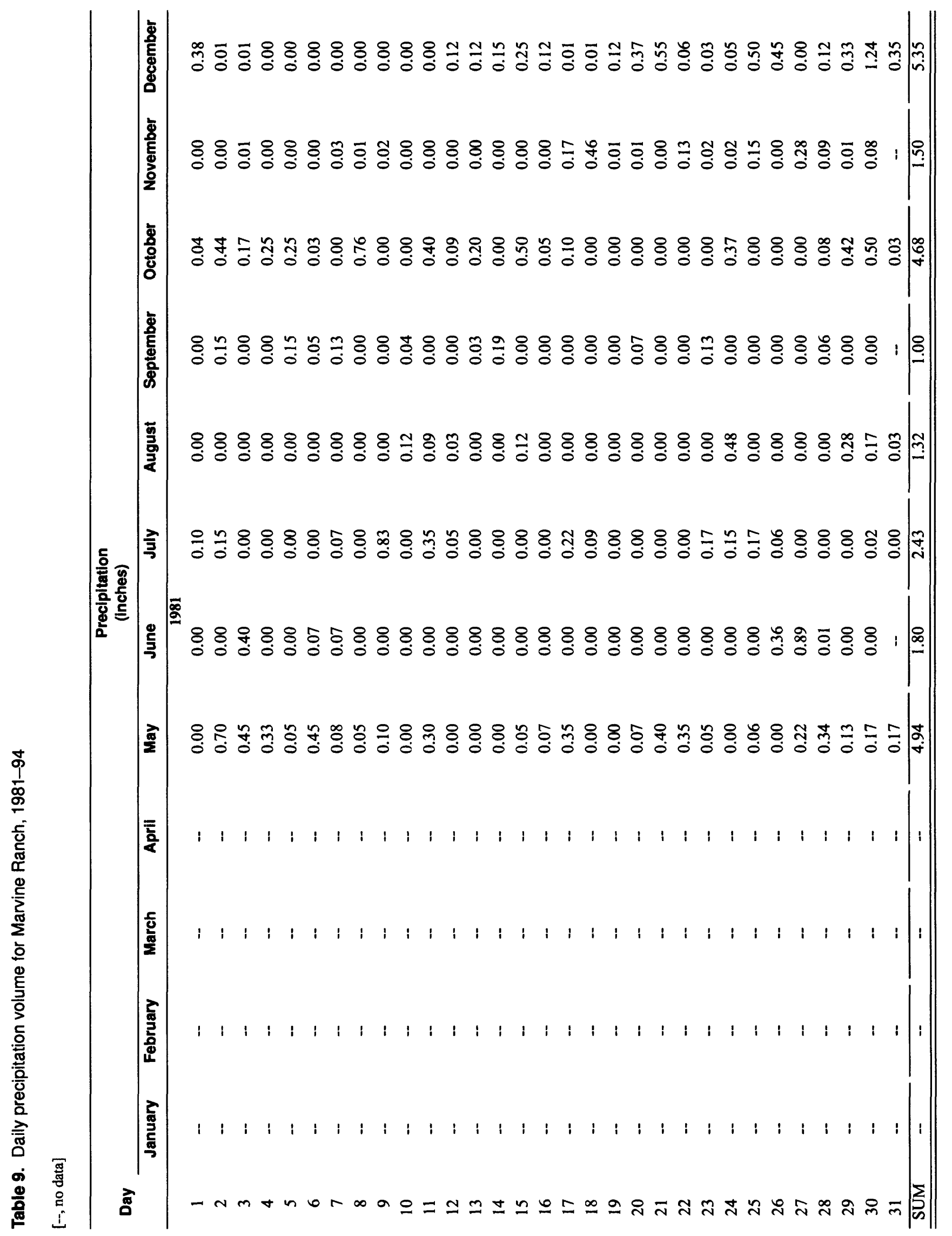




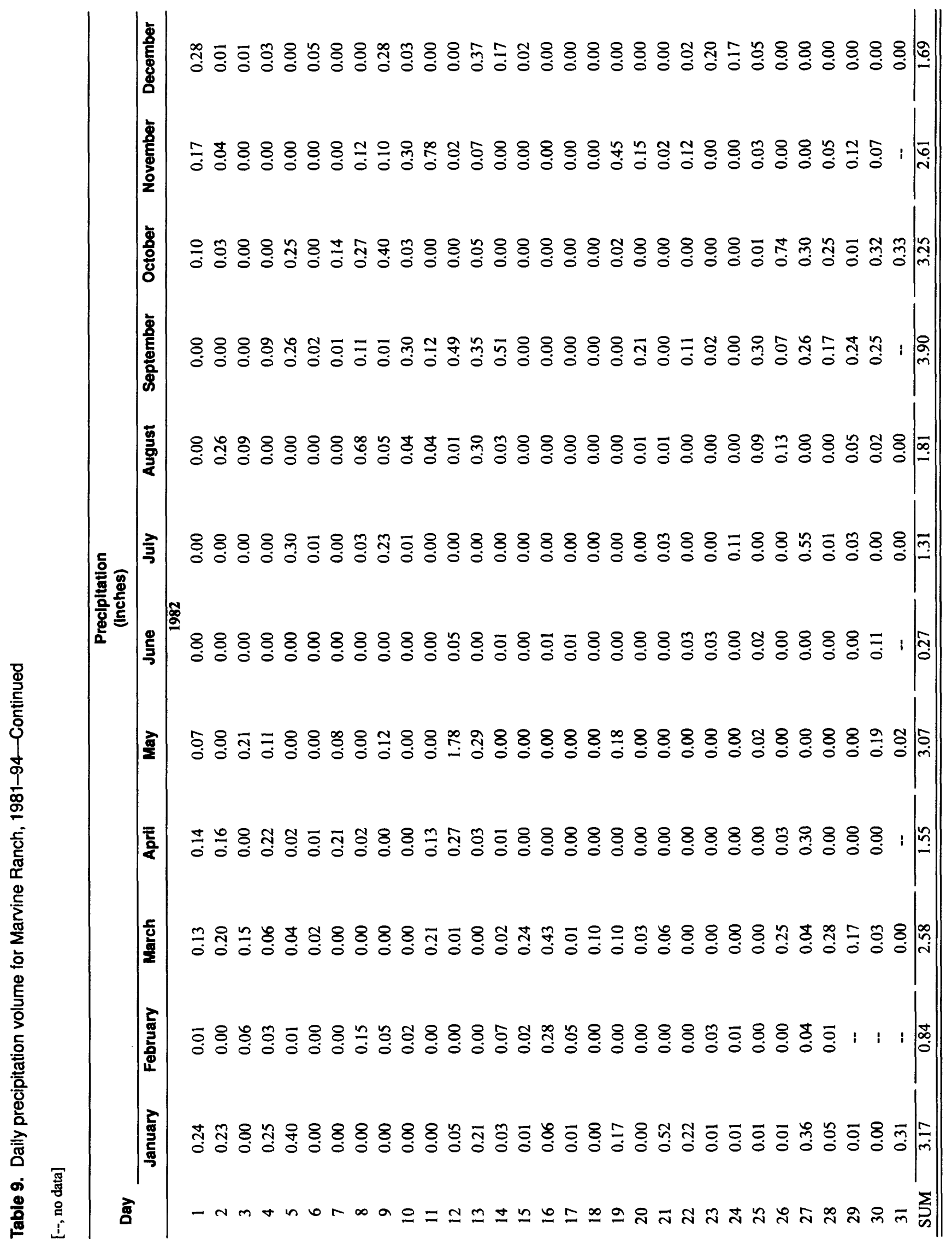




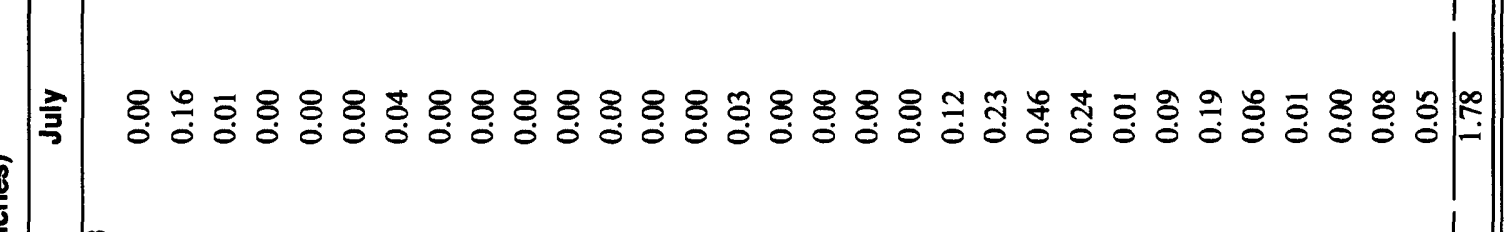
ஓ 8 융 일 そั 둥 8 웅 8 웅 8 웅

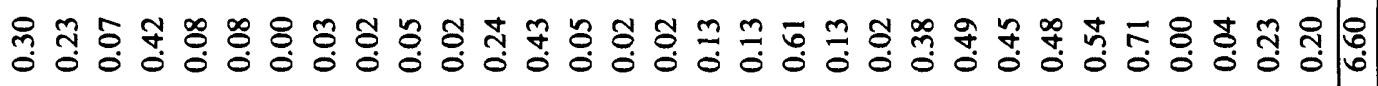

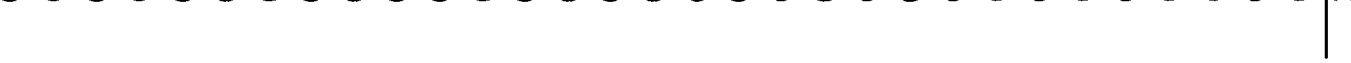

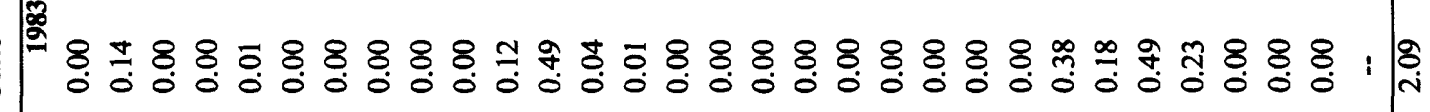
(2)

సิ สุ

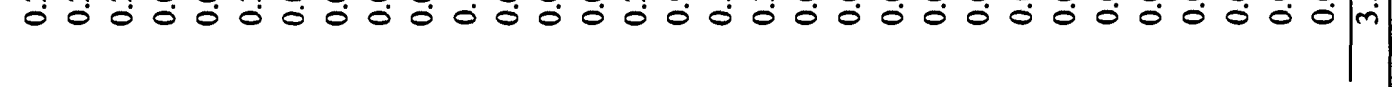

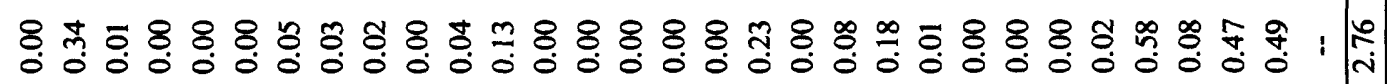

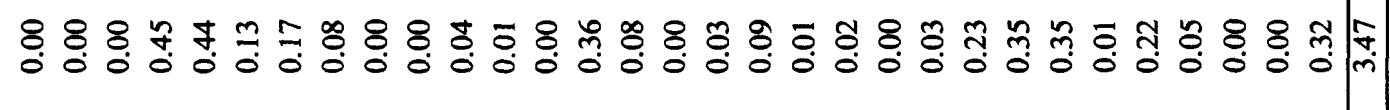

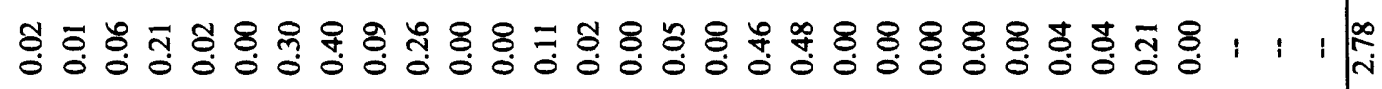

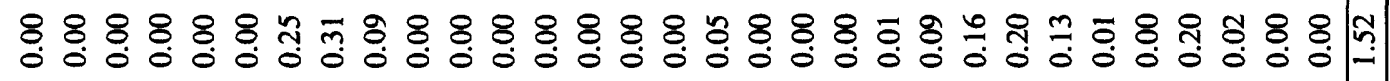




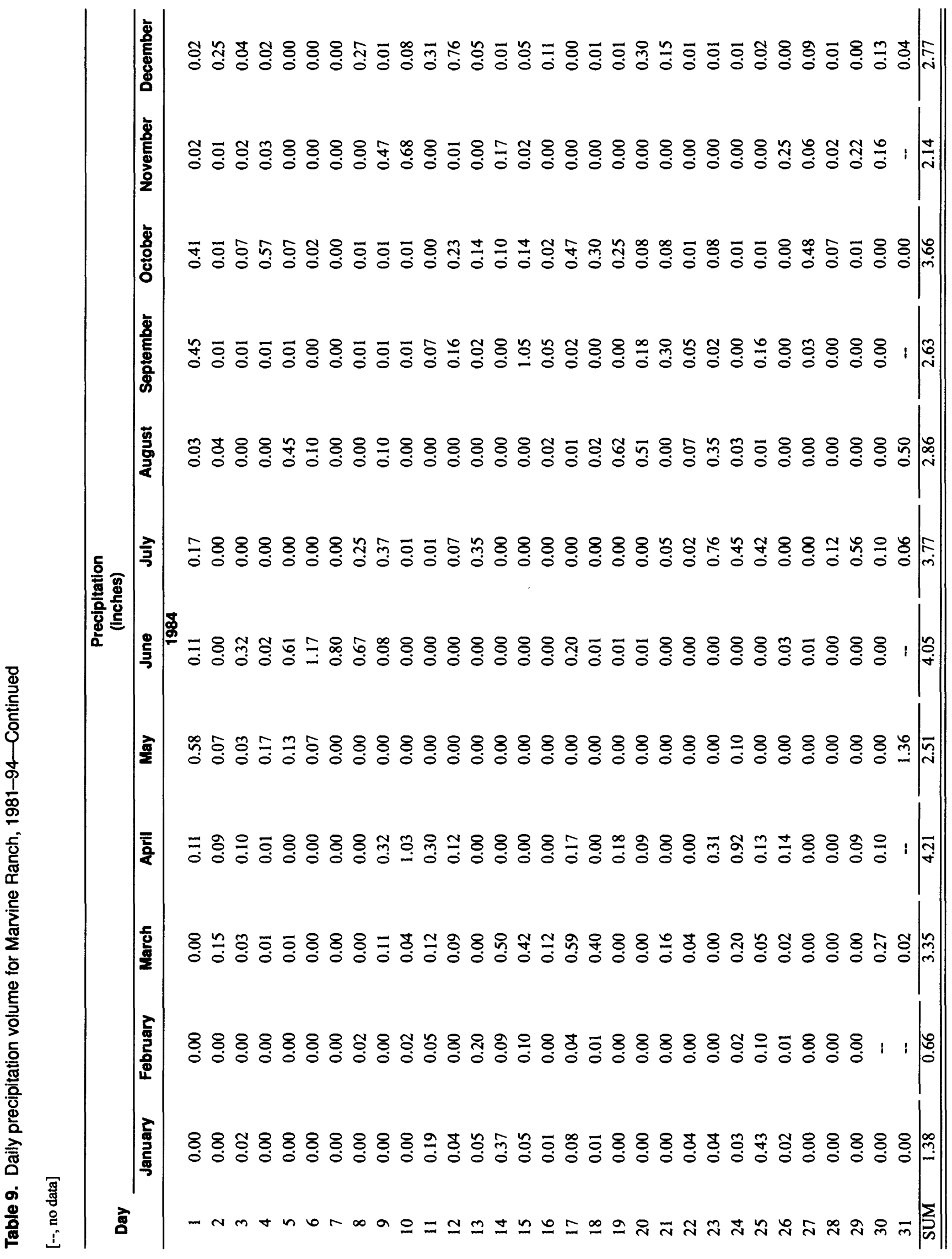




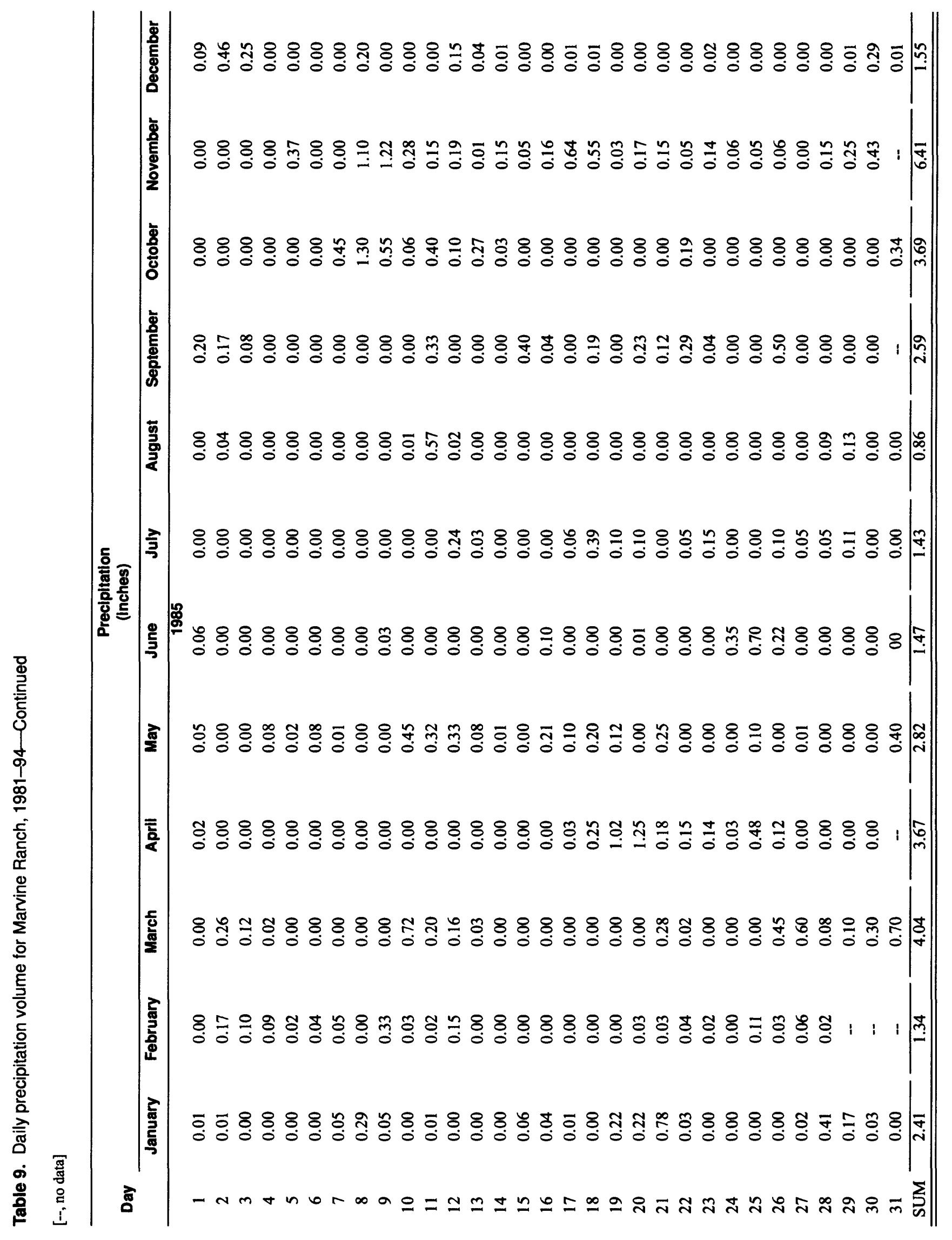




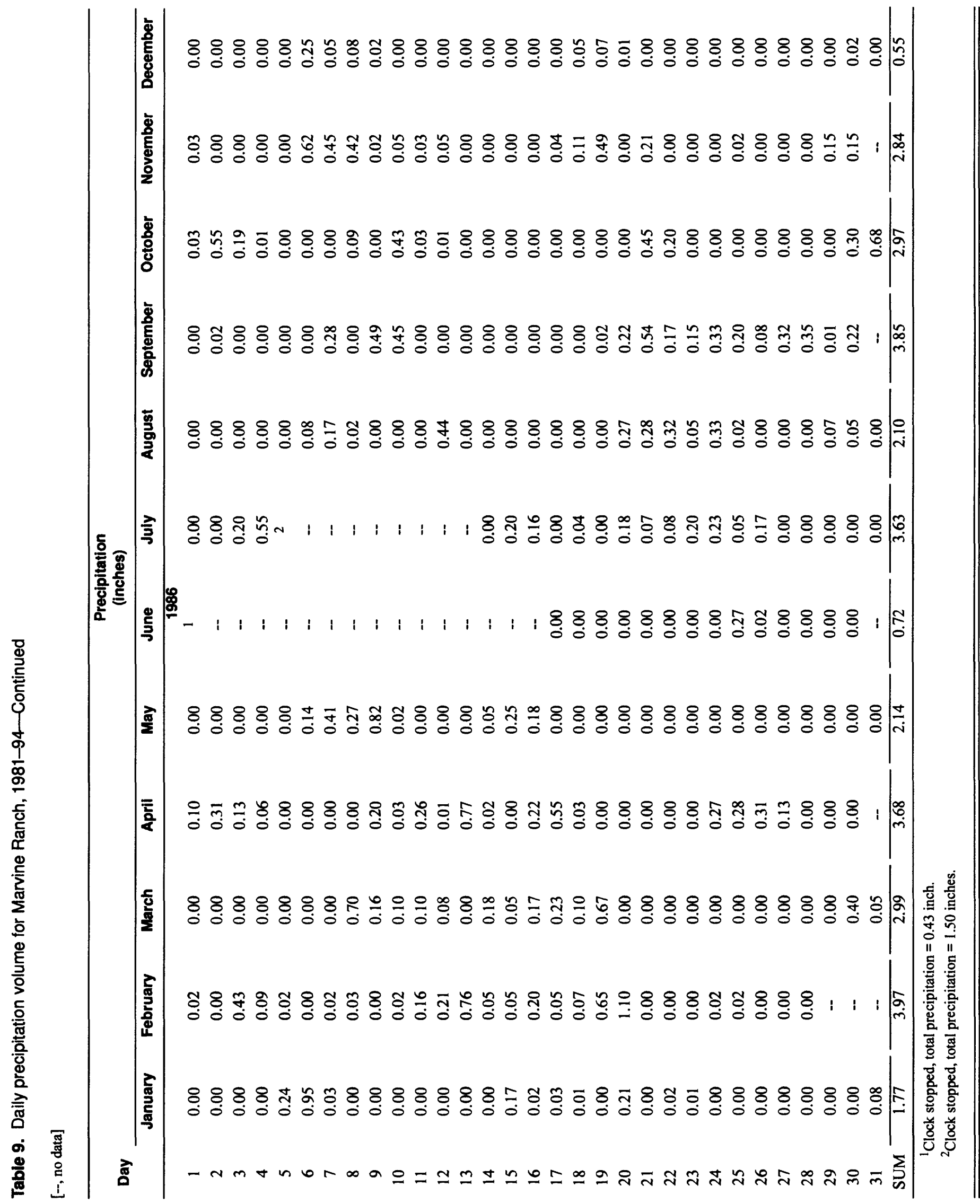




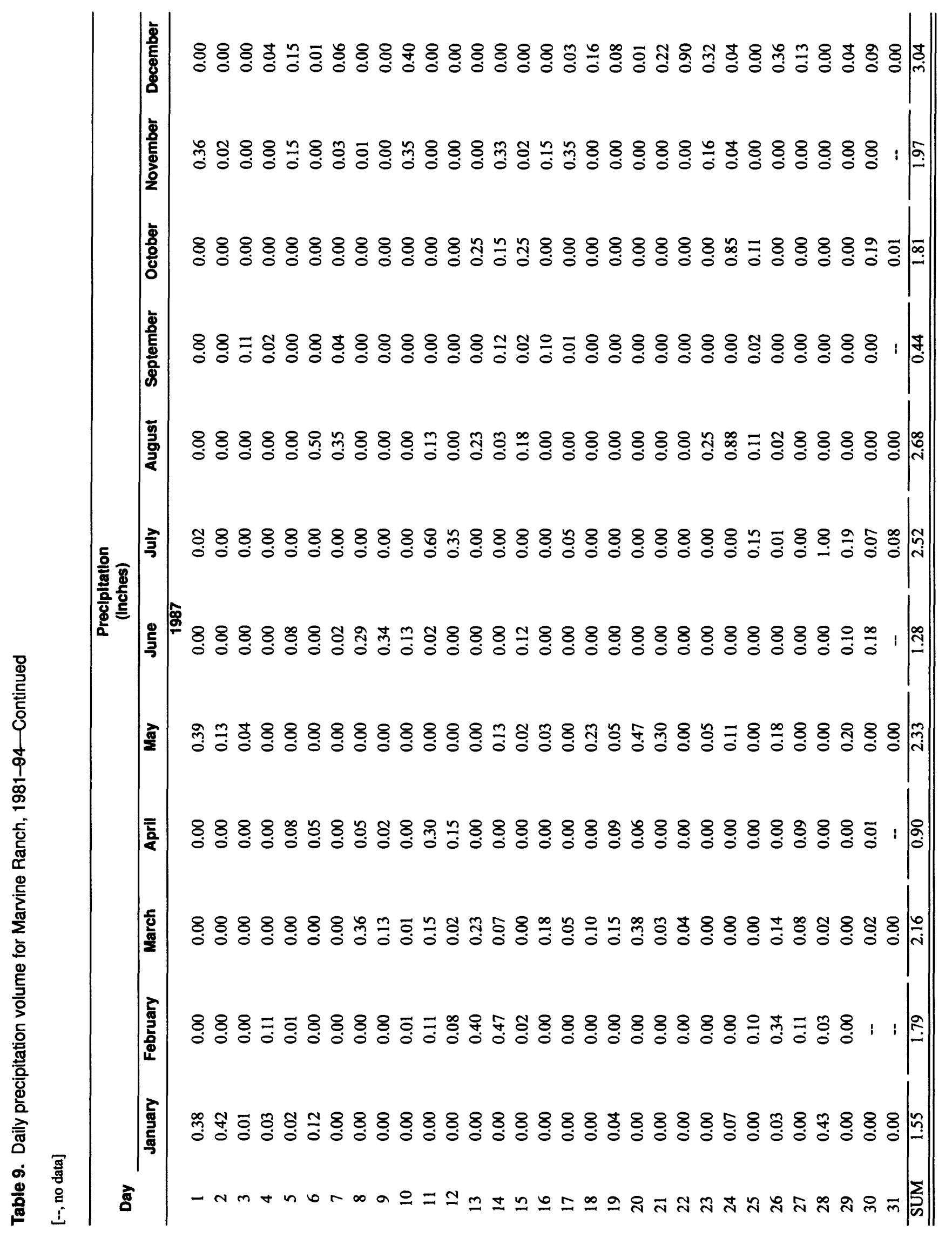




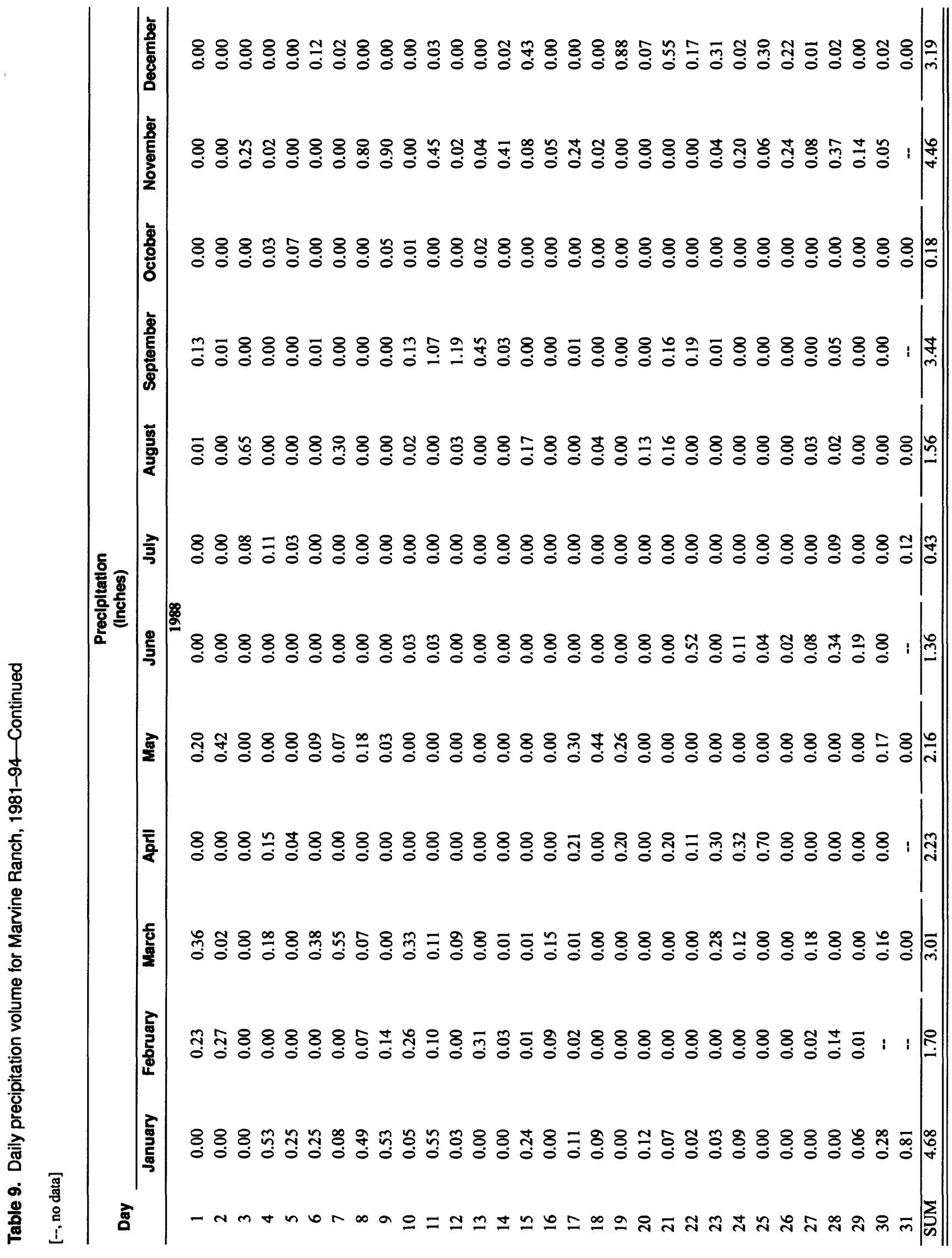




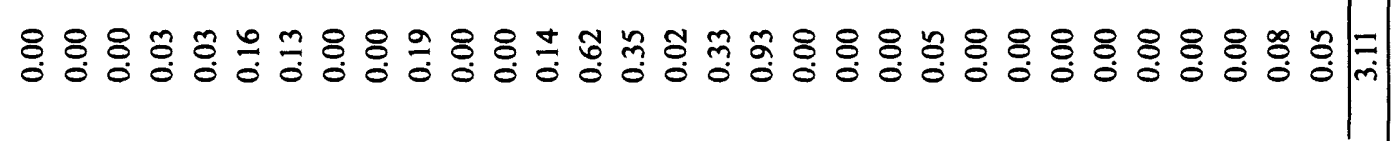

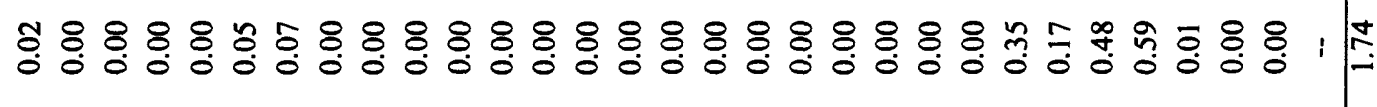

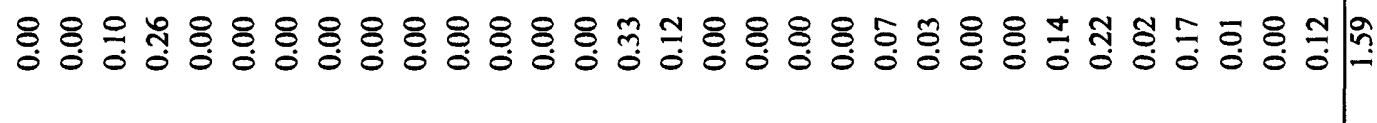

\& \& : \&

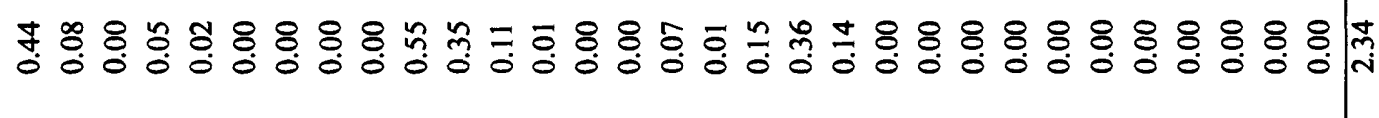

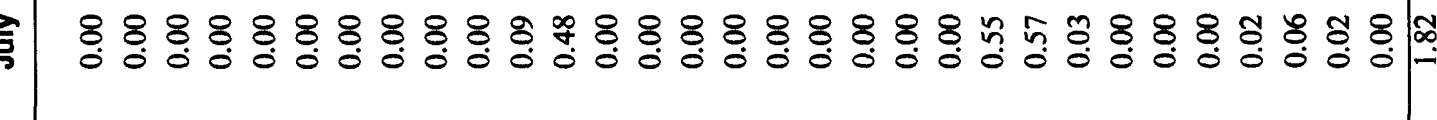

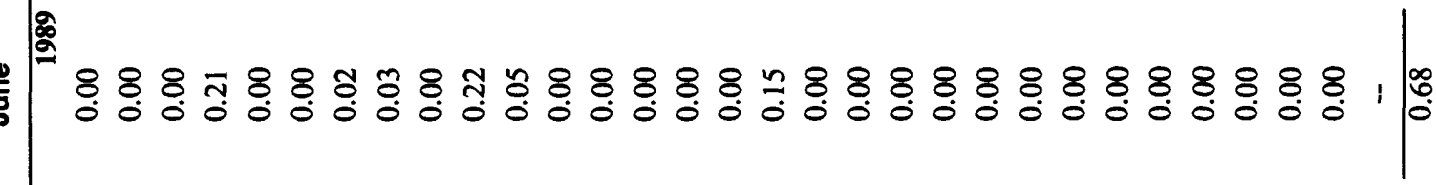

商

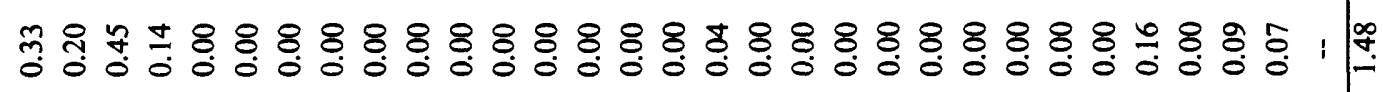
更

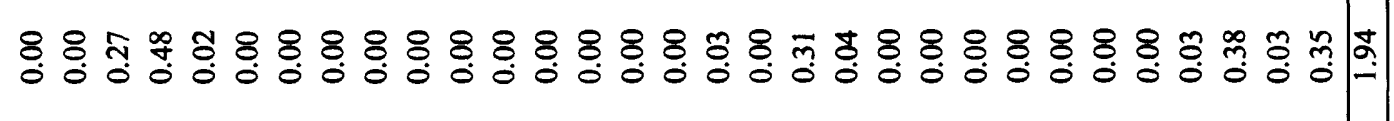

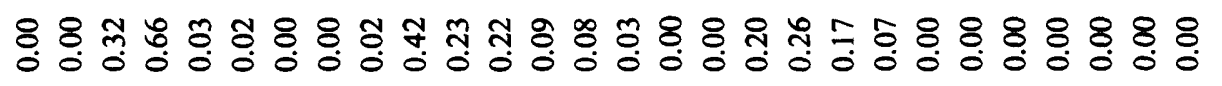

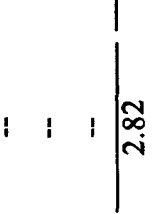

更

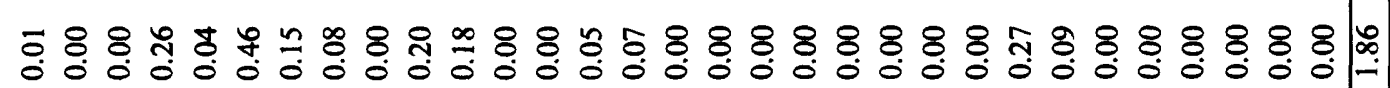




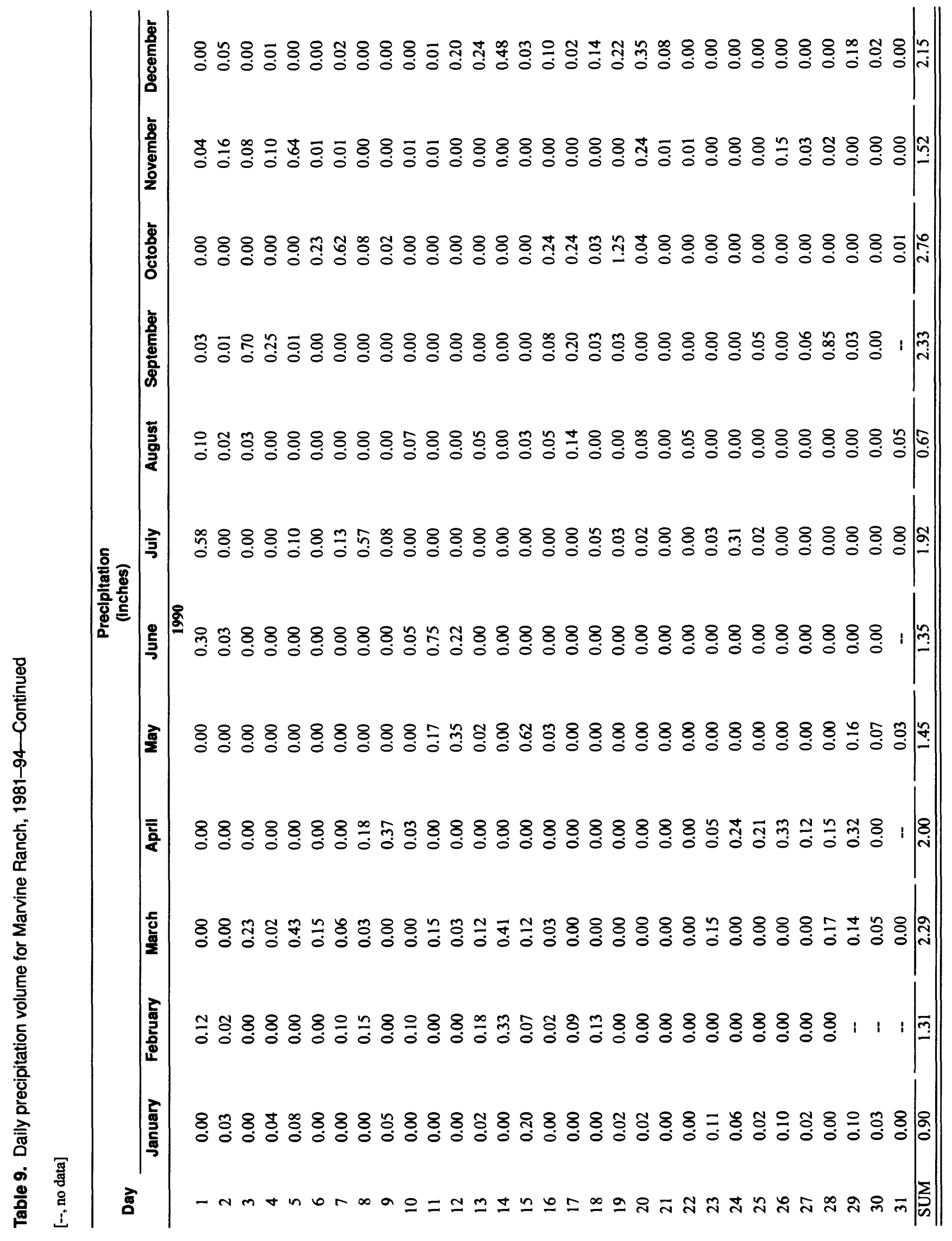




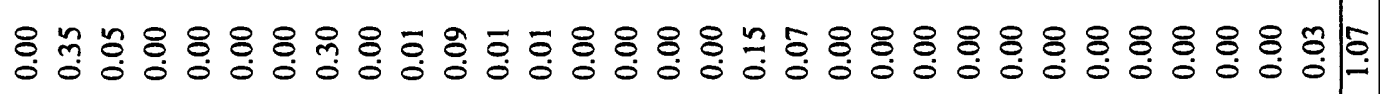

危

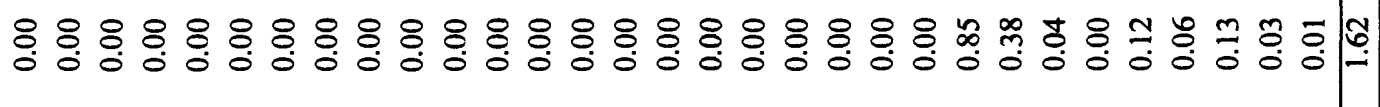

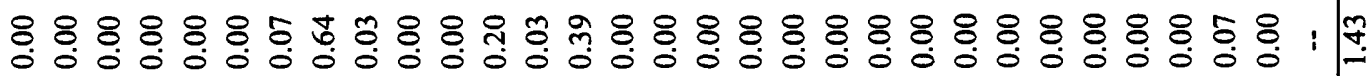

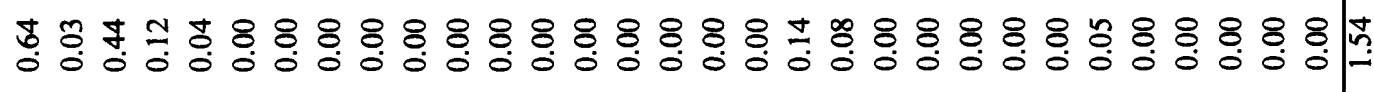

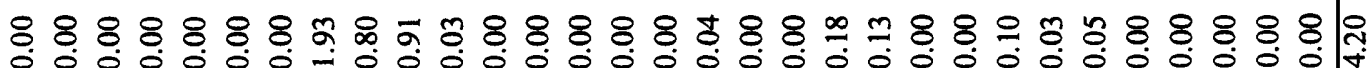

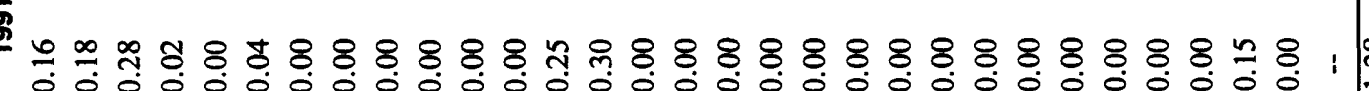
(1)

웅융영

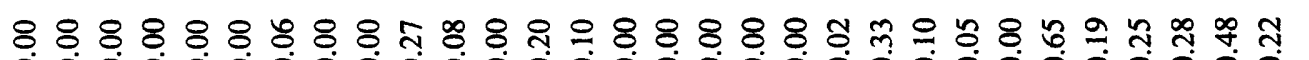

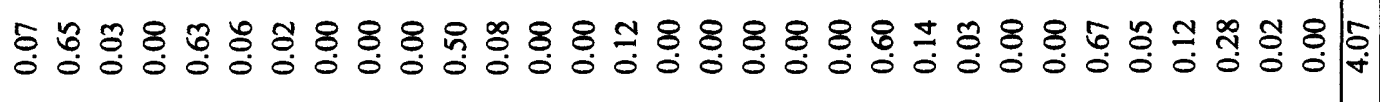

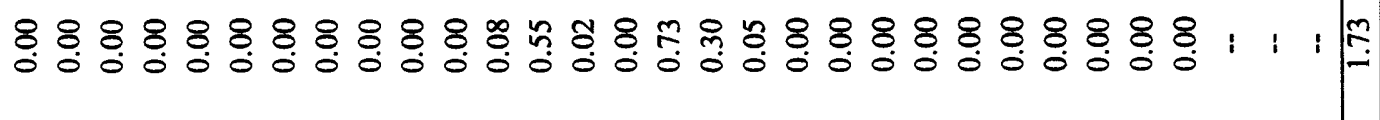

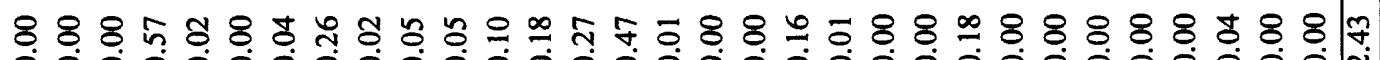

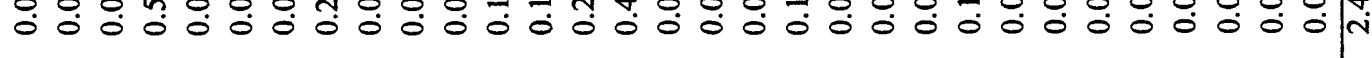




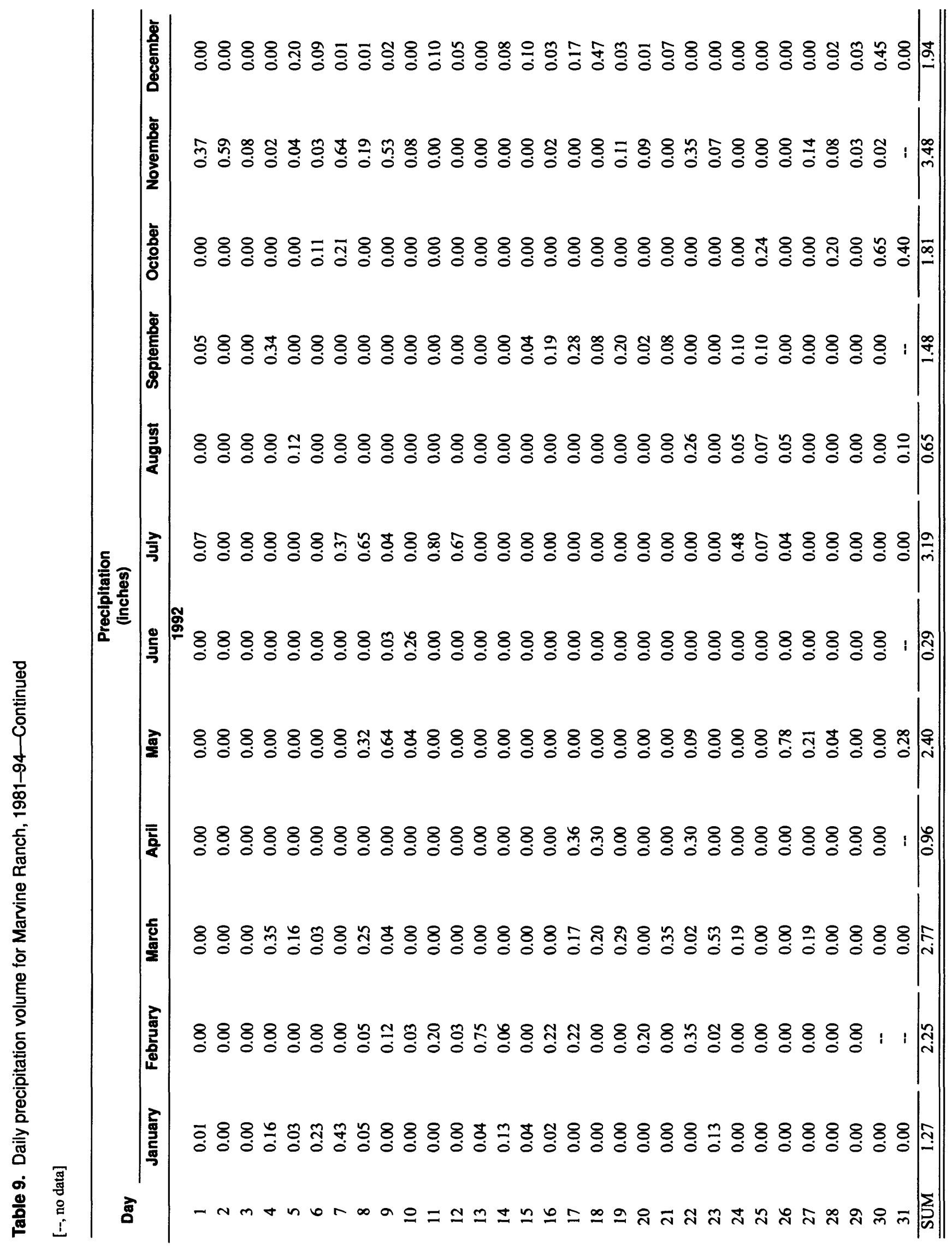




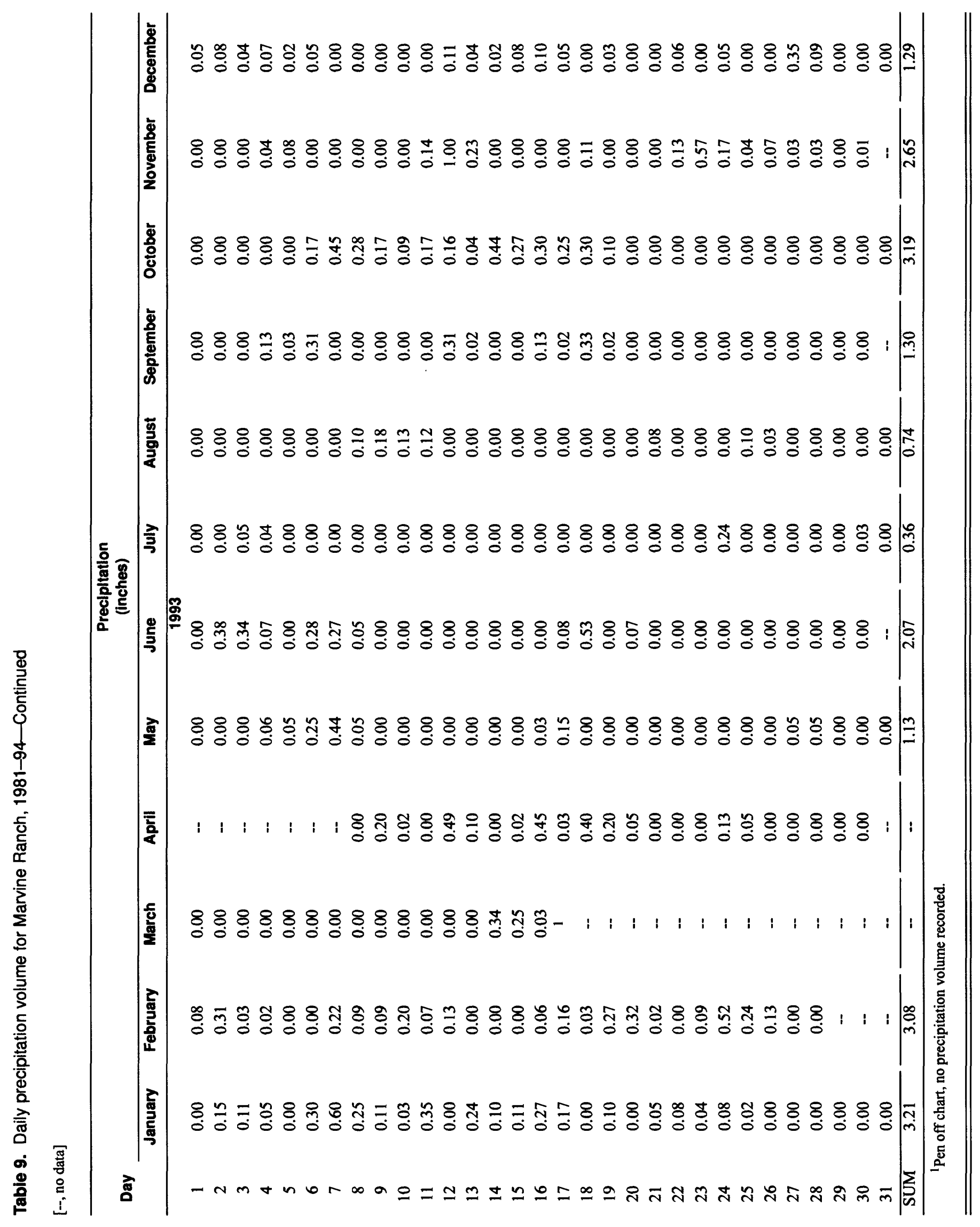




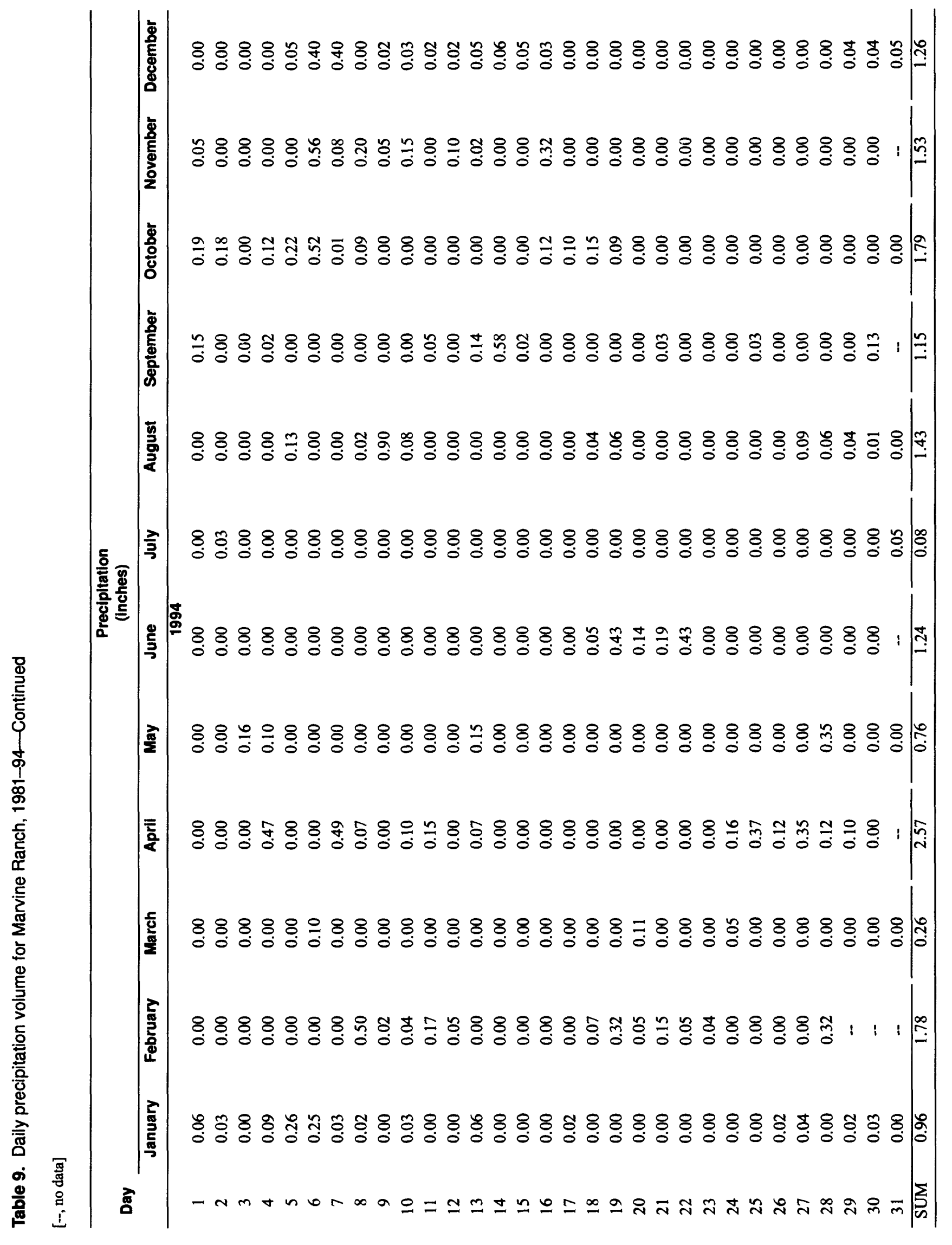

